The Annals of Statistics

2010, Vol. 38, No. 3, 1478-1545

DOI: $10.1214 / 09-A O S 756$

(C) Institute of Mathematical Statistics, 2010

\title{
LIMIT THEOREMS FOR MOVING AVERAGES OF DISCRETIZED PROCESSES PLUS NOISE
}

\author{
By Jean Jacod, Mark Podolskij and Mathias Vetter ${ }^{1}$ \\ UPMC (Université Paris-6), ETH Zürich and Ruhr-Universität Bochum
}

This paper presents some limit theorems for certain functionals of moving averages of semimartingales plus noise which are observed at high frequency. Our method generalizes the pre-averaging approach (see [Bernoulli 15 (2009) 634-658, Stochastic Process. Appl. 119 (2009) 2249-2276]) and provides consistent estimates for various characteristics of general semimartingales. Furthermore, we prove the associated multidimensional (stable) central limit theorems. As expected, we find central limit theorems with a convergence rate $n^{-1 / 4}$, if $n$ is the number of observations.

1. Introduction. The last years have witnessed a considerable development of the statistics of processes observed at very high frequency due to the recent availability of such data. This is particularly the case for market prices of stocks, currencies and other financial instruments. Correlatively, the technology for the analysis of such data has grown rapidly. The emblematic problem is the question of how to estimate daily volatility for financial prices (in stochastic process terms, the quadratic variation of log prices).

However, those high-frequency data are almost always corrupted by some noise. This may be recording or measurement errors, a situation which can be modeled by an additive white noise. For financial data we also have a different sort of "noise" due to the fact that prices are recorded as multiples of the basic currency unit so that some rounding is necessarily performed, and the level of rounding is far from being negligible for very high frequency data in comparison to the intrinsic variability of the underlying process. For these reasons, it is commonly acknowledged that the underlying process of interest, such as the price semimartingale, is latent rather than observed.

Received December 2008; revised June 2009.

${ }^{1}$ Supported by Deutsche Forschungsgemeinschaft through SFB 823.

AMS 2000 subject classifications. Primary 60F05, 60G44, 62M09; secondary 60G42, $62 \mathrm{G} 20$.

Key words and phrases. Central limit theorem, high-frequency observations, microstructure noise, quadratic variation, semimartingale, stable convergence.

This is an electronic reprint of the original article published by the Institute of Mathematical Statistics in The Annals of Statistics,

2010, Vol. 38, No. 3, 1478-1545. This reprint differs from the original in pagination and typographic detail. 
A large amount of work has already been devoted to the subject, especially for additive white noise, but also for some other types of noise like rounding effects. A comprehensive discussion of the noise models and the effect of noise on the inference for the underlying process may be found in [17]. Various statistical procedures for getting rid of the noise have been proposed (see, e.g., $[1,5,9,21,22]$ and, more closely related to the present work, [7, 14, $18,19])$.

Most of the aforementioned papers are concerned with the estimation of the integrated volatility, that is, the quadratic variation, for a continuous semimartingale. Only Podolskij and Vetter [18, 19] deal with estimation of various volatility functionals and robustness to jumps in the discontinuous semimartingale setting with i.i.d. noise. So there is a lack of more general results, allowing, for example, one to estimate other powers of the volatility (like the "quarticity") or the sum of some powers of the jumps, for a general Itô semimartingale. These quantities have proved extremely useful for a number of estimation or testing problems in the context of high-frequency data, but they have been studied when the process is observed without noise. Recall that the typical statistical problems in the noise-free framework are (i) estimation of the quadratic variation (see $[8,13]$ ), (ii) tests for the presence of jumps (see $[4,9]$ ), (iii) tests for the presence of the continuous component (see $[3,10]$ ) or (iv) estimation of the "activity index" of the jump part (see $[2,20])$.

The aim of this paper is to provide probabilistic tools to solve (some of) the aforementioned statistical problems in the presence of noise. Thus this is a rather probabilistic paper, but the interest and motivation of the forthcoming results lie essentially in potential applications; therefore, after the main results we give hints toward how to apply the results for concrete statistical questions, but not a full account of these applications (see, e.g., Remarks 4.2 and 4.5 or Theorem 4.6).

Let us be more specific. We consider an Itô semimartingale $X$ which is corrupted by noise. The observed process $Z=\left(Z_{t}\right)_{t \geq 0}$ is given as

$$
Z_{t}=X_{t}+\chi_{t}, \quad t \geq 0
$$

where $\left(\chi_{t}\right)_{t \geq 0}$ are errors which are, conditionally on the process $X$, centered and independent. The process $Z$ is assumed to be observed at equidistant time points $i \Delta_{n}, i=0,1, \ldots,\left[t / \Delta_{n}\right]$, with $\Delta_{n} \rightarrow 0$ as $n \rightarrow \infty$. This structure of noise allows for an additive white noise but also for noise involving rounding effects since $\chi_{t}$ may depend on $X_{t}$, or even on the whole past of $X$ before time $t$. It rules out, though, some other interesting types of noise, like an additive colored noise. Note, however, that the $\chi_{t}$ are not necessarily independent (the independence is only "conditional on $X$ ").

In the no-noise case (i.e., $\chi \equiv 0$ ) an extensive theory has been developed in various papers which allows for estimating quantities like $\sum_{s \leq t}\left|\Delta X_{s}\right|^{p}$ 
where $\Delta X_{s}$ denotes the jump size of $X$ at time $s$, or $\int_{0}^{t}\left|\sigma_{s}\right|^{p} d s$ where $\sigma$ is the volatility. See, for instance, [6] or [13] among others. Typically, these quantities are estimated by sums of powers of the successive increments of $X$, that is, they are limits of such sums. When noise is present, these estimators are inadequate because they converge toward some characteristics of the noise rather than toward the characteristics of the process $X$ in which we are interested. There are currently three main approaches to overcome this difficulty, mainly for the estimation of the quadratic variation in the continuous case: the subsampling method [21], the realized kernel method [7] and the pre-averaging method [14, 19] (see also [11] for a comprehensive theory in the parametric setting). All these approaches achieve the optimal rate of $\Delta_{n}^{1 / 4}$. In this paper we use the pre-averaging method to derive rather general estimators.

More precisely, we choose a (smooth enough) weight function $g$ on $[0,1]$ and an appropriate sequence $k_{n}$ with which we associate the (observed) variables,

$$
\begin{aligned}
& \bar{Z}(g)_{i}^{n}=\sum_{j=1}^{k_{n}-1} g\left(j / k_{n}\right)\left(Z_{(i+j) \Delta_{n}}-Z_{(i+j-1) \Delta_{n}}\right), \\
& \widehat{Z}(g)_{i}^{n}=\sum_{j=1}^{k_{n}}\left(g\left(j / k_{n}\right)-g\left((j-1) / k_{n}\right)\right)^{2}\left(Z_{(i+j) \Delta_{n}}-Z_{(i+j-1) \Delta_{n}}\right)^{2} .
\end{aligned}
$$

Our aim is to study the asymptotic behavior of the following functionals:

$$
V(Z, g, p, r)_{t}^{n}=\sum_{i=0}^{\left[t / \Delta_{n}\right]-k_{n}}\left|\bar{Z}(g)_{i}^{n}\right|^{p}\left|\widehat{Z}(g)_{i}^{n}\right|^{r}
$$

for suitable powers $p, r \geq 0$. The local smoothing performed by the quantity $\bar{Z}(g)_{i}^{n}$ is somewhat related to the idea proposed in [22] for the estimation of a certain conditional variance. Its role is the reduction of the influence of the noise process $\chi$ whereas $\widehat{Z}(g)_{i}^{n}$ is used for bias corrections. The asymptotic theory for the functionals $V(Z, g, p, 0)_{t}^{n}$ in the absence of jumps is (partially) derived in [14] and [18], but here we extend these results to the case of general semimartingales.

Quite naturally, the asymptotic behavior of $V(Z, g, p, r)_{t}^{n}$ is different according to whether the process $X$ is continuous or not. In particular, different scaling is required to obtain nontrivial limits for $V(Z, g, p, r)_{t}^{n}$. More precisely, we show the following $(\stackrel{\mathbb{P}}{\longrightarrow}$ means convergence in probability, and $\stackrel{\text { u.c.p. }}{\longrightarrow}$ means convergence in probability uniformly over all finite time intervals): 
(i) For all semimartingales $X$ it holds that $\frac{1}{k_{n}} V(Z, g, p, 0)_{t}^{n} \stackrel{\mathbb{P}}{\longrightarrow} \bar{g}(p) \times$ $\sum_{s \leq t}\left|\Delta X_{s}\right|^{p}$ for $p>2$ and $\frac{1}{k_{n}} V(Z, g, 2,0)_{t}^{n}-\frac{1}{2 k_{n}} V(Z, g, 0,1)_{t}^{n} \stackrel{\mathbb{P}}{\longrightarrow} \bar{g}(2)[X, X]_{t}$ where the $\bar{g}(p)$ 's are known constants (which depend on $g$ ), and $[X, X]$ is the quadratic variation of $X$.

(ii) When $X$ is a continuous Itô semimartingale it holds that $\Delta_{n}^{1-p / 4} V(Z$, $g, p, 0)_{t}^{n} \stackrel{\text { u.c.p. }}{\longrightarrow} m_{p} \int_{0}^{t}\left|\theta \bar{g}(2) \sigma_{s}^{2}+\frac{\bar{g}^{\prime}(2)}{\theta} \alpha_{s}^{2}\right|^{p / 2} d s$ where $m_{p}, \theta$ are certain constants, $\left(\sigma_{s}^{2}\right)$ is the volatility process and $\left(\alpha_{s}^{2}\right)$ is the local conditional variance of the noise process $\chi$. Furthermore, a proper linear combination of $V(Z, g, p, r)_{t}^{n}$ for integers $p, r$ with $p+2 r=l$ converges in probability to $\int_{0}^{t}\left|\sigma_{s}\right|^{l} d s$ when $l$ is an even integer.

For each of the aforementioned cases we prove a joint stable central limit theorem for a given family of weight functions $\left(g_{i}\right)_{1 \leq i \leq d}$ [for the first functional in (i) we additionally have to assume that $p>3]$. The corresponding convergence rate is $\Delta_{n}^{1 / 4}$.

We end this introduction by emphasizing that only the one-dimensional case for $X$ is studied here. The extension to multi-dimensional semimartingales is possible, and even mathematically rather straightforward, but extremely cumbersome.

This paper is organized as follows: in Section 2 we introduce the setting and the assumptions. Sections 3 and 4 are devoted to stating the results, first the various convergences in probability and second the associated central limit theorems. The proofs are gathered in Section 5 .

2. The setting. We have a one-dimensional underlying process $X=\left(X_{t}\right)_{t \geq 0}$, and observation times $i \Delta_{n}$ for all $i=0,1, \ldots, k, \ldots$ with $\Delta_{n} \rightarrow 0$. We suppose that $X$ is a semimartingale which can thus be written as

$$
X=X_{0}+B+X^{c}+\left(x 1_{\{|x| \leq 1\}}\right) \star(\mu-\nu)+\left(x 1_{\{|x|>1\}}\right) \star \mu .
$$

Here $\mu$ is the jump measure of $X$ with $\nu$ its predictable compensator; $X^{c}$ is the continuous (local) martingale part of $X$, and $B$ is the drift. All these are defined on some filtered probability space $\left(\Omega^{(0)}, \mathcal{F}^{(0)},\left(\mathcal{F}_{t}^{(0)}\right)_{t \geq 0}, \mathbb{P}^{(0)}\right)$. We use here the usual notation of stochastic calculus, and for any unexplained (but standard) notation we refer to [16]; for example $\psi \star(\mu-\nu)_{t}=$ $\int_{0}^{t} \int_{\mathbb{R}} \psi(s, x)(\mu-\nu)(d s, d x)$ is the stochastic integral of the predictable function $\psi(\omega, t, x)$ with respect to the martingale measure $\mu-\nu$, when it exists.

The process $X$ is observed with an error; that is, at stage $n$, and instead of the values $X_{i}^{n}=X_{i \Delta_{n}}$ for $i \geq 0$, we observe $X_{i}^{n}+\chi_{i}^{n}$ where the $\chi_{i}^{n}$ 's are "errors" which are, conditionally on the process $X$, centered and independent (this allows for errors which are depending on $X$ and thus may be unconditionally dependent). It is convenient to define the noise $\chi_{t}$ for any time $t$, although at stage $n$ only the values $\chi_{i \Delta_{n}}$ are really used. 
Mathematically speaking, this can be formalized as such: for each $t \geq 0$, we have a transition probability $Q_{t}\left(\omega^{(0)}, d z\right)$ from $\left(\Omega^{(0)}, \mathcal{F}_{t}^{(0)}\right)$ into $\mathbb{R}$. We endow the space $\Omega^{(1)}=\mathbb{R}^{[0, \infty)}$ with the product Borel $\sigma$-field $\mathcal{F}^{(1)}$ and the "canonical process" $\left(\chi_{t}: t \geq 0\right)$ and with the probability $\mathbb{Q}\left(\omega^{(0)}, d \omega^{(1)}\right)$ which is the product $\bigotimes_{t>0} Q_{t}\left(\omega^{(0)}, \cdot\right)$. We introduce the filtered probability space $\left(\Omega, \mathcal{F},\left(\mathcal{F}_{t}\right)_{t \geq 0}, \mathbb{P}\right)$ and the filtration $\left(\mathcal{G}_{t}\right)$ as follows:

$$
\begin{aligned}
& \Omega=\Omega^{(0)} \times \Omega^{(1)}, \quad \mathcal{F}=\mathcal{F}^{(0)} \otimes \mathcal{F}^{(1)}, \\
& \left.\mathcal{F}_{t}=\mathcal{F}_{t}^{(0)} \otimes \sigma\left(\chi_{s}: s \in[0, t)\right), \quad \mathcal{G}_{t}=\mathcal{F}^{(0)} \otimes \sigma\left(\chi_{s}: s \in[0, t)\right),\right\} \\
& \mathbb{P}\left(d \omega^{(0)}, d \omega^{(1)}\right)=\mathbb{P}^{(0)}\left(d \omega^{(0)}\right) \mathbb{Q}\left(\omega^{(0)}, d \omega^{(1)}\right) .
\end{aligned}
$$

Any variable or process which is defined on either $\Omega^{(0)}$ or $\Omega^{(1)}$ can be considered in the usual way as a variable or a process on $\Omega$. Note that $X$ is still a semimartingale with the same decomposition $(2.1)$ on $\left(\Omega, \mathcal{F},\left(\mathcal{F}_{t}\right)_{t>0}, \mathbb{P}\right)$ despite the fact that the filtration $\left(\mathcal{F}_{t}\right)$ is not right-continuous. On the other hand, the "process" $\chi$ typically has no measurable property in time since under $\mathbb{Q}\left(\omega^{(0)}, \cdot\right)$ it is constituted of independent variables; as mentioned before, only the values of $\chi$ at the observation times are relevant, and the extension as a process indexed by $\mathbb{R}_{+}$is for notational convenience only.

At time $t$, instead of $X_{t}$, we observe the variable

$$
Z_{t}=X_{t}+\chi_{t}
$$

We make the following crucial assumption on the noise, for some $q \geq 2$ :

Hypothesis $(N-q)$. There is a sequence of $\left(\mathcal{F}_{t}^{(0)}\right)$-stopping times $\left(T_{n}\right)$ increasing to $\infty$, such that $\int Q_{t}\left(\omega^{(0)}, d z\right)|z|^{q} \leq n$ whenever $t<T_{n}\left(\omega^{(0)}\right)$. We write for any integer $r \leq q$,

$$
\beta(r)_{t}\left(\omega^{(0)}\right)=\int Q_{t}\left(\omega^{(0)}, d z\right) z^{r}, \quad \alpha_{t}=\sqrt{\beta(2)_{t}},
$$

and we also assume that

$$
\beta(1) \equiv 0 .
$$

In most applications, the local boundedness of the $q$ th moment of the noise, even for all $q>0$, is not a serious restriction. Condition (2.5), on the other hand, is a quite serious restriction (see [14] for a discussion of the implications of this assumption, and below are some examples).

EXAMPLE 2.1. The structure of the noise allows for an additive white noise [all $Q_{t}\left(\omega^{(0)}, \cdot\right)$ are equal to a fixed probability measure, independent of $\left(\omega^{(0)}, t\right)$, with mean 0]. It also allows for some sort of rounding which means that the observed process $Z_{t}$ takes its values in $\alpha \mathbb{Z}$ where $\alpha>0$ is the rounding level; for example if the $\xi_{t}$ are i.i.d. uniform on $[0, \alpha]$ and independent 
of $\mathcal{F}^{(0)}$ (hence of $X$ ) and $Z_{t}=\alpha\left[\left(X_{t}+\xi_{t}\right) / \alpha\right]$ (here $[x]$ denotes the integer part of the real $x)$, we have $Q_{t}(\cdot, d x)=\left(X_{t} / \alpha-\left[X_{t} / \alpha\right]\right) \varepsilon_{\alpha\left[X_{t} / \alpha\right]+1-X_{t}}(d x)+$ $\left(1-X_{t} / \alpha+\left[X_{t} / \alpha\right]\right) \varepsilon_{\alpha\left[X_{t} / \alpha\right]-X_{t}}(d x)$ which satisfies Hypothesis $(N-q)$ for all $q$ (here $\varepsilon$ denotes the Dirac measure). Many other specifications of rounding errors are possible, obviously.

However, it unfortunately does not allow for "pure rounding," that is, $Z_{t}=\alpha\left[X_{t} / \alpha\right]$; although in this case we have the structure (2.2), the property (2.5) is violated. In this case, there is no way of estimating the integrated volatility in a consistent way because this quantity is not even a function of the path $t \mapsto Z_{t}$ in the "completely observed" case.

We choose a sequence of integers $k_{n}$ satisfying for some $\theta>0$,

$$
k_{n} \sqrt{\Delta_{n}}=\theta+\mathrm{o}\left(\Delta_{n}^{1 / 4}\right) ; \quad \text { we write } u_{n}=k_{n} \Delta_{n} .
$$

We will also consider weight functions $g$ on $[0,1]$, satisfying

$\left.\begin{array}{l}g \text { is continuous, piecewise } C^{1} \text { with a piecewise Lipschitz derivative } g^{\prime}, \\ g(0)=g(1)=0, \quad \int_{0}^{1} g(s)^{2} d s>0 .\end{array}\right\}$

It is convenient to extend such a $g$ to the whole of $\mathbb{R}$ by setting $g(s)=0$ if $s \notin[0,1]$. We associate with $g$ the following numbers [where $p \in(0, \infty)$ and $i \in \mathbb{Z}]$ :

$$
\left.\begin{array}{l}
g_{i}^{n}=g\left(i / k_{n}\right), \quad g_{i}^{\prime n}=g_{i}^{n}-g_{i-1}^{n}, \\
\bar{g}(p)_{n}=\sum_{i=1}^{k_{n}}\left|g_{i}^{n}\right|^{p}, \quad \bar{g}^{\prime}(p)_{n}=\sum_{i=1}^{k_{n}}\left|g_{i}^{\prime n}\right|^{p} \cdot
\end{array}\right\}
$$

If $g, h$ are bounded functions with support in $[0,1]$, and $p>0$ and $t \in \mathbb{R}$, we set

$$
\bar{g}(p)=\int|g(s)|^{p} d s, \quad \overline{(g h)}(t)=\int g(s) h(s-t) d s .
$$

For example, $\bar{g}^{\prime}(p)$ is associated with $g^{\prime}$ by the first definition above, and $\bar{g}(2)=\overline{(g g)}(0)$. Note that, as $n \rightarrow \infty$,

$$
\bar{g}(p)_{n}=k_{n} \bar{g}(p)+\mathrm{O}(1), \quad \bar{g}^{\prime}(p)_{n}=k_{n}^{1-p} \bar{g}^{\prime}(p)+\mathrm{O}\left(k_{n}^{-p}\right) .
$$

With any process $Y=\left(Y_{t}\right)_{t \geq 0}$ we associate the following random variables:

$$
\left.\begin{array}{l}
Y_{i}^{n}=Y_{i \Delta_{n}}, \quad \Delta_{i}^{n} Y=Y_{i \Delta_{n}}-Y_{(i-1) \Delta_{n}}, \\
\bar{Y}(g)_{i}^{n}=\sum_{j=1}^{k_{n}-1} g_{j}^{n} \Delta_{i+j}^{n} Y=-\sum_{j=1}^{k_{n}} g_{j}^{\prime n} Y_{i+j-1}^{n}, \\
\widehat{Y}(g)_{i}^{n}=\sum_{j=1}^{k_{n}}\left(g_{j}^{\prime n} \Delta_{i+j}^{n} Y\right)^{2},
\end{array}\right\}
$$


and we define the $\sigma$-fields $\mathcal{F}_{i}^{n}=\mathcal{F}_{i \Delta_{n}}$ and $\mathcal{G}_{i}^{n}=\mathcal{G}_{i \Delta_{n}}$.

Now we can define the processes of interest for this paper. Below, $p$ and $r$ are nonnegative reals, and typically the process $Y$ will be $X$ or $Z$.

$$
V(Y, g, p, r)_{t}^{n}=\sum_{i=0}^{\left[t / \Delta_{n}\right]-k_{n}}\left|\bar{Y}(g)_{i}^{n}\right|^{p}\left|\widehat{Y}(g)_{i}^{n}\right|^{r} .
$$

REMARK 2.2. The process $V(Z, g, p, 0)_{t}^{n}$ is the realized $p$-variation of moving averages of the observations $Z_{i \Delta_{n}}$ over a window of size $u_{n}=k_{n} \Delta_{n}$ and is designed to wipe out the influence of the noise. The influence of the noise after using this procedure is of order of magnitude $1 / \sqrt{k_{n}}$ because the averaging uses $k_{n}$ observations. On the other hand when there is no noise but we still take moving averages, the rate of convergence of our functionals are typically $\sqrt{u_{n}}$ because at time $t$ the summands (the number of which is about $t / \Delta_{n}$ ) are strongly dependent; if we want enough independence to obtain a CLT we basically have to consider nonoverlapping intervals whose number is about $t / u_{n}$.

The "overall" rate of convergence is of order $\frac{1}{\sqrt{k_{n}}} \vee \sqrt{u_{n}}$; this explains the choice (2.6) for $k_{n}$ which amounts to optimizing the rate. Of course, doing so does not completely wipe out the noise which then comes as a bias; this is why we need the complicated processes $V(Z, g, p, r)_{t}^{n}$ in order to remove this bias (see Remark 2.3 below).

Finally we state our assumptions on $X$. One of these is that $X$ is an Ito semimartingale. This means that its characteristics are absolutely continuous with respect to Lebesgue measure, or equivalently that it can be written as

$$
\begin{aligned}
X_{t}= & X_{0}+\int_{0}^{t} b_{s} d s+\int_{0}^{t} \sigma_{s} d W_{s} \\
& +\left(\delta 1_{\{|\delta| \leq 1\}}\right) \star(\underline{\mu}-\underline{\nu})_{t}+\left(\delta 1_{\{|\delta|>1\}}\right) \star \underline{\mu}_{t},
\end{aligned}
$$

where $W$ is a Brownian motion and $\mu$ and $\underline{\nu}$ are a Poisson random measure on $\mathbb{R}_{+} \times E$, and its compensator $\underline{\nu}(d t, d z)=d t \otimes \lambda(d z)$ [where $(E, \mathcal{E})$ is an auxiliary space and $\lambda$ a $\sigma$-finite measure]. The required regularity and boundedness conditions on the coefficients $b, \sigma, \delta$ are gathered in the following:

Hypothesis $(\mathrm{H})$. The process $X$ has the form (2.13) [on $\left(\Omega^{(0)}, \mathcal{F}^{(0)}\right.$, $\left.\left.\left(\mathcal{F}_{t}^{(0)}\right), \mathbb{P}^{(0)}\right)\right]$, and:

(a) the process $\left(b_{t}\right)$ is optional and locally bounded; 
(b) the processes $\left(\sigma_{t}\right)$ is càdlàg (= right-continuous with left limits) and adapted;

(c) the function $\delta$ is predictable, and there is a bounded function $\gamma$ in $\mathbb{L}^{2}(E, \mathcal{E}, \lambda)$ such that the process $\sup _{z \in E}\left(\left|\delta\left(\omega^{(0)}, t, z\right)\right| \wedge 1\right) / \gamma(z)$ is locally bounded.

In particular, a continuous Itô semimartingale is of the form

$$
X_{t}=X_{0}+\int_{0}^{t} b_{s} d s+\int_{0}^{t} \sigma_{s} d W_{s}
$$

where the processes $b$ and $\sigma$ are optional [relative to $\left(\mathcal{F}_{t}^{(0)}\right)$ ] and such that the integrals above make sense. When this is the case, we sometimes need the process $\sigma$ itself to be an Itô semimartingale; it can then be written as in (2.13), but another way of expressing this property is as follows [we are again on the space $\left.\left(\Omega^{(0)}, \mathcal{F}^{(0)},\left(\mathcal{F}_{t}^{(0)}\right), \mathbb{P}^{(0)}\right)\right]$ :

$$
\sigma_{t}=\sigma_{0}+\int_{0}^{t} \widetilde{b}_{s} d s+\int_{0}^{t} \widetilde{\sigma}_{s} d W_{s}+M_{t}+\sum_{s \leq t} \Delta \sigma_{s} 1_{\left\{\left|\Delta \sigma_{s}\right|>v\right\}}
$$

where $M$ is a local martingale orthogonal to $W$ and with bounded jumps and $\langle M, M\rangle_{t}=\int_{0}^{t} a_{s} d s$, and the compensator of $\sum_{s \leq t} 1_{\left\{\left|\Delta \sigma_{s}\right|>v\right\}}$ is $\int_{0}^{t} a_{s}^{\prime} d s$, and where $\widetilde{b}_{t}, a_{t}, a_{t}^{\prime}$ and $\widetilde{\sigma}_{t}$ are optional processes; the first three being locally integrable and the fourth being locally square-integrable. Then we set the following:

Hypothesis (K). We have (2.14) and (2.15), and the processes $\widetilde{b}_{t}, a_{t}$, $a_{t}^{\prime}$ are locally bounded whereas the processes $b_{t}$ and $\widetilde{\sigma}_{t}$ are left-continuous with right limits.

REMARK 2.3. (i) The intuition behind the quantities $\bar{Z}(g)_{i}^{n}$ and $\widehat{Z}(g)_{i}^{n}$ can be explained as follows. Assume for simplicity that $X$ is the continuous Itô semimartingale (2.14) and the noise process $\chi$ is independent of $X$. Now, conditionally on $\mathcal{F}_{i}^{n}$, it holds that

$$
\Delta_{n}^{-1 / 4} \bar{Z}(g)_{i}^{n} \stackrel{\text { asy }}{\sim} \mathcal{N}\left(0, \theta \bar{g}(2) \sigma_{i \Delta_{n}}^{2}+\frac{\bar{g}^{\prime}(2)}{\theta} \alpha_{i \Delta_{n}}^{2}\right)
$$

when the processes $\alpha$ and $\sigma$ are continuous on the interval $\left(i \Delta_{n},\left(i+k_{n}\right) \Delta_{n}\right]$. On the other hand, we have that

$$
\widehat{Z}(g)_{i}^{n} \approx \frac{2 \bar{g}^{\prime}(2)}{k_{n}} \alpha_{i \Delta_{n}}^{2},
$$

when the process $\alpha$ is continuous on the interval $\left(i \Delta_{n},\left(i+k_{n}\right) \Delta_{n}\right.$ ] (this approximation holds even for all semimartingales $X)$. It is now intuitively 
clear that a certain combination of the quantities $\bar{Z}(g)_{i}^{n}$ and $\widehat{Z}(g)_{i}^{n}$ can be used to estimate some functions of $\sigma_{i \Delta_{n}}$ (which is usually the main object of interest). In particular, a proper linear combination of $V(Y, g, p-2 l, l)_{t}^{n}$, $l=0, \ldots, p / 2$, for an even number $p$, converges in probability to $\int_{0}^{t}\left|\sigma_{s}\right|^{p} d s$. This intuition is formalized in Theorems 3.3 and 3.4.

(ii) In the continuous case the quantities $\bar{Z}(g)_{i}^{n}$ and $\widehat{Z}(g)_{i}^{n}$ are asymptotically $k_{n}$-dependent, that is, $\bar{Z}(g)_{i}^{n}\left[\right.$ resp., $\left.\widehat{Z}(g)_{i}^{n}\right]$ is asymptotically (conditionally) independent of $\bar{Z}(g)_{j}^{n}\left[\right.$ resp., $\left.\widehat{Z}(g)_{j}^{n}\right]$ when $|i-j|>k_{n}$. Thus we will apply a classical block splitting technique for $m$-dependent variables to derive the central limit theorem for $V(Y, g, p, r)_{t}^{n}$ when $X$ is continuous (see Section 5.10).

\section{Results: The laws of large numbers.}

3.1. LLN for all semimartingales. We consider here an LLN which holds for all semimartingales, and we start with the version without noise, that is, $Z=X$. For the sake of comparison, we recall the following classical result:

$$
\sum_{i=1}^{\left[t / \Delta_{n}\right]}\left|\Delta_{i}^{n} X\right|^{p} \stackrel{\mathbb{P}}{\longrightarrow} \begin{cases}\sum_{s \leq t}\left|\Delta X_{s}\right|^{p}, & \text { if } p>2, \\ {[X, X]_{t},} & \text { if } p=2 .\end{cases}
$$

Below, and throughout the paper, $g$ always denotes a weight function satisfying (2.7).

Theorem 3.1. For any $t \geq 0$ which is not a fixed time of discontinuity of $X$, we have

$$
\frac{1}{k_{n}} V(X, g, p, 0)_{t}^{n} \stackrel{\mathbb{P}}{\longrightarrow} \begin{cases}\bar{g}(p) \sum_{s \leq t}\left|\Delta X_{s}\right|^{p}, & \text { if } p>2, \\ \bar{g}(2)[X, X]_{t}, & \text { if } p=2,\end{cases}
$$

as soon as $k_{n} \rightarrow \infty$ and $u_{n}=k_{n} \Delta_{n} \rightarrow 0$ [that is, we do not need (2.6) here].

This convergence also holds for any $t$ such that $t / \Delta_{n}$ is an integer for all $n$, if this happens, but it never holds in the Skorokhod sense, except of course when $X$ is continuous. Taking in (2.12) test functions of the form $f(x)=|x|^{p}$ is essential here: the convergence of $\sum_{i=0}^{\left[t / \Delta_{n}\right]-k_{n}} f\left(\bar{X}(g)_{i}^{n}\right)$ for more general $f$ is so far an open question.

Next we have the version with noise, again for an arbitrary semimartingale $X$. In the previous theorem nothing is said about $V(X, g, p, r)_{t}^{n}$ when $r \geq 1$ which are of little interest. However, when noise is present, we need those processes to remove an intrinsic bias, and so we provide their behavior, or at least some (rough) estimates on them. 
TheOREM 3.2. (a) For any $t \geq 0$ which is not a fixed time of discontinuity of $X$ we have

$$
p>2 \text { and }(N-p) \text { holds } \Rightarrow \frac{1}{k_{n}} V(Z, g, p, 0)_{t}^{n} \stackrel{\mathbb{P}}{\longrightarrow} \bar{g}(p) \sum_{s \leq t}\left|\Delta X_{s}\right|^{p} .
$$

Moreover, if $r>0$ and $p+2 r>2$ and if $[N-(p+2 r)]$ holds, then

$$
\text { the sequence }\left(k_{n}^{r-(p+4 r) /(p+2 r)} V(Z, g, p, r)_{t}^{n}\right) \text { is tight. }
$$

(b) Under ( $N$-2) we have for all $t$ as above,

$$
\frac{1}{k_{n}} V(Z, g, 2,0)_{t}^{n}-\frac{1}{2 k_{n}} V(Z, g, 0,1)_{t}^{n} \stackrel{\mathbb{P}}{\longrightarrow} \bar{g}(2)[X, X]_{t}
$$

It is worth emphasizing that the behaviors of $V(Z, g, p, 0)^{n}$ and $V(X, g, p, 0)^{n}$ are basically the same when $p>2$, at least for the convergence in probability because the jumps dominate in these processes both the "continuous martingale part" and the noise, and, in particular, by using the pre-averaging procedure, we wipe out the noise completely in this case. On the opposite, when $p=2$ the two processes $V(Z, g, 2,0)^{n}$ and $V(X, g, 2,0)^{n}$ behave differently, even at the level of convergence in probability.

3.2. LLN for continuous Itô semimartingales - 1 . When $X$ is continuous, Theorem 3.2 gives a vanishing limit when $p>2$, so it is natural in this case to look for a normalization which provides a nontrivial limit. Exactly as when there is no noise (see [13]) this is possible only when $X$ is a continuous Itô semimartingale of the form (2.14).

Theorem 3.3. Assume Hypothesis $(N-q)$ for some $q>2$ and that $X$ is given by (2.14). Assume also that $b$ is locally bounded and that $\sigma$ and $\alpha$ are càdlàg. Then if $0<p \leq q / 2$ we have

$$
\Delta_{n}^{1-p / 4} V(Z, g, p, 0)_{t}^{n} \stackrel{\text { u.c.p. }}{\longrightarrow} m_{p} \int_{0}^{t}\left|\theta \bar{g}(2) \sigma_{s}^{2}+\frac{\bar{g}^{\prime}(2)}{\theta} \alpha_{s}^{2}\right|^{p / 2} d s,
$$

where $m_{p}$ denotes the pth absolute moment of $\mathcal{N}(0,1)$.

This result should be compared to the fact that, under the same assumptions on $X$, the processes $\Delta_{n}^{1-p / 2} \sum_{i=1}^{\left[t / \Delta_{n}\right]}\left|\Delta_{i}^{n} X\right|^{p}$ converge to the limiting process $m_{p} \int_{0}^{t}\left|\sigma_{s}\right|^{p} d s$.

This theorem is not really satisfactory; unlike Theorem 3.2(a), the limit depends on the noise, through $\alpha_{s}$, and further, we do not know how to prove a CLT associated to it because of the intrinsic bias due to the noise (see Remark 2.3). However, at least when $p$ is an even integer (the most 
interesting case in practice), we have a useful substitute. That is, by an application of the binomial formula and the estimation of the terms that involve the process $\alpha_{s}$, we obtain (up to a constant factor) the process $\int_{0}^{t}\left|\sigma_{s}\right|^{p} d s$ in the limit.

For any even integer $p \geq 2$ we introduce the numbers $\rho_{p, l}$ for $l=0, \ldots, p / 2$ which are the solutions of the following triangular system of linear equations $\left(C_{q}^{p}=\frac{q !}{p !(q-p) !}\right.$ denote the binomial coefficients):

$$
\left.\begin{array}{l}
\rho_{p, 0}=1, \\
\sum_{l=0}^{j} 2^{l} m_{2 j-2 l} C_{p-2 l}^{2 j-2 l} \rho_{p, l}=0, \quad j=1,2, \ldots, p / 2 .
\end{array}\right\}
$$

These could, of course, be explicitly computed, and, for example, we have

$$
\rho_{p, 1}=-\frac{1}{2} C_{p}^{2}, \quad \rho_{p, 2}=\frac{3}{4} C_{p}^{4}, \quad \rho_{p, 3}=-\frac{15}{8} C_{p}^{6} .
$$

Then for any process $Y$ and for $p \geq 2$ an even integer we set

$$
\bar{V}(Y, g, p)_{t}^{n}=\sum_{l=0}^{p / 2} \rho_{p, l} V(Y, g, p-2 l, l)_{t}^{n} .
$$

TheOREM 3.4. (a) Let $X$ be an arbitrary semimartingale, and assume $(N-p)$ for some even integer $p \geq 2$. Then for all $t \geq 0$ we have

$$
\frac{1}{k_{n}} \bar{V}(Z, g, p)_{t}^{n} \stackrel{\mathbb{P}}{\longrightarrow} \begin{cases}\bar{g}(p) \sum_{s \leq t}\left|\Delta X_{s}\right|^{p}, & \text { if } p \geq 4, \\ \bar{g}(2)[X, X]_{t}, & \text { if } p=2 .\end{cases}
$$

(b) Let $X$ satisfy (2.14), and assume ( $N-2 p)$ for some even integer $p \geq 2$. Assume also that $b$ is locally bounded and that $\sigma$ and $\alpha$ are càdlàg. Then we have

$$
\Delta_{n}^{1-p / 4} \bar{V}(Z, g, p)_{t}^{n} \stackrel{\text { u.c.p. }}{\longrightarrow} m_{p}(\theta \bar{g}(2))^{p / 2} \int_{0}^{t}\left|\sigma_{s}\right|^{p} d s .
$$

The first part of (3.10) is an obvious consequence of (a) of Theorem 3.2 whereas the second part of (3.10) is nothing other than (3.5) because $\rho_{2,1}=$ $-1 / 2$.

3.3. LLN for continuous Itô semimartingales -2. Statistical applications require "estimators" for the conditional variance which will appear in the CLTs associated with some of the previous LLNs. In other words, we need to provide some other laws of large numbers, which a priori seem artificial but are motivated by potential applications. 
To this end we need auxiliary processes to be used also for the CLTs below. Let $W^{1}$ and $W^{2}$ be two independent Brownian motions on another auxiliary filtered probability space $\left(\Omega^{\prime}, \mathcal{F}^{\prime},\left(\mathcal{F}_{t}^{\prime}\right)_{t \geq 0}, \mathbb{P}^{\prime}\right)$. With any function $g$ satisfying (2.7), and extended as before on $\mathbb{R}$ by setting it to be 0 outside $[0,1]$, we define the following Wiener integral processes:

$$
L(g)_{t}=\int g(s-t) d W_{s}^{1}, \quad L^{\prime}(g)_{t}=\int g^{\prime}(s-t) d W_{s}^{2} .
$$

If $h$ is another function satisfying (2.7), we define $L(h)$ and $L^{\prime}(h)$ likewise, with the same $W^{1}$ and $W^{2}$. The four-dimensional process $U:=\left(L(g), L^{\prime}(g), L(h)\right.$, $\left.L^{\prime}(h)\right)$ is continuous in time, centered, Gaussian and stationary. Clearly $(L(g), L(h))$ is independent of $\left(L^{\prime}(g), L^{\prime}(h)\right)$, and the variables $U_{t}$ and $U_{t+s}$ are independent if $s \geq 1$.

The process $L(g)$ comes in naturally as the limit of $\bar{W}(g)_{i}^{n}$ [that is, $\bar{X}(g)$ when $X=W]$; indeed, we will see that $L(g)_{t}$ is the limit in law of $\frac{1}{\sqrt{u_{n}}} \bar{W}(g)_{\left[\Delta_{n} / t\right]}^{n}$, and we need the whole process $L(g)_{t}$ to account for the dependency of the variables $\bar{W}(g)_{i}^{n}$ when $i$ varies. In the same way, $k_{n} \widehat{\chi}(g)_{\left[\Delta_{n} / t\right]}^{n}$ converges in law to $2\left(\alpha_{t}\right)^{2} L^{\prime}(g)_{t}$ (see the "key Lemma" 5.1 below).

Some further notation is needed. We set

$$
\left.\begin{array}{l}
m_{p}(g ; \eta, \zeta)=\mathbb{E}^{\prime}\left(\left(\eta L(g)_{0}+\zeta L^{\prime}(g)_{0}\right)^{p}\right), \\
m_{p, q}(g, h ; \eta, \zeta) \\
\quad=\int_{0}^{2} \mathbb{E}^{\prime}\left(\left(\eta L(g)_{1}+\zeta L^{\prime}(g)_{1}\right)^{p}\left(\eta L(h)_{t}+\zeta L^{\prime}(h)_{t}\right)^{q}\right) d t .
\end{array}\right\}
$$

These could of course be expressed by the mean of expectations with respect to the joint law of $U$ above and, considered as functions of $(\eta, \zeta)$, they are $C^{\infty}$. In particular, since $L(g)_{0}$ and $L^{\prime}(g)_{0}$ are independent centered Gaussian variables with respective variances $\bar{g}(2)$ and $\bar{g}^{\prime}(2)$, when $p$ in an integer we have

$$
m_{p}(g ; \eta, \zeta)= \begin{cases}\sum_{v=0}^{p / 2} C_{p}^{2 v}\left(\eta^{2} \bar{g}(2)\right)^{v}\left(\zeta^{2} \bar{g}^{\prime}(2)\right)^{p / 2-v} m_{2 v} m_{p-2 v} \\ 0, \quad \text { if } p \text { is even, } \\ 0 \text { is odd. }\end{cases}
$$

Next, recalling (3.7), we set for $p \geq 2$ an even integer:

$$
\left.\begin{array}{c}
\mu_{p}(g ; \eta, \zeta)=\sum_{r=0}^{p / 2} \rho_{p, r}\left(2 \zeta^{2} \bar{g}^{\prime}(2)\right)^{r} m_{p-2 r}(g ; \eta, \zeta), \\
\mu_{2 p}(g, h ; \eta, \zeta)=\sum_{r, r^{\prime}=0}^{p / 2} \rho_{p, r} \rho_{p, r^{\prime}}\left(2 \zeta^{2} \bar{g}^{\prime}(2)\right)^{r}\left(2 \zeta^{2} \bar{h}^{\prime}(2)\right)^{r^{\prime}} \\
\times m_{p-2 r, p-2 r^{\prime}}(g, h ; \eta, \zeta), \\
\bar{\mu}_{2 p}(g, h ; \eta, \zeta)=\mu_{2 p}(g, h ; \eta, \zeta)-2 \mu_{p}(g ; \eta, \zeta) \mu_{p}(h ; \eta, \zeta) .
\end{array}\right\}
$$


The following lemma will be useful in the sequel:

LEMMA 3.5. We have

$$
\mu_{p}(g ; \eta, \zeta)=m_{p} \eta^{p} \bar{g}(2)^{p / 2}
$$

Moreover if $g_{i}$ is a finite family of functions satisfying (2.7), for any $(\eta, \zeta)$ the matrix with entries $\bar{\mu}_{2 p}\left(g_{i}, g_{j} ; \eta, \zeta\right)$ is symmetric nonnegative.

Finally, we associate with any process $Y$ and any even integer $p$ the functionals

$$
\begin{aligned}
& M(Y, g, h ; p)_{t}^{n} \\
& =\sum_{r, r^{\prime}=0}^{p / 2} \rho_{p, r} \rho_{p, r^{\prime}} \sum_{i=0}^{\left[t / \Delta_{n}\right]-3 k_{n}}\left(\widehat{Y}(g)_{i}^{n}\right)^{r}\left(\widehat{Y}(h)_{i}^{n}\right)^{r^{\prime}} \\
& \times\left(\left|\bar{Y}(g)_{i+k_{n}}^{n}\right|^{p-2 r} \frac{1}{k_{n}} \sum_{j=1}^{2 k_{n}}\left|\bar{Y}(h)_{i+j}^{n}\right|^{p-2 r^{\prime}}\right. \\
& \left.-2\left|\bar{Y}(g)_{i}^{n}\right|^{p-2 r}\left|\bar{Y}(h)_{i+k_{n}}^{n}\right|^{p-2 r^{\prime}}\right) .
\end{aligned}
$$

Then our last LLN is as follows:

TheOrem 3.6. Let $X$ satisfy (2.14), and let $p \geq 2$ be an even integer. Assume (N-2p), that $b$ is locally bounded and that $\sigma$ and $\alpha$ are càdlàg. Then if $p \leq q / 2$ and if $g$ and $h$ are two functions satisfying (2.7), we have

$$
\Delta_{n}^{1-p / 2} M(Z, g, h ; p)_{t}^{n} \stackrel{\text { u.c.p. }}{\longrightarrow} \theta^{-p / 2} \int_{0}^{t} \bar{\mu}_{2 p}\left(g, h ; \theta \sigma_{s}, \alpha_{s}\right) d s .
$$

The reader will observe that the limit in (3.18) is symmetrical in $g$ and $h$, although $M(Y, g, h ; p)_{t}^{n}$ is not. The motivation for this result is that it provides consistent estimators for the conditional variance to be encountered in the CLT below (see Remark 4.2). Indeed, as the summands of the $V(Y, g, p, r)_{t}^{n}$ are asymptotically $k_{n}$-dependent in the continuous case (see Remark 2.3), the statistic $\Delta_{n}^{1-p / 2} M(Z, g, h ; p)_{t}^{n}$ is, up to a multiplicative constant, an empirical analogue of the asymptotic conditional covariance between $\bar{V}(Z, g, p)_{t}^{n}$ and $\bar{V}(Z, h, p)_{t}^{n}$. 


\section{Results: The central limit theorems.}

4.1. CLT for continuous Itô semimartingales. As mentioned before, we do not know whether a CLT associated with the convergence (3.6) exists. But there is one associated with (3.11) when $p$ is an even integer. Below we give a joint CLT for several weight functions $g$ at the same time. We use the notation

$$
\widetilde{V}(g, p)_{t}^{n}=\frac{1}{\Delta_{n}^{1 / 4}}\left(\Delta_{n}^{1-p / 4} \bar{V}(Z, g, p)_{t}^{n}-m_{p}(\theta \bar{g}(2))^{p / 2} \int_{0}^{t}\left|\sigma_{s}\right|^{p} d s\right) .
$$

In view of Lemma 3.5, the square-root matrix $\psi$ referred to below exists, and by a standard selection theorem one can find a measurable version for it. For the stable convergence in law used below, we refer, for example, to [16].

TheOrem 4.1. Assume Hypothesis $(K)$ and $(N-4 p)$, where $p$ is an even integer, and also that the processes $\alpha$ and $\beta(3)$ are càdlàg. If $\left(g_{i}\right)_{1 \leq i \leq d}$ is a family of functions satisfying (2.7), for each $t \geq 0$ the variables $\left(\widetilde{V}\left(g_{i}, p\right)_{t}^{n}\right)_{1 \leq i \leq d}$ converge stably in law to the d-dimensional variable,

$$
\left(\theta^{1 / 2-p / 2} \sum_{j=1}^{d} \int_{0}^{t} \psi_{i j}\left(\theta \sigma_{s}, \alpha_{s}\right) d B_{s}^{j}\right)_{1 \leq i \leq d},
$$

where $B$ is a d-dimensional Brownian motion independent of $\mathcal{F}$ (and defined on an extension of the space), and $\psi$ is a measurable $d \times d$ matrix-valued function such that $\left(\psi \psi^{*}\right)(\eta, \zeta)$ is the matrix with entries $\bar{\mu}_{2 p}\left(g_{i}, g_{j} ; \eta, \zeta\right)$, as defined by (3.15).

Up to the multiplicative constant $\theta^{1-p / 2}$, the $\mathcal{F}$-conditional covariance of the $j$ th and $k$ th components of (4.2) is exactly the right-hand side of (3.18) for $g=g_{j}$ and $h=g_{k}$.

REMARK 4.2. An application of Theorem 3.6 and the properties of stable convergence give now a a feasible version of Theorem 4.1. We obtain, for example, that the quantity

$$
\frac{\tilde{V}(g, p)_{t}^{n}}{\sqrt{\theta^{1-p / 2} \Delta_{n}^{1-p / 2} M(Z, g, g ; p)_{t}^{n}}}
$$

converges stably in law (for any fixed $t$ ) to a variable $U \sim \mathcal{N}(0,1)$ independent of $\mathcal{F}$. The latter can be used to construct confidence regions for the quantity $\int_{0}^{t}\left|\sigma_{s}\right|^{p} d s$ for even $p$ 's. 
REMARK 4.3. Theorem 4.1 can be extended to the convergence along finite families of times, but we do not know whether a functional convergence holds, although it is quite likely.

4.2. CLT for discontinuous Itô semimartingales. Now we turn to the case when $X$ jumps. There is a CLT for Theorem 3.2, at least when $p=2$ and $p>3$, exactly as in [13] for the processes of type (3.1). The CLT for Theorem 3.4 , when $p$ is an even integer, takes the same form. In this subsection we are interested in the case $p>3$, whereas the case $p=2$ is dealt with in the next subsection.

In view of statistical applications (see Remark 4.5 below), and as in the previous subsection, we need to consider a family $\left(g_{i}\right)_{1 \leq i \leq d}$ of weight functions. We use the notation

$$
\widetilde{V}^{\star}(g, p)_{t}^{n}=\frac{1}{\Delta_{n}^{1 / 4}}\left(\frac{1}{k_{n}} V(Z, g, p, 0)_{t}^{n}-\bar{g}(p) \sum_{s \leq t}\left|\Delta X_{s}\right|^{p}\right)
$$

and, further, when $p \geq 4$ is an even integer,

$$
\bar{V}^{\star}(g, p)_{t}^{n}=\frac{1}{\Delta_{n}^{1 / 4}}\left(\frac{1}{k_{n}} \bar{V}(Z, g, p)_{t}^{n}-\bar{g}(p) \sum_{s \leq t}\left|\Delta X_{s}\right|^{p}\right) .
$$

These are the processes whose asymptotic behavior is studied, but to describe the limit we need some rather cumbersome notation which involves the $d$ weight functions, $g_{j}$ satisfying (2.7). For any real $x$ and any $p>0$ we write $\{x\}^{p}=|x|^{p} \operatorname{sign}(x)$, and we introduce four $d \times d$ symmetric matrices $\Psi_{p-}, \Psi_{p+}, \bar{\Psi}_{p-}$ and $\bar{\Psi}_{p+}$ with entries:

$$
\begin{aligned}
\Psi_{p-}^{i j}=\int_{0}^{1} & \left(\int_{t}^{1}\left\{g_{i}(s)\right\}^{p-1} g_{i}(s-t) d s\right) \\
& \times\left(\int_{t}^{1}\left\{g_{j}(s)\right\}^{p-1} g_{j}(s-t) d s\right) d t, \\
\Psi_{p+}^{i j}=\int_{0}^{1} & \left(\int_{0}^{1-t}\left\{g_{i}(s)\right\}^{p-1} g_{i}(s+t) d s\right) \\
& \times\left(\int_{0}^{1-t}\left\{g_{j}(s)\right\}^{p-1} g_{j}(s+t) d s\right) d t, \\
\Psi_{p-}^{i j}=\int_{0}^{1} & \left(\int_{t}^{1}\left\{g_{i}(s)\right\}^{p-1} g_{i}^{\prime}(s-t) d s\right) \\
& \times\left(\int_{t}^{1}\left\{g_{j}(s)\right\}^{p-1} g_{j}^{\prime}(s-t) d s\right) d t, \\
\bar{\Psi}_{p+}^{i j}=\int_{0}^{1} & \left(\int_{0}^{1-t}\left\{g_{i}(s)\right\}^{p-1} g_{i}^{\prime}(s+t) d s\right) \\
\times & \left(\int_{0}^{1-t}\left\{g_{j}(s)\right\}^{p-1} g_{j}^{\prime}(s+t) d s\right) d t .
\end{aligned}
$$


These matrices are semi-definite positive, and we can thus consider four independent sequences of i.i.d. $d$-dimensional variables $\left(U_{m-}\right)_{m \geq 1},\left(U_{m+}\right)_{m \geq 1}$, $\left(\bar{U}_{m-}\right)_{m \geq 1}$ and $\left(\bar{U}_{m+}\right)_{m \geq 1}$, defined on an extension of the space, independent of $\mathcal{F}$, and such that for each $m$ the $d$-dimensional variables $U_{m-}, U_{m+}$, $\bar{U}_{m-}$ and $\bar{U}_{m+}$ are centered Gaussian vectors with respective covariances $\Psi_{p-}, \Psi_{p+}, \bar{\Psi}_{p-}$ and $\bar{\Psi}_{p+}$. Note that these variables also depend on $p$ and on the family $\left(g_{j}\right)$, although it does not show in the notation.

Now let $\left(T_{m}\right)_{m \geq 1}$ be a sequence of stopping times with pairwise disjoint graphs, such that $\Delta X_{t} \neq 0$ implies that $t=T_{m}$ for some $m$. As is well known (see [13]), the following $d$-dimensional processes are well defined when $p>3$ and $\alpha$ is càdlàg, and are $\mathcal{F}$-conditional martingales:

$$
\begin{aligned}
U(p)_{t}=p \sum_{m \geq 1}\left\{\Delta X_{T_{m}}\right\}^{p-1} & \left(\sqrt{\theta} \sigma_{T_{m}-} U_{m-}\right. \\
& +\frac{\alpha_{T_{m}-}}{\sqrt{\theta}} \bar{U}_{m-} \\
& \left.+\sqrt{\theta} \sigma_{T_{m}} U_{m+}+\frac{\alpha_{T_{m}}}{\sqrt{\theta}} \bar{U}_{m+}\right) 1_{\left\{T_{m} \leq t\right\}} .
\end{aligned}
$$

Moreover, although these processes obviously depend on the choice of the times $T_{m}$, their $\mathcal{F}$-conditional laws do not; so if the stable convergence in law below holds for a particular "version" of $U(p)_{t}$, it also holds for all other versions.

Theorem 4.4. Assume Hypothesis (H) and let $p>3$. Assume also ( $N$ $2 p$ ) and that the process $\alpha$ is càdlàg. If $\left(g_{i}\right)_{1 \leq i \leq d}$ is a family of functions satisfying (2.7), for each $t \geq 0$ the variables $\left(\widetilde{V}^{*}\left(g_{i}, p\right)_{t}^{n}\right)_{1 \leq i \leq d}$ converge stably in law to the $d$-dimensional variable $U(p)_{t}$.

The same holds for the sequence $\left(\bar{V}^{*}\left(g_{i}, p\right)_{t}^{n}\right)_{1 \leq i \leq d}$ if further $p$ is an even integer.

REMARK 4.5. In the spirit of [4], we can use this result to test for the presence of jumps in the presence of noise. We choose two distinct onedimensional weight functions $g$ and $h$. It follows from Theorem 3.4 that, taking, for example, $p=4$,

$\frac{\bar{V}(Z, g, 4)_{t}^{n}}{\bar{V}(Z, h, 4)_{t}^{n}} \stackrel{\mathbb{P}}{\longrightarrow} \begin{cases}\bar{g}(2)^{2} / \bar{h}(2)^{2}, & \text { on the set where } X \text { is continuous on }[0, t], \\ \bar{g}(4) / \bar{h}(4), & \text { on the set where } X \text { has jumps on }[0, t] .\end{cases}$

We can choose $g$ and $h$ such that the two limits above are different. Then Theorems 4.1 and 4.4 provide central limit theorems for the statistics $\bar{V}(Z, g, 4)_{t}^{n} / \bar{V}(Z, h, 4)_{t}^{n}$ in both occurrences, allowing for feasible testing of the two hypotheses. For instance, when $X$ is continuous, we deduce that the sequence

$$
\Delta_{n}^{-1 / 4}\left(\frac{\bar{V}(Z, g, 4)_{t}^{n}}{\bar{V}(Z, h, 4)_{t}^{n}}-\frac{\bar{g}(2)^{2}}{\bar{h}(2)^{2}}\right)
$$


converges stably in law toward a mixed normal random variable with $\mathcal{F}^{(0)}$ _ conditional variance,

$$
\begin{aligned}
& \theta^{-3}\left(3(\theta \bar{h}(2))^{2} \int_{0}^{t} \sigma_{s}^{4} d s\right)^{-2}\left(1,-\frac{\bar{g}(2)^{2}}{\bar{h}(2)^{2}}\right) \\
& \quad \times\left(\bar{\mu}_{8}\left(g_{i}, g_{j} ; \eta, \zeta\right)\right)_{1 \leq i \leq 2,1 \leq j \leq 2}\left(1,-\frac{\bar{g}(2)^{2}}{\bar{h}(2)^{2}}\right)^{\star},
\end{aligned}
$$

where the $(2 \times 2)$-matrix $\left(\bar{\mu}_{8}\left(g_{i}, g_{j} ; \eta, \zeta\right)\right)_{1 \leq i \leq 2,1 \leq j \leq 2}$ is defined by $(3.15)$ and $g_{1}=g, g_{2}=h$. Since we are able to consistently estimate the above quantity by virtue of Theorems 3.4 and 3.6, we can immediately obtain a feasible test for the null hypothesis of no jumps.

4.3. CLT for the quadratic variation. Finally we give a CLT for the quadratic variation associated with (3.5) when $p=2$ or, equivalently, with (3.10) which is exactly the same in this case. In contrast to the preceding results the function $g$ is kept fixed; thus we will only show a one-dimensional result. So the processes of interest are simply

$$
\bar{V}_{t}^{n}=\frac{1}{\Delta_{n}^{1 / 4}}\left(\frac{1}{k_{n}} \bar{V}(Z, g, 2)_{t}^{n}-\bar{g}(2)[X, X]_{t}\right) .
$$

In order to describe the limit, we introduce an extension of the space on which are defined a Brownian motion $B$ and variables $U_{m-}, \bar{U}_{m-}, U_{m+}, \bar{U}_{m+}$ indexed by $m \geq 1$; each of these being independent from the others and independent of $\mathcal{F}$, and such that the variables $U_{m-}, U_{m+}, \bar{U}_{m-}, \bar{U}_{m+}$ are centered Gaussian variables with respective variances $\Psi_{2-}^{11}, \Psi_{2+}^{11}, \bar{\Psi}_{2-}^{11}$ and $\bar{\Psi}_{2+}^{11}$, as defined in (4.5).

As in the previous section, $\left(T_{m}\right)_{m \geq 1}$ is a sequence of stopping times with pairwise disjoint graphs, such that $\Delta X_{t} \neq 0$ implies that $t=T_{m}$ for some $m$. Then we associate with these data the process $U(2)$ as defined by (4.6). The result goes as follows:

Theorem 4.6. Assume Hypothesis (H). Assume also (N-4) and that the process $\alpha$ is càdlàg. Then for each t the variables $\bar{V}_{t}^{n}$ converge stably in law to the variable

$$
\bar{U}_{t}=\theta^{-1 / 2} \int_{0}^{t} \sqrt{\bar{\mu}_{4}\left(g, g ; \theta \sigma_{s}, \alpha_{s}\right)} d B_{s}+U(2)_{t},
$$

where $\bar{\mu}_{4}(g, g ; \eta, \zeta)$ is defined by (3.15) which here takes the form

$$
\begin{aligned}
\bar{\mu}_{4}(g, g ; \eta, \zeta)=4 \int_{0}^{1} & \left(\eta^{2} \int_{s}^{1} g(u) g(u-s) d u\right. \\
& \left.+\zeta^{2} \int_{s}^{1} g^{\prime}(u) g^{\prime}(u-s) d u\right)^{2} d s .
\end{aligned}
$$


When further $X$ is continuous, the processes $\bar{V}^{n}$ converge stably (in the functional sense) to the process (4.8) with $U(2)=0$ in this case.

When $X$ is continuous, we exactly recover Theorem 4.1 when $d=1$ and $g_{1}=g$, for $p=2$. Note that we do not need Hypothesis $(\mathrm{K})$ here because of the special feature of the case $p=2$. When $X$ has jumps, however, the functional convergence does not hold.

EXAMPLE 4.7. Notice that the limiting variable $\bar{U}_{t}$ is mixed normal with $\mathcal{F}^{(0)}$-conditional variance,

$$
\begin{aligned}
& \theta^{-1} \int_{0}^{t} \bar{\mu}_{4}\left(g, g ; \theta \sigma_{s}, \alpha_{s}\right) d s \\
& +4 \sum_{m \geq 1}\left|\Delta X_{T_{m}}\right|^{2}\left(\theta \sigma_{T_{m}-}^{2} \Psi_{2-}+\frac{\alpha_{T_{m}-}^{2}}{\theta} \bar{\Psi}_{2-}\right. \\
& \left.+\theta \sigma_{T_{m}}^{2} \Psi_{2+}+\frac{\alpha_{T_{m}}^{2}}{\theta} \bar{\Psi}_{2+}\right) 1_{\left\{T_{m} \leq t\right\}} .
\end{aligned}
$$

For the sake of demonstration let us consider the weight function $g(x)=$ $\min (x, 1-x) 1_{\{0 \leq x \leq 1\}}$. In this case we obtain

$$
\Psi_{2+}=\Psi_{2-}=\frac{151}{80640}, \quad \bar{\Psi}_{2+}=\bar{\Psi}_{2-}=\frac{1}{96}
$$

and

$$
\bar{\mu}_{4}(g, g ; \eta, \zeta)=4\left(\frac{151}{80640} \eta^{4}+\frac{1}{48} \eta^{2} \zeta^{2}+\frac{1}{6} \zeta^{4}\right) .
$$

5. The proofs. It is difficult to describe the scheme of the proofs in a few words, since they are quite technical. However, we can state the basic ideas:

- For the case $p>2$ of Theorem 3.1 and Theorems 3.2 and 4.4, the "big" jumps play the leading role, and so the results are proved first when all jumps are bigger than some $\varepsilon>0$ (hence there are finitely many of them); we thus examine what happens around each jump, and show that the rest is negligible.

- For the continuous case, we use the approximations

$$
\bar{Z}(g)_{i}^{n} \approx \sigma_{i \Delta_{n}} \bar{W}(g)_{i}^{n}+\bar{\chi}(g)_{i}^{n}, \quad \widehat{Z}(g)_{i}^{n} \approx 2 \bar{g}^{\prime}(2) \alpha_{i \Delta_{n}}^{2} / k_{n} .
$$

Since the approximating quantities in (5.1) are asymptotically $k_{n}$-dependent we apply the block splitting technique to prove Theorem 4.1. Precisely, we split the sum over $i$ in the definition of $\bar{V}(Z, g, p)_{t}^{n}$ into big blocks of size $m k_{n}$ which are separated by small blocks of size $k_{n}$. The big blocks become asymptotically conditionally independent, and the small blocks become negligible as $m \rightarrow \infty$. In a second step we prove a CLT for big blocks, for any fixed $m$. 
- For the quadratic variation (case $p=2$ of Theorem 3.1 and Theorem 4.6) the proof is a sort of mixture of the two approaches.

In the whole proof $K$ denotes a constant which may change from line to line. It may depend on the characteristics of the process $X$ and the law of the noise $\chi$ on $\theta$ and the two sequences, $\left(k_{n}\right)_{n \geq 1}$ and $\left(\Delta_{n}\right)_{n \geq 1}$, but neither on $n$ itself, nor on the index $i$ of the increments $\Delta_{i}^{n} X$ or $\Delta_{i}^{n} Z$. If it depends on an additional parameter $q$; we write it $K_{q}$.

For the proof of all the results we can use a localization procedure, described in detail in [13], for instance, and which allows us to systematically replace the Hypotheses $(N-q),(\mathrm{H})$ or $(\mathrm{K})$, according to the case, by the following strengthened versions:

Hypothesis $(\mathrm{SN}-q)$. We have Hypothesis $(N-q)$, and further $\int Q_{t}\left(\omega^{(0)}\right.$, $d z)|z|^{q} \leq K$.

Hypothesis $(\mathrm{SH})$. We have Hypothesis $(H)$, and the processes $b_{t}, \sigma_{t}$, $\sup _{z \in E}|\delta(t, z)| / \gamma(z)$ and $X$ are bounded.

Hypothesis (SK). We have Hypothesis $(K)$, and the processes $b_{t}, \sigma_{t}$, $\widetilde{b}_{t}, a_{t}, a_{t}^{\prime}, \widetilde{\sigma}_{t}$ and $X$ are bounded.

Observe that under Hypothesis (SK), and upon taking $v$ large enough in (2.15) (changing $v$ changes the coefficients $\widetilde{b}_{t}$ and $a_{t}$ without altering their boundedness), we can also suppose that the last term in (2.15) vanishes identically; that is,

$$
\sigma_{t}=\sigma_{0}+\int_{0}^{t} \widetilde{b}_{s} d s+\int_{0}^{t} \widetilde{\sigma}_{s} d W_{s}+M_{t} .
$$

Recall that $\left|g_{j}^{\prime n}\right| \leq K / k_{n}$. Then the fact that conditionally on $\mathcal{F}^{(0)}$ the $\chi_{t}$ 's are independent and centered, plus Hölder's inequality, gives us that under Hypothesis $(\mathrm{SN}-q)$ we have [the $\sigma$-fields $\mathcal{F}_{i}^{n}$ and $\mathcal{G}_{i}^{n}$ have been defined after (2.11)]

$$
\left.\begin{array}{l}
p \leq q \quad \Rightarrow \quad \mathbb{E}\left(\left|\bar{\chi}(g)_{i}^{n}\right|^{p} \mid \mathcal{G}_{i}^{n}\right) \leq K_{p} k_{n}^{-p / 2}, \\
2 r \leq q \quad \Rightarrow \quad \mathbb{E}\left(\left|\widehat{\chi}(g)_{i}^{n}\right|^{r} \mid \mathcal{G}_{i}^{n}\right) \leq K_{r} k_{n}^{-r} .
\end{array}\right\}
$$

We will also often use the following property, valid for all semimartingales $Y$ :

$$
\begin{aligned}
\bar{Y}(g)_{i}^{n}=\int_{i \Delta_{n}}^{i \Delta_{n}+u_{n}} g_{n}\left(s-i \Delta_{n}\right) d Y_{s} \\
\text { where } g_{n}(s)=\sum_{j=1}^{k_{n}-1} g_{j}^{n} 1_{\left((j-1) \Delta_{n}, j \Delta_{n}\right]}(s) .
\end{aligned}
$$


5.1. Proof of Theorem 3.1. We start with an arbitrary semimartingale $X$, written as (2.1). We more or less follow the scheme of the proof of Theorem 2.2 of [13], and we use the simplifying notation $V(Y, p)^{n}=V(Y, g, p, 0)^{n}$ and $\bar{Y}_{i}^{n}=\bar{Y}(g)_{i}^{n}$. The basic idea follows: for $\varepsilon \in(0,1]$, we set

$$
\left.\begin{array}{lc}
X(\varepsilon)=\left(x 1_{\{|x|>\varepsilon\}}\right) \star \mu, & M(\varepsilon)=\left(x 1_{\{|x| \leq \varepsilon\}}\right) \star(\mu-\nu), \\
A(\varepsilon)=\langle M(\varepsilon), M(\varepsilon)\rangle, & B(\varepsilon)=B-\left(x 1_{\{\varepsilon<|x| \leq 1\}}\right) \star \nu, \\
A^{\prime}(\varepsilon)=\left(x^{2} 1_{\{|x| \leq \varepsilon\}}\right) \star \nu, & B^{\prime}(\varepsilon)=\text { variation process of } B(\varepsilon),
\end{array}\right\}
$$

so that we have

$$
X=X_{0}+B(\varepsilon)+X^{c}+M(\varepsilon)+X(\varepsilon) .
$$

Then we basically show that $\frac{1}{k_{n}} V(B(\varepsilon), p)^{n}$ and $\frac{1}{k_{n}} V(M(\varepsilon), p)^{n}$ are "negligible" when $n \rightarrow \infty$ and $\varepsilon \rightarrow 0$, as well as $\frac{1}{k_{n}} V\left(X^{c}, p\right)^{n}$ when $p>2$ whereas $\frac{1}{k_{n}} V(X(\varepsilon), p)^{n}$ converges to $\bar{g}(p) \sum_{s \leq t}\left|\Delta X_{s}\right|^{p} 1_{\left\{\left|\Delta X_{s}\right|>\varepsilon\right\}}$ and $\frac{1}{k_{n}} V\left(X^{c}, 2\right)^{n}$ converges to $\bar{g}(2) C$ where $C=\left\langle X^{c}, X^{c}\right\rangle$.

Step 1. Let $B^{\prime}$ be the variation process of $B$. The process $B^{\prime}+C+\left(x^{2} \wedge\right.$ $1) \star \nu$ is predictable, increasing finite-valued and hence locally bounded. By an obvious localization procedure it is enough to prove the result under the assumption that, for some constant $K$,

$$
B_{\infty}^{\prime}+C_{\infty}+\left(x^{2} \wedge 1\right) \star \nu_{\infty} \leq K .
$$

We also denote by $T_{n}(\varepsilon)$ the successive jump times of $X(\varepsilon)$ with the convention $T_{0}(\varepsilon)=0$ (which of course is not a jump time). If $0<\varepsilon<\eta \leq 1$, we have

$$
\left.\begin{array}{l}
A(\varepsilon) \leq A^{\prime}(\varepsilon), \quad \Delta B^{\prime}(\varepsilon) \leq \varepsilon, \quad|\Delta M(\varepsilon)| \leq 2 \varepsilon, \\
B^{\prime}(\varepsilon) \leq B^{\prime}+\frac{1}{\varepsilon} A^{\prime}(\eta)+\frac{1}{\eta}\left(x^{2} \wedge 1\right) \star \nu .
\end{array}\right\}
$$

We set $\theta(Y, u, t)=\sup _{s \leq r \leq s+u, r \leq t}\left|Y_{r}-Y_{s}\right|$. Observe that $\bar{Y}_{i}^{n}=-\sum_{j=1}^{k_{n}}(g((j+$ $\left.\left.1) / k_{n}\right)-g\left(j / k_{n}\right)\right)\left(Y_{(i+j) \Delta_{n}}-Y_{i \Delta_{n}}\right)$. Hence, since the derivative $g^{\prime}$ is bounded, we obtain

$$
i \leq\left[t / \Delta_{n}\right]-k_{n}+1 \quad \Rightarrow \quad\left|\bar{Y}_{i}^{n}\right| \leq K \theta\left(Y, u_{n}, t\right) .
$$

Step 2. Here we study $B(\varepsilon)$. (5.9) and $\theta(B(\varepsilon), u, t) \leq \theta\left(B^{\prime}(\varepsilon), u, t\right)$ yield for $p>1$

$$
V(B(\varepsilon), p)_{t}^{n} \leq K k_{n} B^{\prime}(\varepsilon)_{t} \theta\left(B^{\prime}(\varepsilon), u_{n}, t\right)^{p-1} .
$$

Since $\Delta B^{\prime}(\varepsilon) \leq \varepsilon$ we have $\limsup _{n \rightarrow \infty} \theta\left(B^{\prime}(\varepsilon), u_{n}, t\right) \leq \varepsilon$, so by $(5.7)$ and (5.8) we have $\limsup _{n} \frac{1}{k_{n}} V\left(B^{\prime}(\varepsilon), p\right)_{t}^{n} \leq K \varepsilon^{p-1}\left(\frac{1}{\eta}+\frac{1}{\varepsilon} A^{\prime}(\eta)_{t}\right)$ for all $0<\varepsilon<$ $\eta \leq 1$. Since $A^{\prime}(\eta)_{t} \rightarrow 0$ as $\eta \rightarrow 0$, we deduce (choose first $\eta$ small, then $\varepsilon$ smaller) that for $p \geq 2$,

$$
\lim _{\varepsilon \rightarrow 0} \limsup _{n} \frac{1}{k_{n}} V(B(\varepsilon), p)_{t}^{n}=0 .
$$


Step 3. In this step, we consider a square-integrable martingale $Y$ such that $D=\langle Y, Y\rangle$ is bounded. By (5.4),

$$
\mathbb{E}\left(\left(\bar{Y}_{i}^{n}\right)^{2}\right)=\mathbb{E}\left(\int_{i \Delta_{n}}^{i \Delta_{n}+u_{n}} g_{n}\left(s-i \Delta_{n}\right)^{2} d D_{s}\right) \leq K \mathbb{E}\left(D_{i \Delta_{n}+u_{n}}-D_{i \Delta_{n}}\right) .
$$

On the other hand, $\mathbb{E}\left(\bar{Y}_{i}^{n} \bar{Y}_{i+j}^{n}\right)=0$ whenever $j \geq k_{n}$. Therefore,

$$
\mathbb{E}\left(\left(V(Y, 2)_{t}^{n}\right)^{2}\right) \leq k_{n} \sum_{i=0}^{\left[t / \Delta_{n}\right]-k_{n}} \mathbb{E}\left(\left(\bar{Y}_{i}^{n}\right)^{2}\right) \leq K k_{n}^{2} \mathbb{E}\left(D_{t}\right) .
$$

We first apply this with $Y=M(\varepsilon)$, hence $D=A(\varepsilon)$. In view of (5.11) and since $A^{\prime}(\varepsilon)_{t} \rightarrow 0$ as $\varepsilon \rightarrow 0$ and $A^{\prime}(\varepsilon)_{t} \leq K$, we deduce

$$
\lim _{\varepsilon \rightarrow 0} \sup _{n} \mathbb{E}\left(\left(\frac{1}{k_{n}} V(M(\varepsilon), 2)_{t}^{n}\right)^{2}\right)=0 .
$$

Since by (5.9) we have $V(M(\varepsilon), p)_{t}^{n} \leq K V(M(\varepsilon), 2)_{t}^{n} \theta\left(M(\varepsilon), u_{n}, t\right)^{p-2}$ when $p>2$, and since $\limsup \theta\left(M(\varepsilon), u_{n}, t\right) \leq 2 \varepsilon$, we get for $p \geq 2$,

$$
p \geq 2, \eta>0 \Rightarrow \lim _{\varepsilon \rightarrow 0} \limsup _{n \rightarrow \infty} \mathbb{P}\left(\frac{1}{k_{n}} V(M(\varepsilon), p)_{t}^{n}>\eta\right)=0 .
$$

Next, (5.11) with $Y=X^{c}$ yields that the sequence $\frac{1}{k_{n}} V\left(X^{c}, 2\right)_{t}^{n}$ is bounded in $\mathbb{L}^{2}$. Using exactly the same argument as above, where now $\theta\left(X^{c}, u_{n}, t\right) \rightarrow$ 0 , yields

$$
p>2, \eta>0 \Rightarrow \lim _{\varepsilon \rightarrow 0} \limsup _{n \rightarrow \infty} \mathbb{P}\left(\frac{1}{k_{n}} V\left(X^{c}, p\right)_{t}^{n}>\eta\right)=0 .
$$

Step 4 . In this step we study $V(X(\varepsilon), p)_{t}^{n}$. We fix $t>0$ such that $\mathbb{P}\left(\Delta X_{t} \neq\right.$ $0)=0$. For any $m \geq 1$ we set

$$
I(m, n, \varepsilon)=\inf \left(i: i \Delta_{n} \geq T_{m}(\varepsilon)\right) .
$$

Let $\Omega_{n}(t, \varepsilon)$ be the set on which two successive jumps of $X(\varepsilon)$ in $[0, t]$ are more than $u_{n}$ apart, and also $\left[0, u_{n}\right)$ and $\left[t-u_{n}, t\right]$ contain no jump. Then $u_{n} \rightarrow 0$ and $\mathbb{P}\left(\Delta X_{t} \neq 0\right)=0$ yield $\Omega_{n}(t, \varepsilon) \rightarrow \Omega$ a.s. as $n \rightarrow \infty$. On the set $\Omega_{n}(t, \varepsilon)$ we have for $i \leq\left[t / \Delta_{n}\right]-k_{n}+1$,

$$
\overline{X(\varepsilon)}_{i}^{n}= \begin{cases}g_{I(m, n, \varepsilon)-i}^{n} \Delta X_{T_{m}(\varepsilon)}, & \text { if } I(m, n, \varepsilon)-k_{n}+1 \leq i \leq I(m, n, \varepsilon)-1 \\ 0, & \text { for some } m, \\ & \text { otherwise. }\end{cases}
$$

Hence

$$
V(X(\varepsilon), p)_{t}^{n}=\bar{g}(p)_{n} \sum_{s \leq t}\left|\Delta X_{s}\right|^{p} 1_{\left\{\left|\Delta X_{s}\right|>\varepsilon\right\}} \quad \text { on } \Omega_{n}(t, \varepsilon)
$$


and (2.10) yields

$$
\frac{1}{k_{n}} V(X(\varepsilon), p)_{t}^{n} \rightarrow \bar{g}(p) \sum_{s \leq t}\left|\Delta X_{s}\right|^{p} 1_{\left\{\left|\Delta X_{s}\right|>\varepsilon\right\}} .
$$

Step 5. In this step we study $V\left(X^{c}, 2\right)_{t}^{n}$. Set $X^{c}(n, i)_{s}=\int_{i \Delta_{n}}^{s} g_{n}\left(r-i \Delta_{n}\right) d X_{r}^{c}$ when $s>i \Delta_{n}$. Using (5.4) and Itô's formula, we get $\left(\bar{X}_{i}^{c, n}\right)^{2}=\zeta_{i}^{n}+\zeta_{i}^{\prime n}$ where

$$
\zeta_{i}^{n}=\int_{i \Delta_{n}}^{i \Delta_{n}+u_{n}} g_{n}\left(s-i \Delta_{n}\right)^{2} d C_{s}, \quad \zeta_{i}^{\prime n}=2 \int_{i \Delta_{n}}^{i \Delta_{n}+u_{n}} X^{c}(n, i)_{s} d X_{s}^{c} .
$$

On one hand, $\sum_{i=0}^{\left[t / \Delta_{n}\right]-k_{n}} \zeta_{i}^{n}$ is equal to $\bar{g}(2)_{n} C_{t}$ plus a term smaller in absolute value than $K C_{u_{n}}$ and another term smaller than $K\left(C_{t}-C_{t-u_{n}}\right)$. Then, obviously,

$$
\frac{1}{k_{n}} \sum_{i=0}^{\left[t / \Delta_{n}\right]-k_{n}} \zeta_{i}^{n} \rightarrow \bar{g}(2) C_{t}
$$

On the other hand, we have $\mathbb{E}\left(\zeta_{i}^{\prime n} \zeta_{i+j}^{\prime n}\right)=0$ when $j \geq k_{n}$, and

$$
\mathbb{E}\left(\left(\zeta_{i}^{\prime n}\right)^{2}\right) \leq 4 \mathbb{E}\left(\left(C_{i \Delta_{n}+u_{n}}-C_{i \Delta_{n}}\right) \sup _{s \in\left[i \Delta_{n}, i \Delta_{n}+u_{n}\right]} X^{c}(n, i)_{s}^{2}\right) .
$$

By Doob's inequality, $\mathbb{E}\left(\sup _{s \in\left[i \Delta_{n}, i \Delta_{n}+u_{n}\right]} X^{c}(n, i)_{s}^{4}\right) \leq K \mathbb{E}\left(\left(C_{i \Delta_{n}+u_{n}}-C_{i \Delta_{n}}\right)^{2}\right)$, hence the Cauchy-Schwarz inequality yields

$$
\mathbb{E}\left(\left(\zeta_{i}^{\prime n}\right)^{2}\right) \leq K \mathbb{E}\left(\left(C_{i \Delta_{n}+u_{n}}-C_{i \Delta_{n}}\right)^{2}\right) \leq K \mathbb{E}\left(\left(C_{i \Delta_{n}+u_{n}}-C_{i \Delta_{n}}\right) \theta\left(C, u_{n}, t\right)\right),
$$

whenever $i \leq\left[t / \Delta_{n}\right]-k_{n}+1$. At this point, the same argument used in (5.11) gives

$$
\mathbb{E}\left(\left(\sum_{i=0}^{\left[t / \Delta_{n}\right]-k_{n}} \zeta_{i}^{\prime n}\right)^{2}\right) \leq K k_{n}^{2} \mathbb{E}\left(C_{t} \theta\left(C, u_{n}, t\right)\right) \leq K k_{n}^{2} \mathbb{E}\left(\theta\left(C, u_{n}, t\right)\right) .
$$

But $\theta\left(C, u_{n}, t\right)$ tends to 0 and is smaller uniformly in $n$ than a squareintegrable variable. We then deduce that $\frac{1}{k_{n}} \sum_{i=0}^{\left[t / \Delta_{n}\right]-k_{n}} \zeta_{i}^{\prime n} \stackrel{\mathbb{P}}{\longrightarrow} 0$ which, combined with (5.15), yields

$$
\frac{1}{k_{n}} V\left(X^{c}, 2\right)_{t}^{n} \stackrel{\mathbb{P}}{\longrightarrow} \bar{g}(2) C_{t} .
$$

Step 6. It remains to put all the previous partial results together. For this we use the following obvious property: for any $p \geq 2$ and $\eta>0$ there is a constant $K_{p, \eta}$ such that

$$
x, y \in \mathbb{R} \quad \Rightarrow \quad|| x+\left.y\right|^{p}-\left.|x|^{p}\left|\leq K_{p, \eta}\right| y\right|^{p}+\eta|x|^{p} .
$$


Suppose first that $p>2$. Applying (5.17) and (5.6), we get

$$
\begin{aligned}
& \left|V(X, p)_{t}^{n}-V(X(\varepsilon), p)_{t}^{n}\right| \\
& \quad \leq \eta V(X(\varepsilon), p)_{t}^{n}+K_{p, \eta}\left(V(B(\varepsilon), p)_{t}^{n}+V\left(X^{c}, p\right)_{t}^{n}+V(M(\varepsilon), p)_{t}^{n}\right) .
\end{aligned}
$$

Then by (5.10), (5.12), (5.13) and (5.14), plus $\sum_{s \leq t}\left|\Delta X_{s}\right|^{p} 1_{\left\{\left|\Delta X_{s}\right|>\varepsilon\right\}} \rightarrow$ $\sum_{s \leq t}\left|\Delta X_{s}\right|^{p}$ as $\varepsilon \rightarrow 0$, and by taking $\eta$ arbitrarily small in the above, we obtain the first part of (3.2).

Next suppose that $p=2$. The same argument shows that it is enough to prove that

$$
\frac{1}{k_{n}} V\left(X^{c}+X(\varepsilon), 2\right)_{t}^{n} \stackrel{\mathbb{P}}{\longrightarrow} \bar{g}(2)\left(C_{t}+\sum_{s \leq t}\left|\Delta X_{s}\right|^{2} 1_{\left\{\left|\Delta X_{s}\right|>\varepsilon\right\}}\right) .
$$

On the set $\Omega_{n}(t, \varepsilon)$, one easily sees that

$$
V\left(X^{c}+X(\varepsilon), 2\right)_{t}^{n}=V\left(X^{c}, 2\right)_{t}^{n}+V(X(\varepsilon), 2)_{t}^{n}+\sum_{m \geq 1: T_{m}(\varepsilon) \leq t} \zeta_{m}^{n},
$$

where

$$
\begin{aligned}
\zeta_{m}^{n} & =\sum_{i=I(m, n, \varepsilon)-k_{n}+1}^{I(m, n, \varepsilon)-1} \zeta(m, n, i), \\
\zeta(m, n, i) & =\left|g_{I(m, n, \varepsilon)-i}^{n} \Delta X_{T_{m}(\varepsilon)}+\bar{X}_{i}^{c, n}\right|^{2}-\left|g_{I(m, n, \varepsilon)-i}^{n} \Delta X_{T_{m}(\varepsilon)}\right|^{2}-\left|\bar{X}_{i}^{c, n}\right|^{2} .
\end{aligned}
$$

In view of (5.9), we deduce from (5.17) that for all $\eta>0$,

$$
|\zeta(m, n, i)| \leq K_{\eta} \theta\left(X^{c}, u_{n}, t\right)^{2}+K \eta\left|\Delta X_{T_{m}(\varepsilon)}\right|^{2},
$$

if $I(m, n, \varepsilon)-k_{n}<i<I(m, n, \varepsilon)$ and $T_{m}(\varepsilon) \leq t$. Since $\eta$ is arbitrarily small, $\zeta_{m}^{n} / k_{n} \rightarrow 0$ for all $m$ with $T_{m}(\varepsilon) \leq t$. Hence (5.18) follows from (5.16) and (5.14), and we are finished.

5.2. Proof of Theorem 3.2. Here $X$ is still an arbitrary semimartingale, and as for the previous theorem we can assume by localization that (5.7) holds. We first prove (a), and we assume Hypothesis (SN- $q$ ) with $q=p$ for proving (3.3) and $q=p+2 r$ for proving (3.4), so (5.3) implies

$$
\mathbb{E}\left(V(\chi, g, q, 0)_{t}^{n}\right)+\mathbb{E}\left(V(\chi, g, 0, q / 2)_{t}^{n}\right) \leq \frac{K t}{\Delta_{n} k_{n}^{q / 2}} \leq K t k_{n}^{2-q / 2} .
$$

We deduce from (5.17) that, for all $\eta>0$,

$$
\left|V(Z, g, q, 0)_{t}^{n}-V(X, g, q, 0)_{t}^{n}\right| \leq \eta V(X, g, q, 0)_{t}^{n}+K_{q, \eta} V(\chi, g, q, 0)_{t}^{n}
$$

and thus (3.3) follows from (3.2) and (5.19). 
Next, Hölder's inequality yields, when $p, r>0$ with $p+2 r=q>2$,

$$
V(Z, g, p, r)_{t}^{n} \leq\left(V(Z, g, q, 0)_{t}^{n}\right)^{p / q}\left(V(Z, g, 0, q / 2)_{t}^{n}\right)^{2 r / q}
$$

By (3.3), applied with $q$ instead of $p$, we see that the sequence $k_{n}^{-1} V(Z, g, q, 0)_{t}^{n}$ is tight, so for (3.4) it is enough to show that the sequence $k_{n}^{q / 2-2} V(Z, g, 0, q / 2)_{t}^{n}$ is also tight. To see this we first deduce from $\left|g_{j}^{\prime n}\right| \leq K / k_{n}$ that

$$
\widehat{X}(g)_{i}^{n} \leq \frac{K}{k_{n}^{2}} \sum_{j=i}^{i+k_{n}-1}\left(\Delta_{j}^{n} X\right)^{2},
$$

implying by Hölder's inequality (recall $q>2$ ) that $\left(\widehat{X}(g)_{i}^{n}\right)^{q / 2} \leq \frac{K}{k_{n}^{1+q / 2}} \times$ $\sum_{j=i}^{i+k_{n}-1}\left|\Delta_{j}^{n} X\right|^{q}$, and hence by (3.1) the sequence $k_{n}^{q / 2} V(X, g, 0, q / 2)_{t}^{n}$ is tight. Second, (5.19) yields that the sequence $k_{n}^{q / 2-2} V(\chi, g, 0, q / 2)_{t}^{n}$ is tight, and (3.4) follows because $V(Z, g, 0, q / 2)_{t}^{n} \leq K_{q}\left(V(X, g, 0, q / 2)_{t}^{n}+V(\chi, g, 0, q\right.$ ) $\left.2)_{t}^{n}\right)$.

Now we turn to (b), so we assume (SN-2). The left-hand side of (3.5) can be written as

$$
\frac{1}{k_{n}} V(X, g, 2,0)_{t}^{n}+\frac{1}{k_{n}} \sum_{l=1}^{4} U(l)_{t}^{n},
$$

where

$$
U(l)_{t}^{n}= \begin{cases}2 \sum_{i=0}^{\left[t / \Delta_{n}\right]-k_{n}} \bar{X}(g)_{i}^{n} \bar{\chi}(g)_{i}^{n}, & \text { if } l=1, \\ -\sum_{i=0}^{\left[t / \Delta_{n}\right]-k_{n}} \sum_{j=1}^{k_{n}}\left(g_{j}^{\prime n}\right)^{2} \Delta_{i+j}^{n} X \Delta_{i+j}^{n} \chi, & \text { if } l=2, \\ -\frac{1}{2} V(X, g, 0,1)_{t}^{n}, & \text { if } l=3, \\ V(\chi, g, 2,0)_{t}^{n}-\frac{1}{2} V(\chi, g, 0,1)_{t}^{n}, & \text { if } l=4,\end{cases}
$$

and by (3.2) it is enough to prove that for $l=1,2,3,4$,

$$
\frac{1}{k_{n}} U(l)_{t}^{n} \stackrel{\mathbb{P}}{\longrightarrow} 0
$$

Equation (5.20) yields $\left|U(3)_{t}^{n}\right| \leq \frac{K}{k_{n}} \sum_{i=1}^{\left[t / \Delta_{n}\right]}\left(\Delta_{i}^{n} X\right)^{2}$, so (5.21) for $l=3$ follows from (3.1). Next, (2.5) implies $\mathbb{E}\left(U(l)_{t}^{n} \mid \mathcal{F}^{(0)}\right)=0$ for $l=1$, 2 ; hence (5.21), for $l=1,2$ will be implied by

$$
\mathbb{E}\left(\left(\frac{1}{k_{n}} U(l)_{t}^{n}\right)^{2} \mid \mathcal{F}^{(0)}\right) \stackrel{\mathbb{P}}{\longrightarrow} 0 .
$$


By (2.5) and (2.11) and (5.3), the variables $\left|\mathbb{E}\left(\bar{\chi}(g)_{i}^{n} \bar{\chi}(g)_{j}^{n} \mid \mathcal{F}^{(0)}\right)\right|$ vanish if $j \geq k_{n}$ and are smaller than $K / k_{n}$ otherwise, whereas the variables $\left|\mathbb{E}\left(\Delta_{i}^{n} \chi \Delta_{i+j}^{n} \chi \mid \mathcal{F}^{(0)}\right)\right|$ are bounded, and vanish if $j \geq 2$. Then we get

$$
\begin{aligned}
\mathbb{E}\left(\left(U(1)_{t}^{n}\right)^{2} \mid \mathcal{F}^{(0)}\right) & \leq \frac{K}{k_{n}} \sum_{i=0}^{\left[t / \Delta_{n}\right]-k_{n}} \sum_{j=1}^{k_{n}} \bar{X}(g)_{i}^{n} \bar{X}(g)_{i+j}^{n} \leq K V(X, g, 2,0)_{t}^{n}, \\
\mathbb{E}\left(\left(U(2)_{t}^{n}\right)^{2} \mid \mathcal{F}^{(0)}\right) & \leq \frac{K}{k_{n}^{4}} \sum_{i, i^{\prime \prime}=0}^{\left[t / \Delta_{n}\right]-k_{n}} \sum_{j, j^{\prime}=0}^{k_{n}-1}\left|\Delta_{i+j}^{n} X \Delta_{i^{\prime}+j^{\prime}}^{n} X\right| 1_{\left\{\left|i^{\prime}+j^{\prime}-i-j\right| \leq 2\right\}} \\
& \leq \frac{K}{k_{n}^{2}} \sum_{i=1}^{\left[t / \Delta_{n}\right]}\left(\Delta_{i}^{n} X\right)^{2}
\end{aligned}
$$

and (5.22) follows from (3.2) when $l=1$ and from (3.1) when $l=2$.

Finally, an easy calculation shows that $U(4)_{t}^{n}=U(5)_{t}^{n}+U(6)_{t}^{n}$ where

$$
\begin{aligned}
U(5)_{t}^{n} & =\sum_{i=0}^{\left[t / \Delta_{n}\right]} \chi_{i}^{n} \sum_{j=1}^{k_{n}} \alpha_{i j}^{n} \chi_{i+j}^{n}, \\
U(6)_{t}^{n} & =\sum_{i=0}^{k_{n}}\left(\alpha_{i}^{\prime n}\left(\chi_{i}^{n}\right)^{2}+\alpha_{i}^{\prime \prime n}\left(\chi_{i+\left[t / \Delta_{n}\right]-k_{n}}^{n}\right)^{2}\right)
\end{aligned}
$$

for some coefficients $\alpha_{i j}^{n}, \alpha_{i}^{\prime n}, \alpha_{i}^{\prime \prime n}$, all smaller than $K / k_{n}$. Then, obviously, $\mathbb{E}\left(\left|U(6)_{t}^{n}\right|\right) \leq K$ and $E\left(U(5)_{t}^{n}\right)=0$, and, since $\mathbb{E}\left(\chi_{i}^{n} \chi_{i+j}^{n} \chi_{i^{\prime}}^{n} \chi_{i^{\prime}+j^{\prime}}^{n}\right)$ vanishes unless $i=i^{\prime}$ and $j=j^{\prime}$ when $j, j^{\prime} \geq 1$, we also have $\mathbb{E}\left(\left(U(5)_{t}^{n}\right)^{2}\right) \leq K t / k_{n} \Delta_{n} \leq$ $K t k_{n}$. Then (5.21) and (5.22) hold for $l=6$ and $l=5$, respectively, and thus (5.21) finally holds for $l=4$.

5.3. A key lemma. In this section we prove a key result, useful for deriving the other LLNs when the process $X$ is continuous and for all CLTs. Before that, we prove Lemma 3.5.

Proof of Lemma 3.5. By virtue of (3.14) we have

$$
\mu_{p}(g ; \eta, \zeta)=\sum_{v=0}^{p / 2} m_{2 v}\left(\eta^{2} \bar{g}(2)\right)^{v}\left(\zeta^{2} \bar{g}^{\prime}(2)\right)^{p / 2-v} \sum_{r=0}^{p / 2-v} C_{p-2 r}^{2 v} \rho_{p, r} 2^{r} m_{p-2 r-2 v}
$$

By (3.7) the last sum above vanishes if $v<p / 2$ and equals 1 when $v=p / 2$, hence (3.16). Next, we put $a_{i}=\mu_{p}\left(g_{i} ; \eta, \zeta\right)$ and $U_{t}^{i}=\eta L\left(g_{i}\right)_{t}+\zeta L^{\prime}\left(g_{i}\right)_{t}$, and, for $T \geq 2$,

$$
V_{T}^{i}=\sum_{r=0}^{p / 2} \rho_{p, r}\left(2 \zeta^{2} \bar{g}_{i}^{\prime}(2)\right)^{r} \int_{0}^{T}\left|U_{t}^{i}\right|^{p-2 r} d t
$$


The process $\left(L\left(g_{i}\right), L^{\prime}\left(g_{i}\right)\right)$ is stationary, and hence $\mathbb{E}^{\prime}\left(V_{T}^{i}\right)=T a_{i}$ for some constant $a_{i}$. Moreover, the functions

$$
f_{i j}(s, t)=\sum_{r, r^{\prime}=0}^{p / 2} \rho_{p, r} \rho_{p, r^{\prime}}\left(2 \zeta^{2}{\overline{g_{i}}}^{\prime}(2)\right)^{r}\left(2 \zeta^{2}{\overline{g_{j}}}^{\prime}(2)\right)^{r^{\prime}} \mathbb{E}^{\prime}\left(\left|U_{s}^{i}\right|^{p-2 r}\left|U_{t}^{j}\right|^{p-2 r^{\prime}}\right)-a_{i} a_{j},
$$

satisfy $f_{i j}(s, t)=f_{i j}(s+u, t+u)$ and $f_{i j}(s, t)=0$ if $|s-t|>1$. Thus if $T>2$,

$$
\begin{aligned}
\operatorname{Cov}\left(V_{T}^{i}, V_{T}^{j}\right)= & \int_{[0, T]^{2}} f_{i j}(s, t) d s d t \\
= & \int_{0}^{1} d s \int_{0}^{s+1} f_{i j}(s, t) d t+\int_{T-1}^{T} d s \int_{s-1}^{T} f_{i j}(s, t) d t \\
& +\int_{1}^{T-1} d s \int_{s-1}^{s+1} f_{i j}(s, t) d t .
\end{aligned}
$$

Therefore $\frac{1}{T} \operatorname{Cov}\left(V_{T}^{i}, V_{T}^{j}\right)$ converges to $\int_{0}^{2} f_{i j}(1, u) d u$ as $T \rightarrow \infty$, and this limit equals $\bar{\mu}_{2 p}\left(g_{i}, g_{j} ; \eta, \zeta\right)$. Since the limit of a sequence of covariance matrices is symmetric nonnegative, we have the result.

Now, we come to the aforementioned key result which consists of proving the convergence we hinted at after the definition (3.12) of the processes $L(g)$ and $L^{\prime}(g)$. For a precise statement, we fix a sequence $i_{n}$ of integers, and we associate the following processes with $g$, an arbitrary function satisfying (2.7):

$$
\begin{aligned}
\bar{L}(g)_{t}^{n} & =\sqrt{k_{n}} \bar{W}(g)_{i_{n}+\left[k_{n} t\right]}^{n}, \quad \bar{L}^{\prime}(g)_{t}^{n}=\sqrt{k_{n}} \bar{\chi}(g)_{i_{n}+\left[k_{n} t\right]}^{n}, \\
\widehat{L}^{\prime}(g)_{t}^{n} & =k_{n} \widehat{\chi}(g)_{i_{n}+\left[k_{n} t\right]}^{n} .
\end{aligned}
$$

We do not mention the sequence $i_{n}$ in this notation, but those processes clearly depend on it. In view of the "approximation" (5.1), these processes (and in particular their conditional moments of various orders) will play a central role in the sequel.

We fix a family $\left(g_{l}\right)_{1 \leq l \leq d}$ of weight functions satisfying (2.7). We denote by $\bar{L}_{t}^{n}$ and $\bar{L}_{t}^{\prime n}$ and $\widehat{L}_{t}^{\prime n}$ the $d$-dimensional processes with respective components, $\bar{L}\left(g_{l}\right)_{t}^{n}$ and $\bar{L}^{\prime}\left(g_{l}\right)_{t}^{n}$ and $\widehat{L}^{\prime}\left(g_{l}\right)_{t}^{n}$. These processes can be considered as variables with values in the Skorokhod space $\mathbb{D}^{d}$ of all càdlàg functions from $\mathbb{R}_{+}$into $\mathbb{R}^{d}$. The processes $L_{t}$ and $L_{t}^{\prime}$ with components $L\left(g_{l}\right)_{t}$ and $L^{\prime}\left(g_{l}\right)_{t}$, defined by (3.12) with the same Wiener processes $W^{1}$ and $W^{2}$ for all components, are also $\mathbb{D}^{d}$-valued variables, and the probability on $\mathbb{D}^{2 d}=\mathbb{D}^{d} \times \mathbb{D}^{d}$ which is the law of the pair, $\left(L, L^{\prime}\right)$ is denoted by $R=R_{\left(g_{v}\right)}=R(d x, d y)$.

We also have a sequence $\left(f_{n}\right)$ of functions on $\mathbb{D}^{3 d}$, all depending on $w \in \mathbb{D}^{3 d}$ only through their restrictions to $[0, m+1]$ for some $m \geq 0$ and which satisfy 
the following property for some $q^{\prime} \geq 2$ [below, $x, y, z \in \mathbb{D}^{d}$, so $v=(x, y) \in \mathbb{D}^{2 d}$ and $(x, y, z)=(v, z) \in \mathbb{D}^{3 d}$, and the same for $x^{\prime}, y^{\prime}, z^{\prime}$ and $v^{\prime}$; moreover for any multidimensional Borel function $u$ on $\mathbb{R}_{+}$we put $u_{m, n}^{\star}=\|u(0)\|+\|u(m)\|+$ $\left.\frac{1}{k_{n}} \sum_{i=1}^{(m+1) k_{n}}\left\|u\left(i / k_{n}\right)\right\|\right]$ :

$$
\begin{aligned}
& \left|f_{n}(v, z)\right| \leq K\left(1+\left(v_{m, n}^{\star}\right)^{q^{\prime}}+\left(z_{m, n}^{\star}\right)^{q^{\prime} / 2}\right), \\
& \left|f_{n}(v, z)-f_{n}\left(v^{\prime}, z^{\prime}\right)\right| \\
& \leq K\left(\left(v-v^{\prime}\right)_{m, n}^{\star}+\left(z-z^{\prime}\right)_{m, n}^{*}\right) \\
& \left.\times\left(1+\left(v_{m, n}^{\star}\right)^{q^{\prime}-1}+\left(v_{m, n}^{\prime \star}\right)^{q^{\prime}-1}+\left(z_{m, n}^{\star}\right)^{q^{\prime} / 2-1}+\left(z_{m, n}^{\prime \star}\right)^{q^{\prime} / 2-1}\right) .\right)
\end{aligned}
$$

Lemma 5.1. Assume Hypothesis $(S N-q)$ for some $q>4$ and that $\sigma$ is bounded. Let $\Gamma$ be the set of all times $s \geq 0$ such that both $\sigma$ and $\alpha$ are almost surely continuous at time $s$. Let $z_{0} \in \mathbb{D}^{d}$ be the constant function with components $\left(\bar{g}_{l}^{\prime}(2)\right)_{1 \leq l \leq d}$. Take any sequence $\left(i_{n}\right)$ of integers such that $s_{n}=i_{n} \Delta_{n}$ converges to some $s \in \Gamma$. If the sequence $\left(f_{n}\right)$ satisfies (5.24) for some $q^{\prime}<q$ and converges pointwise to a limit $f$, we have the almost sure convergence

$$
\mathbb{E}\left(f_{n}\left(\sigma_{s_{n}} \bar{L}^{n}, \bar{L}^{\prime n}, \widehat{L}^{\prime n}\right) \mid \mathcal{F}_{s_{n}}\right) \rightarrow \int f\left(\theta \sigma_{s} x, \alpha_{s} y, 2\left(\alpha_{s}\right)^{2} z_{0}\right) R(d x, d y) .
$$

Proof. (1) We first prove an auxiliary result. Let $\Omega_{s}^{(0)}$ be the set of all $\omega^{(0)}$ such that both $\sigma\left(\omega^{(0)}\right)$ and $\alpha\left(\omega^{(0)}\right)$ are continuous at time $s$. We have $\mathbb{P}^{(0)}\left(\Omega_{s}^{(0)}\right)=1$ because $s \in \Gamma$, and we fix $\omega^{(0)} \in \Omega_{s}^{(0)}$. We consider the probability space $\left(\Omega^{(1)}, \mathcal{F}^{(1)}, \mathbb{Q}\right)$ where $\mathbb{Q}=\mathbb{Q}\left(\omega^{(0)}, \cdot\right)$, and our aim in this step is to show that under $\mathbb{Q}$,

$$
\bar{L}^{\prime n} \stackrel{\mathcal{L}}{\longrightarrow} \alpha_{s}\left(\omega^{(0)}\right) L^{\prime}, \quad \mathbb{E}_{\mathbb{Q}}\left(\left(\bar{L}^{\prime n}\right)_{m, n}^{* q}\right) \leq K_{m}
$$

(functional convergence in law in $\mathbb{D}^{d}$ ). In view of the definition of $\left(\bar{L}^{\prime n}\right)_{m, n}^{*}$ (which is the norm of $t \rightarrow \bar{L}_{t}^{\prime n}$ described above), the second property immediately follows from (5.3).

We first prove the finite-dimensional convergence. Let $0<t_{1}<\cdots<t_{r}$. By (5.23) and (2.11) the $r d$-dimensional variable $Z_{n}=\left(\bar{L}_{t_{i}}^{\prime n, l}: 1 \leq l \leq d, 1 \leq i \leq r\right)$ is

$$
\begin{aligned}
& Z_{n}=\sum_{j=1}^{\infty} z_{j}^{n}, \quad \text { where } z_{j}^{n}=\zeta_{j}^{n} a_{j}^{n}, \zeta_{j}^{n}=\frac{1}{\sqrt{k_{n}}} \chi_{i_{n}+j-1}^{n} \quad \text { and } \\
& a_{j}^{n, l, i}=\left\{\begin{array}{ll}
-k_{n}\left(g_{l}\right)_{j-\left[k_{n} t_{i}\right]}^{\prime n}, & \text { if } 1+\left[k_{n} t_{i}\right] \leq j \leq k_{n}+\left[k_{n} t_{i}\right], \\
0, & \text { otherwise. }
\end{array}\right\}
\end{aligned}
$$

Under $\mathbb{Q}$ the variables $\zeta_{j}^{n}$ are independent centered, with $\mathbb{E}_{Q}\left(\left|\zeta_{j}^{n}\right|^{4}\right) \leq K k_{n}^{-2}$ by Hypothesis $(\mathrm{SN}-q)$; recall $q>4$. The numbers $a_{j}^{n, l, i}$ being uniformly 
bounded and equal to 0 when $j>k_{n}+\left[k_{n} t r\right]$, we deduce that under $\mathbb{Q}$ again the variables $z_{j}^{n}$ are independent with

$$
\mathbb{E}_{Q}\left(z_{j}^{n}\right)=0, \quad \mathbb{E}_{Q}\left(\left\|z_{j}^{n}\right\|^{4}\right) \leq K k_{n}^{-2}, \quad \sum_{j=1}^{\infty} \mathbb{E}_{Q}\left(\left\|z_{j}^{n}\right\|^{4}\right) \rightarrow 0 .
$$

Next,

$$
\sum_{j=1}^{\infty} \mathbb{E}_{Q}\left(z_{j}^{n, l, i} z_{j}^{n, l^{\prime}, i^{\prime}}\right)=\frac{1}{k_{n}} \sum_{j=1}^{\infty} \alpha_{\left(i_{n}+j-1\right) \Delta_{n}}\left(\omega^{(0)}\right)^{2} a_{j}^{n, l, i} a_{j}^{n, l^{\prime}, i^{\prime}} .
$$

On one hand $\alpha_{\left(i_{n}+j-1\right) \Delta_{n}}\left(\omega^{(0)}\right)^{2}$ converges uniformly in $j \leq k_{n}+\left[t_{r} k_{n}\right]$ to $\alpha_{s}\left(\omega^{(0)}\right)^{2}$ because $s \mapsto \alpha_{s}\left(\omega^{(0)}\right)$ is continuous at $s$. On the other hand, since $g_{l}=0$ outside $[0,1]$,

$$
\begin{aligned}
& \frac{1}{k_{n}} \sum_{j=1}^{\infty} a_{j}^{n, l, i} a_{j}^{n, l^{\prime}, i^{\prime}} \\
& \quad=k_{n} \sum_{j=1}^{\infty} \int_{(j-1) / k_{n}}^{j / k_{n}} g_{l}^{\prime}\left(u-\frac{\left[k_{n} t_{i}\right]}{k_{n}}\right) d u \int_{(j-1) / k_{n}}^{j / k_{n}} g_{l^{\prime}}^{\prime}\left(u-\frac{\left[k_{n} t_{i^{\prime}}\right]}{k_{n}}\right) d u,
\end{aligned}
$$

which clearly converges to $c^{l, i, l^{\prime}, i^{\prime}}=\int g_{l}^{\prime}\left(v-t_{i}\right) g_{l^{\prime}}^{\prime}\left(v-t_{i^{\prime}}\right) d v$ by the mean value theorem, the piecewise continuity of each $g_{l}^{\prime}$ and Riemann approximation. Hence

$$
\sum_{j=1}^{\infty} \mathbb{E}_{Q}\left(z_{j}^{n, l, i} z_{j}^{n, l^{\prime}, i^{\prime}}\right) \rightarrow c^{l, i, l^{\prime}, i^{\prime}} \alpha_{s}\left(\omega^{(0)}\right)^{2} .
$$

Then a standard limit theorem on row-wise independent triangular arrays of infinitesimal variables yield that $Z_{n}$ converges in law under $\mathbb{Q}$ to a centered Gaussian variable with covariance matrix $\left(c^{l, i, l^{\prime}, i^{\prime}} \alpha_{s}\left(\omega^{(0)}\right)^{2}\right)$ (see, e.g., Theorem VII-2-36 of [16]). Now, in view of (3.12), this matrix is the covariance of the centered Gaussian vector $\left(L_{t_{i}}^{\prime, l}: 1 \leq l \leq d, 1 \leq i \leq q\right)$, and the finite-dimensional convergence in (5.26) is proved.

To obtain the first property in (5.26) it remains to prove that for each $l$ the sequence of processes $\bar{L}^{\prime}\left(g_{l}\right)^{n}$ is C-tight. Equivalently, we can prove that the sequence of processes $G^{n}$ is C-tight, where $G^{n}$ is continuous, coincides with $\bar{L}^{\prime}\left(g_{l}\right)^{n}$ at all times $i / k_{n}$ and is piecewise linear between these times. For this we use a criterion given in [12] for example. Namely, since $q>2$, the C-tightness of the sequence $G^{n}$ is implied by

$$
0 \leq v \leq 1 \quad \Rightarrow \quad \mathbb{E}_{\mathbb{Q}}\left(\left|G_{t+v}^{n}-G_{t}^{n}\right|^{q}\right) \leq K v^{q / 2} .
$$

A simple computation shows that $G_{t+v}^{n}-G_{t}^{n}=\sum_{j} \delta_{j}^{n} \chi_{j}^{n}$ for suitable coefficients $\delta_{j}^{n}$, such that at most $2\left[k_{n} v\right]$ are smaller that $K_{1} / \sqrt{k_{n}}$, and at most 
$k_{n}$ of them are smaller than $K_{2} v / \sqrt{k_{n}}$, and all others vanish. Then the Burkholder-Davis-Gundy inequality yields

$$
\begin{aligned}
\mathbb{E}_{\mathbb{Q}}\left(\left|G_{t+v}^{n}-G_{t}^{n}\right|^{q}\right) & \leq K \mathbb{E}_{\mathbb{Q}}\left(\left(\sum_{j}\left(\delta_{j}^{n} \chi_{j}^{n}\right)^{2}\right)^{q / 2}\right) \\
& \leq K \beta(q)\left(\omega^{(0)}\right)\left(K_{1}^{q}(2 v)^{q / 2}+K_{2}^{q} v^{q}\right),
\end{aligned}
$$

and (5.29) follows. Then (5.26) is completely proved.

(2) In exactly the same setting as in the previous step, we prove here that

$$
\widehat{L}^{\prime n} \stackrel{\text { u.c.p. }}{\longrightarrow} 2\left(\alpha_{s}\left(\omega^{(0)}\right)\right)^{2} z_{0}, \quad \mathbb{E}_{\mathbb{Q}}\left(\sup _{v \leq t}\left\|\widehat{L}_{v}^{\prime n}\right\|^{q / 2}\right) \leq K_{t}
$$

(under $\mathbb{Q}$ again, and with $z_{0}$ as in the statement of the lemma). These are componentwise properties, so we may assume $d=1$ here and $g_{1}=g$. The second property again follows from (5.3). For the first one, we see that under $\mathbb{Q}$ the variable $\zeta_{t, j}^{n}=k_{n}\left(g^{\prime}\left(j / k_{n}\right) \Delta_{i_{n}+\left[k_{n} t\right]+j}^{n} \chi\right)^{2}$ satisfies

$$
\begin{aligned}
a_{t, j}^{n}: & =\mathbb{E}_{\mathbb{Q}}\left(\zeta_{t, j}^{n}\right) \\
& =k_{n}\left(g^{\prime}\left(j / k_{n}\right)\right)^{2}\left(\left(\alpha\left(\omega^{(0)}\right)_{\left(i_{n}+\left[k_{n} t\right]+j\right) \Delta_{n}}\right)^{2}\right. \\
& \left.+\left(\alpha\left(\omega^{(0)}\right)_{\left(i_{n}+\left[k_{n} t\right]+j-1\right) \Delta_{n}}\right)^{2}\right), \\
\mathbb{E}_{\mathbb{Q}}\left(\left|\zeta_{t, j}^{n}\right|^{q / 2}\right) & \leq K / k_{n}^{q / 2} .
\end{aligned}
$$

In view of the continuity of $\alpha\left(\omega^{(0)}\right)$ at time $s$ and of $(2.10)$, and since $\widehat{L}_{t}^{\prime n}=$ $\sum_{j=1}^{k_{n}} \zeta_{t, j}^{n}$, we see that $B_{t}^{n}=\mathbb{E}_{\mathbb{Q}}\left(\widehat{L}_{t}^{\prime n}\right)=\sum_{j=1}^{k_{n}} a_{t, j}^{n}$ converges locally uniformly to the "constant" $2\left(\alpha_{s}\left(\omega^{(0)}\right)\right)^{2} z_{0}$. Hence it remains to prove that $V_{t}^{n}=\widehat{L}_{t}^{\prime n}-$ $B_{t}^{n} \stackrel{\text { u.c.p. }}{\longrightarrow} 0$. For this it suffices to show $V_{t}^{n} \stackrel{\mathbb{P}}{\longrightarrow} 0$ for each $t$, and the Ctightness of both sequences $\left(\widehat{L}^{n}\right)$ and $\left(B^{n}\right)$, and the latter follows from $B_{t}^{n} \stackrel{\text { u.c.p. }}{\longrightarrow} 2\left(\alpha_{s}\left(\omega^{(0)}\right)\right)^{2} z_{0}$.

Now, $V_{t}^{n}$ is the sum of the $k_{n}$ centered variables $\zeta_{t, j}^{n}-a_{t, j}^{n}$, with $(q / 2)$ th absolute moment smaller than $K / k_{n}^{q / 2}$, and $\zeta_{t, j}^{n}$ is independent of $\left(\zeta_{t, l}^{n}: \mid l-\right.$ $j \mid \geq 2)$. Then obviously $\mathbb{E}_{Q}\left(\left(V_{t}^{n}\right)^{2}\right) \leq K / k_{n} \rightarrow 0$. For the C-tightness of $\left(\widehat{L}^{n}\right)$ it suffices as in the end of Step 1 to prove the C-tightness of the linearized versions $\left(G^{\prime n}\right)$ of $\left(\widehat{L}^{\prime n}\right)$. We have $G_{t+v}^{\prime n}-G_{t}^{\prime n}=\sum_{i} \delta_{i}^{n}\left(\Delta_{i}^{n} \chi\right)^{2}$ for suitable coefficients $\delta_{j}^{n}$, such that at most $2\left[k_{n} v\right]$ are smaller that $K_{1} / k_{n}$, and at most $k_{n}$ of them are smaller than $K_{2} v / k_{n}$, and all others vanish. Then by the Burkholder-Davis-Gundy inequality (applied separately for the sum of even indices and the sum of odd indices, to ensure the independence of the summands), we have

$$
\mathbb{E}_{\mathbb{Q}}\left(\left|G_{t+v}^{\prime n}-G_{t}^{\prime n}\right|^{q / 2}\right) \leq K \mathbb{E}_{\mathbb{Q}}\left(\left(\sum_{j}\left(\delta_{j}^{n} \chi_{j}^{n}\right)^{2}\right)^{q / 4}\right) \leq K v^{q / 4} .
$$


Since $q>4$, the C-tightness of $\left(G^{\prime n}\right)$ follows as in Step 1, and (5.30) holds.

(3) Now we draw some consequences of the previous facts. We set for $y, z \in \mathbb{D}^{d}$,

$$
\begin{aligned}
f_{\omega^{(0)}}^{n}(y, z) & =f_{n}\left(\sigma_{s_{n}}\left(\omega^{(0)}\right) L^{n}\left(\omega^{(0)}\right), y, z\right), \\
A_{j}^{n}\left(\omega^{(0)}\right) & = \begin{cases}\int \mathbb{Q}\left(\omega^{(0)}, d \omega^{(1)}\right) f_{\omega^{(0)}}^{n}\left(\bar{L}^{\prime n}\left(\omega^{(1)}\right), \widehat{L}^{\prime n}\left(\omega^{(1)}\right)\right), & j=1, \\
\int f_{\omega^{(0)}}^{n}\left(\alpha_{s_{n}}\left(\omega^{(0)}\right) y, 2 \alpha_{s}^{2} z_{0}\right) R(d x, d y), & j=2 .\end{cases}
\end{aligned}
$$

The $\mathcal{F}^{(0)}$-measurable variables,

$$
\Phi_{n}=1+\sup _{v \in\left[0,(m+1) u_{n}\right]} \sqrt{k_{n}}\left|W_{s_{n}+v}-W_{s_{n}}\right|,
$$

satisfy $\mathbb{E}\left(\Phi_{n}^{u}\right) \leq K_{u}$ for any $u>0$, by scaling of the Brownian motion $W$ whereas $\left\|\bar{L}_{t}^{n}\right\| \leq K \Phi_{n}$ if $t \leq m$. Then we deduce from (5.24) and from the boundedness of $\sigma$ and $\alpha$ that if $y, y^{\prime}, z, z^{\prime}$ are in $\mathbb{D}^{d}$ and $u=(y, z)$ and $u^{\prime}=$ $\left(y^{\prime}, z^{\prime}\right)$.

$$
\left.\begin{array}{r}
\begin{array}{l}
f_{\omega(0)}^{n}(u) \mid \leq K \Phi_{n}\left(\omega^{(0)}\right)^{q^{\prime}}\left(1+\left(y_{m, n}^{\star}\right)^{q^{\prime}}+\left(z_{m, n}^{\star}\right)^{q^{\prime} / 2}\right), \\
\left|f_{\omega^{(0)}}^{n}(u)-f_{\omega^{(0)}}^{n}\left(u^{\prime}\right)\right| \leq K \Phi_{n}\left(\omega^{(0)}\right)^{q^{\prime}}\left(u-u^{\prime}\right)_{m, n}^{\star}\left(1+\left(y_{m, n}^{\star}\right)^{q^{\prime}-1}+\left(y_{m, n}^{\prime \star}\right)^{q^{\prime}-1}\right. \\
\left.+\left(z_{m, n}^{\star}\right)^{q^{\prime} / 2-1}+\left(z_{m, n}^{\prime \star}\right)^{q^{\prime} / 2-1}\right) .
\end{array}
\end{array}\right\}
$$

Moreover $\alpha_{s_{n}}\left(\omega^{(0)}\right) \rightarrow \alpha_{s}\left(\omega^{(0)}\right)$, so by the Skorokhod representation theorem according to which, in case of convergence in law, one can replace the original variables by variables having the same laws and converging pointwise, one deduces from (5.26) and (5.30) [these imply that the variables $f_{\omega(0)}^{n}\left(\bar{L}^{\prime n}, \widehat{L}^{\prime n}\right)$ are uniformly integrable, since $\left.q^{\prime}<q\right]$, that

$$
\left.\begin{array}{l}
\omega^{(0)} \in \Omega_{s}^{(0)} \Rightarrow A_{1}^{n}\left(\omega^{(0)}\right)-A_{2}^{n}\left(\omega^{(0)}\right) \rightarrow 0, \\
\mathbb{E}\left(\left|A_{j}^{n}\right|^{q / q^{\prime}}\right) \leq K .
\end{array}\right\}
$$

Next, we make the following observation: due to the $\mathcal{F}^{(0)}$-conditional independence of the $\chi_{t}$ 's, a version of the conditional expectation in (5.25) is $\mathbb{E}\left(A_{1}^{n} \mid \mathcal{F}_{s_{n}}\right)$. Therefore in view of (5.31) (which ensures the uniform integrability and the a.s. convergence to 0 of the sequence $\left.A_{1}^{n}-A_{2}^{n}\right),(5.25)$ is implied by

$$
\mathbb{E}\left(A_{2}^{n} \mid \mathcal{F}_{s_{n}}\right) \rightarrow F\left(\sigma_{s}, \alpha_{s}\right) \quad \text { a.s. }
$$

where

$$
F(\eta, \zeta)=\int f\left(\theta \eta x, \zeta y, 2(\zeta)^{2} z_{0}\right) R(d x, d y)
$$


(4) For proving (5.32) we start again with an auxiliary result, namely

$$
\bar{L}^{n} \stackrel{\mathcal{L}}{\longrightarrow} \theta L .
$$

For this, we see that $Z_{n}=\left(\bar{L}_{t_{i}}^{n, l}: 1 \leq l \leq d, 1 \leq i \leq r\right)$ is given by $(5.27)$, except that

$\zeta_{j}^{n}=\sqrt{k_{n}} \Delta_{i_{n}+j}^{n} W, \quad a_{j}^{n, l, i}= \begin{cases}\left(g_{l}\right)_{j-\left[k_{n} t_{i}\right]}^{n}, & \text { if } 1+\left[k_{n} t_{i}\right] \leq j \leq k_{n}+\left[k_{n} t_{i}\right], \\ 0, & \text { otherwise. }\end{cases}$

Then the proof of (5.33), both for the finite-dimensional convergence and the C-tightness, is exactly the same as for (5.26) [note that the right-hand side of (5.28) is now $\theta^{2} \int g_{l}\left(v-t_{i}\right) g_{l^{\prime}}\left(v-t_{i^{\prime}}\right) d v$ which is the covariance matrix of $\left.\left(\theta L_{t_{i}}^{l}: 1 \leq l \leq d, 1 \leq i \leq r\right)\right]$. Further, since $\left\|\bar{L}_{t}^{n}\right\| \leq K \Phi_{n}$ if $t \leq m$,

$$
\mathbb{E}\left(\sup _{v \leq t}\left\|\bar{L}_{v}^{n}\right\|^{q}\right) \leq K_{t}
$$

(5) Now we introduce some functions on $\mathbb{R}^{2}$ :

$$
\begin{aligned}
& F_{n}(\eta, \zeta)=\int \mathbb{E}\left(f_{n}\left(\eta \bar{L}^{n}, \zeta y, 2(\zeta)^{2} z_{0}\right)\right) R(d x, d y), \\
& F_{n}^{\prime}(\eta, \zeta)=\int \mathbb{E}\left(f_{n}\left(\theta \eta L, \zeta y, 2(\zeta)^{2} z_{0}\right)\right) R(d x, d y) .
\end{aligned}
$$

Under $R$ the canonical process is locally in time bounded in each $\mathbb{L}^{r}$. Then in view of (5.24) we deduce from (5.33) and (5.34), and exactly as for (5.31), that $F_{n}-F_{n}^{\prime} \rightarrow 0$ locally uniformly in $\mathbb{R}^{2}$. We also deduce from (5.24) that $F_{n}^{\prime}\left(\eta_{n}, \zeta_{n}\right)-F_{n}^{\prime}(\eta, \zeta) \rightarrow 0$ whenever $\left(\eta_{n}, \zeta_{n}\right) \rightarrow(\eta, \zeta)$, and also that $F_{n}^{\prime} \rightarrow F$ pointwise because $f_{n} \rightarrow f$ pointwise, and hence we have $F_{n}\left(\eta_{n}, \zeta_{n}\right) \rightarrow F(\eta, \zeta)$.

At this point it remains to observe that, because $\left(W_{s_{n}+t}-W_{s_{n}}\right)_{t \geq 0}$ is independent of $\mathcal{F}_{s_{n}}$, we have $\mathbb{E}\left(A_{2}^{n} \mid \mathcal{F}_{s_{n}}\right)=F_{n}\left(\sigma_{s_{n}}, \alpha_{s_{n}}\right)$. Since $\left(\sigma_{s_{n}}, \alpha_{s_{n}}\right) \rightarrow$ $\left(\sigma_{s}, \alpha_{s}\right)$ a.s., we readily deduce $(5.25)$, and we are done.

REMARK 5.2. In the previous lemma, suppose that all $f_{n}$ (hence $f$ as well) only depend on $(x, y)$ and not on $z$; that is, the processes $\widehat{L}^{\prime n}$ do not enter the picture. Then it is easily seen from the previous proof that we do not need $q>4$, but only $q>2$.

5.4. Asymptotically negligible arrays. An array $\left(\delta_{i}^{n}\right)$ of nonnegative variables is called AN (for "asymptotically negligible") if

$$
\sqrt{\Delta_{n}} \sup _{0 \leq j \leq k_{n}} \mathbb{E}\left(\sum_{i=0}^{\left[t / u_{n}\right]} \delta_{i k_{n}+j}^{n}\right) \rightarrow 0, \quad\left|\delta_{i}^{n}\right| \leq K,
$$


for all $t>0$. With any process $\gamma$ (in the sequel, $\gamma$ will usually be $\gamma=\sigma$ or $\gamma=\alpha$ ) and any integer $m$ we associate the variables

$\Gamma(\gamma, m)_{i}^{n}=\sup _{t \in\left[i \Delta_{n}, i \Delta_{n}+(m+1) u_{n}\right]}\left|\gamma_{t}-\gamma_{i \Delta_{n}}\right|, \quad \Gamma^{\prime}(\gamma, m)_{i}^{n}=\mathbb{E}\left(\Gamma(\gamma, m)_{i}^{n} \mid \mathcal{F}_{i}^{n}\right)$.

Lemma 5.3. (a) If $\left(\delta_{i}^{n}\right)$ is an $A N$ array, we have

$$
\Delta_{n} \mathbb{E}\left(\sum_{i=1}^{\left[t / \Delta_{n}\right]} \delta_{i}^{n}\right) \rightarrow 0
$$

for all $t>0$, and the array $\left(\left(\delta_{i}^{n}\right)^{q}\right)$ is also $A N$ for each $q>0$.

(b) If $\gamma$ is a càdlàg bounded process, then for all $m \geq 1$ the two arrays $\left(\Gamma(\gamma, m)_{i}^{n}\right)$ and $\left(\Gamma^{\prime}(\gamma, m)_{i}^{n}\right)$ are $A N$.

Proof. (a) The left-hand side of (5.36) is smaller than a constant times the left-hand side of (5.35), hence the first claim. The second claim follows from Hölder's inequality if $q<1$, and from $\sum_{i \in I}\left(\delta_{i}^{n}\right)^{q} \leq K \sum_{i \in I} \delta_{i}^{n}$ if $q>1$ (recall that $\left.\left|\delta_{i}^{n}\right| \leq K\right)$.

(b) Let $\delta_{i}^{n}=\Gamma(\gamma, m)_{i}^{n}$. If $\varepsilon>0$, denote by $N(\varepsilon)_{t}$ the number of jumps of $\gamma$ with size bigger than $\varepsilon$ on the interval $[0, t]$, and by $v(\varepsilon, t, \eta)$ the supremum of $\left|\gamma_{s}-\gamma_{r}\right|$ over all pairs $(r, s)$ with $s \leq r \leq s+\eta$ and $s \leq t$ and such that $N(\varepsilon)_{s}-N(\varepsilon)_{r}=0$. Since $\gamma$ is bounded,

$u_{n} \sup _{0 \leq j \leq k_{n}} \mathbb{E}\left(\sum_{i=0}^{\left[t / u_{n}\right]} \delta_{i k_{n}+j}^{n}\right) \leq \mathbb{E}\left(t v\left(\varepsilon, t+1,(m+1) u_{n}\right)+(K t) \wedge\left(K u_{n} N(\varepsilon)_{t+1}\right)\right)$

as soon as $(m+2) u_{n} \leq 1$. Since $\lim _{\sup _{n \rightarrow \infty}} v\left(\varepsilon, t+1,(m+1) u_{n}\right) \leq \varepsilon$, Fatou's lemma implies that the limsup of the left-hand side above is smaller than $K t \varepsilon$, so we have (5.35) because $\varepsilon$ is arbitrarily small. Since $\mathbb{E}\left(\Gamma^{\prime}(\gamma, m)_{i}^{n}\right)=$ $\mathbb{E}\left(\Gamma(\gamma, m)_{i}^{n}\right)$, the second claim follows.

5.5. Some estimates. Here we provide a number of estimates under the following assumption for some $q>2$ :

- we have (2.14) and Hypothesis $(\mathrm{SN}-q)$, and $b$ and $\sigma$ are bounded, and $\sigma$ and $\alpha$ are càdlàg.

This list of estimates is quite tedious, but unfortunately they play a central role in many places in the sequel. We first introduce some notation where $i$ 
and $j$ are integers, $Y$ is an arbitrary process and $\rho_{p, l}$ is given by (3.7) and $i+j \geq 1$ in the first line below, and $p$ an even integer in (5.38):

$$
\left.\begin{array}{c}
\kappa_{i, j}^{n}=\sigma_{i}^{n} \Delta_{i+j}^{n} W+\Delta_{i+j}^{n} \chi, \\
\lambda_{i, j}^{n}=\Delta_{i+j}^{n} Z-\kappa_{i, j}^{n}=\Delta_{i+j}^{n} X-\sigma_{i}^{n} \Delta_{i+j}^{n} W \\
\bar{\kappa}(g)_{i, j}^{n}=\sum_{l=1}^{k_{n}-1} g_{l}^{n} \kappa_{i, j+l}^{n}, \quad \bar{\lambda}(g)_{i, j}^{n}=\sum_{l=1}^{k_{n}-1} g_{l}^{n} \lambda_{i, j+l}^{n}, \\
\widehat{\lambda}(g)_{i, j}^{n}=\sum_{l=1}^{k_{n}}\left(g_{l}^{\prime n} \lambda_{i, j+l}^{n}\right)^{2}, \\
\phi(Y, g, p)_{i}^{n}=\sum_{l=0}^{p / 2} \rho_{p, l}\left(\bar{Y}(g)_{i}^{n}\right)^{p-2 l}\left(\widehat{Y}(g)_{i}^{n}\right)^{l}, \\
\phi(g, p)_{i, j}^{n}=\sum_{l=0}^{p / 2} \rho_{p, l}\left(\bar{\kappa}(g)_{i, j}^{n}\right)^{p-2 l}\left(\widehat{\chi}(g)_{i, j}^{n}\right)^{l} .
\end{array}\right\}
$$

Recalling (5.1), we see that $\bar{\kappa}(g)_{i, j}^{n}$ is an approximation of $\bar{Z}(g)_{i}^{n}$, and its asymptotic behavior is described in Lemma 5.1. Then $\phi(g, p)_{i, j}^{n}$ is an approximation of $\phi(Y, g, p)_{i}^{n}$ whereas by (3.9) we have

$$
\bar{V}(Y, g, p)_{t}^{n}=\sum_{i=0}^{\left[t / \Delta_{n}\right]-k_{n}} \phi(Y, g, p)_{i}^{n} .
$$

One of the aims of the estimates below is to prove that these approximations induce a negligible error.

In the forthcoming inequalities, we have $0 \leq j \leq m k_{n}$ where $m$ is a fixed integer. First, if we use (5.4) and the boundedness of $g$, and also (5.3), we obtain for $u>0$

$$
\begin{aligned}
& \mathbb{E}\left(\left|\bar{X}(g)_{i}^{n}\right|^{u}+\left|\bar{W}(g)_{i}^{n}\right|^{u} \mid \mathcal{F}_{i}^{n}\right) \leq K_{u} \Delta_{n}^{u / 4}, \\
& \left.\begin{array}{l}
\left.u=q \Rightarrow \mathbb{E}\left(\left|\bar{Z}(g)_{i}^{n}\right|^{u}+\left|\bar{\kappa}(g)_{i}^{n}\right|^{u} \mid \mathcal{F}_{i}^{n}\right) \leq K_{u} \Delta_{n}^{u / 4},\right\} \\
\lambda_{i, j}^{n}=\int_{(i+j-1) \Delta_{n}}^{(i+j) \Delta_{n}}\left(b_{s} d s+\left(\sigma_{s}-\sigma_{i}^{n}\right) d W_{s}\right), \\
\bar{\lambda}(g)_{i, j}^{n}=\int_{(i+j) \Delta_{n}}^{\left(i+j+k_{n}\right) \Delta_{n}} g_{n}\left(s-(i+j) \Delta_{n}\right)\left(b_{s} d s+\left(\sigma_{s}-\sigma_{i}^{n}\right) d W_{s}\right) .
\end{array}\right\}
\end{aligned}
$$

Hence we obtain for $u \geq 1$, and recalling that $\Gamma(\sigma, m)_{i}^{n} \leq K$,

$$
\left.\begin{array}{l}
\mathbb{E}\left(\left|\lambda_{i, j}^{n}\right|^{u} \mid \mathcal{F}_{i}^{n}\right) \leq K_{u} \Delta_{n}^{u / 2}\left(\Delta_{n}^{u / 2}+\Gamma^{\prime}(\sigma, m)_{i}^{n}\right), \\
\mathbb{E}\left(\left|\bar{\lambda}(g)_{i, j}^{n}\right|^{u} \mid \mathcal{F}_{i}^{n}\right) \leq K_{u} \Delta_{n}^{u / 4}\left(\Delta_{n}^{u / 4}+\Gamma^{\prime}(\sigma, m)_{i}^{n}\right) .
\end{array}\right\}
$$


If $u$ is an odd integer, (5.39), (5.41) and an expansion of $\left(\sigma_{i}^{n} \bar{W}(g)_{i}^{n}+\bar{\lambda}(g)_{i}^{n}\right)^{u}$ yield

$$
\begin{aligned}
& \mathbb{E}\left(\left(\bar{W}(g)_{i}^{n}\right)^{u} \mid \mathcal{F}_{i}^{n}\right)=0, \\
& \mathbb{E}\left(\left(\bar{X}(g)_{i}^{n}\right)^{u} \mid \mathcal{F}_{i}^{n}\right) \leq K_{u} \Delta_{n}^{u / 4}\left(\Delta_{n}^{1 / 4}+\sqrt{\Gamma^{\prime}(\sigma, m)_{i}^{n}}\right) .
\end{aligned}
$$

Next, using $\left|g_{i}^{\prime n}\right| \leq K / k_{n}$ and (5.4) and the first part of (5.41), plus Hölder's inequality and the definition of $\widehat{Y}(g)_{i}^{n}$, plus the obvious fact that $\mathbb{E}\left(\left|\kappa_{i, j}^{n}\right|^{u} \mid \mathcal{F}_{i}^{n}\right) \leq K_{u}$ if $u \leq q$, and after some calculations, we get for $u \geq 1$

$$
\left.\begin{array}{l}
\mathbb{E}\left(\left|\widehat{X}(g)_{i}^{n}\right|^{u}+\left|\widehat{W}(g)_{i}^{n}\right|^{u} \mid \mathcal{F}_{i}^{n}\right) \leq K_{u} \Delta_{n}^{3 u / 2}, \\
u \leq q / 2 \Rightarrow \mathbb{E}\left(\left|\widehat{Z}(g)_{i+j}^{n}\right|^{u}+\left|\widehat{\chi}(g)_{i+j}^{n}\right|^{u} \mid \mathcal{F}_{i}^{n}\right) \leq K_{u} \Delta_{n}^{u / 2}, \\
u \leq q \Rightarrow \mathbb{E}\left(\left|\widehat{Z}(g)_{i+j}^{n}-\widehat{\chi}(g)_{i+j}^{n}\right|^{u} \mid \mathcal{F}_{i}^{n}\right) \leq K_{u} \Delta_{n}^{u} .
\end{array}\right\}
$$

Then, if we combine (5.39), (5.41) and (5.43), and use again Hölder's inequality, we obtain for all reals $l, u \geq 1$ and $r \geq 0$,

$$
\left.\begin{array}{rl}
(l+2 r) u \leq & q \\
\Rightarrow \mathbb{E}(\mid & \left(\bar{Z}(g)_{i+j}^{n}\right)^{l}\left(\widehat{Z}(g)_{i+j}^{n}\right)^{r} \\
& \left.-\left(\bar{\kappa}(g)_{i, j}^{n}\right)^{l}\left(\widehat{\chi}(g)_{i, j}^{n}\right)^{r} \mid \mathcal{F}_{i}^{n}\right) \\
& \leq K_{u, l, r} \Delta_{n}^{u l / 4+u r / 2}\left(\Delta_{n}^{u / 4}+\left(\Gamma^{\prime}(\sigma, m)_{i}^{n}\right)^{1-u(l+2 r-1) / q}\right), \\
2 r u \leq q \quad & \Rightarrow \mathbb{E}\left(\left|\left(\widehat{Z}(g)_{i+j}^{n}\right)^{r}-\left(\widehat{\chi}(g)_{i, j}^{n}\right)^{r}\right|^{u} \mid \mathcal{F}_{i}^{n}\right) \leq K_{u, r} \Delta_{n}^{r u / 2+u / 2} .
\end{array}\right\}
$$

Finally, by (5.38), this readily gives for $p \geq 2$ an even integer and $u \geq 1$ a real, such that $p u \leq q$,

$$
\begin{aligned}
& \mathbb{E}\left(\left|\phi(Z, g, p)_{i+j}^{n}\right|^{u}+\left|\phi(g, p)_{i, j}^{n}\right|^{u} \mid \mathcal{F}_{i}^{n}\right) \\
& \quad \leq K_{u, p} \Delta_{n}^{p u / 4} \\
& \quad \mathbb{E}\left(\left|\phi(Z, g, p)_{i+j}^{n}-\phi(g, p)_{i, j}^{n}\right|^{u} \mid \mathcal{F}_{i}^{n}\right) \\
& \quad \leq K_{u, p} \Delta_{n}^{p u / 4}\left(\Delta_{n}^{u / 4}+\left(\Gamma^{\prime}(\sigma, m)_{i}^{n}\right)^{1-u(p-1) / q}\right) .
\end{aligned}
$$

5.6. Proof of Theorem 3.3. By localization we can and will assume (5.37). We set

$$
\begin{aligned}
& \mu_{i}^{n}=\Delta_{n}^{-p / 4}\left|\bar{Z}(g)_{i}^{n}\right|^{p}, \quad \zeta_{i}^{n}=\Delta_{n}^{-p / 4}\left|\bar{\kappa}(g)_{i, 0}^{n}\right|^{p}, \\
& \gamma_{t}=m_{p}\left|\theta \bar{g}(2) \sigma_{t}^{2}+\frac{\bar{g}^{\prime}(2)}{\theta} \alpha_{t}^{2}\right|^{p / 2} .
\end{aligned}
$$

The left-hand side of (3.6) is $\sum_{i=0}^{\left[t / \Delta_{n}\right]-k_{n}} \mu_{i}^{n}$ whereas we deduce from (5.44) with $r=0$ and Lemma 5.3 that $\Delta_{n} \sum_{i=0}^{\left[t / \Delta_{n}\right]-k_{n}}\left|\mu_{i}^{n}-\zeta_{i}^{n}\right| \stackrel{\text { u.c.p. }}{\longrightarrow} 0$. Then it 
remains to prove

$$
\Delta_{n} \sum_{i=0}^{\left[t / \Delta_{n}\right]-k_{n}} \zeta_{i}^{n} \stackrel{\text { u.c.p. }}{\longrightarrow} \int_{0}^{t} \gamma_{s} d s .
$$

Set $\zeta_{i}^{\prime n}=\mathbb{E}\left(\zeta_{i}^{n} \mid \mathcal{F}_{i}^{n}\right)$. By $(5.39), \mathbb{E}\left(\left(\zeta_{i}^{n}\right)^{2} \mid \mathcal{F}_{i}^{n}\right) \leq K$, and in particular $\zeta_{i}^{\prime n} \leq$ $K$. Moreover $\zeta_{i}^{n}$ is $\mathcal{F}_{i+k_{n}}^{n}$-measurable, hence $\mathbb{E}\left(\left(\zeta_{i}^{n}-\zeta_{i}^{\prime n}\right)\left(\zeta_{j}^{n}-\zeta_{j}^{\prime n}\right)\right)=0$ if $|j-i| \geq k_{n}$, and

$$
\begin{aligned}
\mathbb{E}\left(\left|\Delta_{n} \sum_{i=0}^{\left[t / \Delta_{n}\right]-k_{n}}\left(\zeta_{i}^{n}-\zeta_{i}^{\prime n}\right)\right|^{2}\right) & =\Delta_{n}^{2} \sum_{i, j=1}^{\left[t / \Delta_{n}\right]-k_{n}} \mathbb{E}\left(\left(\zeta_{i}^{n}-\zeta_{i}^{\prime n}\right)\left(\zeta_{j}^{n}-\zeta_{j}^{\prime n}\right)\right) \\
& \leq K \Delta_{n} k_{n} \rightarrow 0 .
\end{aligned}
$$

Thus it is enough to prove (5.46) with $\zeta_{i}^{n}$ substituted with $\zeta_{i}^{\prime n}$. Since $\gamma_{t}+$ $\zeta_{i}^{\prime n} \leq K$

$$
\left|\Delta_{n} \sum_{i=0}^{\left[t / \Delta_{n}\right]-k_{n}} \zeta_{i}^{\prime n}-\int_{0}^{t} \gamma_{s} d s\right| \leq \int_{\Delta_{n}}^{\left(\left[t / \Delta_{n}\right]-k_{n}\right) \Delta_{n}}\left|\gamma_{s}^{n}-\gamma_{s}\right| d s+K k_{n} \Delta_{n},
$$

where $\gamma_{s}^{n}=\zeta_{i}^{\prime n}$ when $(i-1) \Delta_{n} \leq s<i \Delta_{n}$. Therefore, since $\left|\gamma_{s}^{n}-\gamma_{s}\right| \leq K$, in order to obtain (5.46) it is enough to prove that for Lebesgue-almost all $s$ we have $\gamma_{s}^{n} \rightarrow \gamma_{s}$ a.s. In particular it is enough to prove that, for all $s \in \Gamma$ (cf. Lemma 5.1), we have

$$
\zeta_{\left[s / \Delta_{n}\right]+1}^{\prime n} \rightarrow \gamma_{s} \quad \text { a.s. }
$$

With the notation of Lemma 5.1, we take $d=1$ and the weight function $g_{1}=g$, and the functions $f_{n}=f$ on $\mathbb{D}^{3}$ as $f(x, y, z)=|x(0)+y(0)|^{p}$, so (5.24) is satisfied with $q^{\prime}=p<q$ and $m=0$. Moreover we fix $s \in \Gamma$ and set $i_{n}=\left[s / \Delta_{n}\right]+1$, so $s_{n}=i_{n} \Delta_{n} \rightarrow s$. The left-hand side of (5.25) is $\Delta_{n}^{p / 4} k_{n}^{p / 2} \zeta_{i_{n}}^{\prime n}$ whereas its right-hand side is $\mathbb{E}^{\prime}\left(\left|\theta \eta L(g)_{0}+\eta^{\prime} L^{\prime}(g)_{0}\right|^{p}\right)$ [recall (3.12)] evaluated at $\eta=\sigma_{s}$ and $\eta^{\prime}=\alpha_{s}$. Since $L(g)_{0}$ and $L^{\prime}(g)_{0}$ are independent centered normal with respective variances $\bar{g}(2)$ and $\bar{g}^{\prime}(2)$, this right-hand side is $m_{p}\left(\theta^{2} \bar{g}(2) \sigma_{s}^{2}+\bar{g}^{\prime}(2) \alpha_{s}^{2}\right)^{p / 2}=\theta^{p / 2} \gamma_{s}$. Since $\Delta_{n}^{p / 4} k_{n}^{p / 2} \rightarrow \theta^{p / 2}$, we get (5.47).

5.7. Proof of Theorem 3.4. As we said already, (a) is a particular case of (3.3) when $p \geq 4$, and of (3.5) when $p=2$. For (b), we can again assume (5.37). We set

$$
\begin{aligned}
\mu_{i}^{n} & =\Delta_{n}^{-p / 4} \phi(Z, g, p)_{i}^{n}, \quad \zeta_{i}^{n}=\Delta_{n}^{-p / 4} \phi(g, p)_{i, 0}^{n}, \\
\gamma_{t} & =m_{p}(\theta \bar{g}(2))^{p / 2}\left|\sigma_{t}\right|^{p},
\end{aligned}
$$

and $\zeta_{i}^{\prime n}=\mathbb{E}\left(\zeta_{i}^{n} \mid \mathcal{F}_{i}^{n}\right)$. We deduce from (5.45) and Lemma 5.3 that $\Delta_{n} \times$ $\sum_{i=0}^{\left[t / \Delta_{n}\right]-k_{n}}\left|\mu_{i}^{n}-\zeta_{i}^{n}\right| \stackrel{\text { u.c.p. }}{\longrightarrow} 0$. Then it is enough to prove (5.46). 
By (5.45) we have $\mathbb{E}\left(\left(\zeta_{i}^{n}\right)^{2} \mid \mathcal{F}_{i}^{n}\right) \leq K$; hence $\zeta_{i}^{\prime n} \leq K$. Then, exactly as in the previous proof, it remains to show (5.47) when $s \in \Gamma$. For this, we use Lemma 5.1 with $d=1$ and $g_{1}=g$ and the functions $f_{n}=f$ given by

$$
f(x, y, z)=\sum_{l=0}^{p / 2} \rho_{p, l}|x(0)+y(0)|^{p-2 l}|z(0)|^{l} .
$$

The left-hand side of (5.25) is again $\Delta_{n}^{p / 4} k_{n}^{p / 2} \zeta_{i_{n}}^{\prime n}$. Its right-hand side is $\mu_{p}(g$; $\left.\theta \sigma_{s}, \alpha_{s}\right)$, as given by (3.15), and by (3.16) this is also $\theta^{p / 2} \gamma_{s}$. Then (5.10) holds.

5.8. Proof of Theorem 3.6. The proof is basically the same as in the previous subsection, using again Lemma 5.1 and the fact that we deal with asymptotically $k_{n}$-dependent variables. We can assume (5.37), and we have

$$
\begin{aligned}
& \bar{\mu}_{2 p}(g, h ; \eta, \zeta) \\
& =\sum_{r, r^{\prime}=0}^{p / 2} \rho_{p, r} \rho_{p, r^{\prime}}\left(2 \zeta^{2} \bar{g}^{\prime}(2)\right)^{r}\left(2 \zeta^{2} \bar{h}^{\prime}(2)\right)^{r^{\prime}} \\
& \quad \quad \times\left(m_{p-2 r, p-2 r^{\prime}}(g, h ; \eta, \zeta)-2 m_{p-2 r}(g ; \eta, \zeta) m_{p-2 r^{\prime}}(h ; \eta, \zeta)\right) .
\end{aligned}
$$

Therefore is is enough to prove that for $r, r^{\prime}$ between 0 and $p / 2$, and with the notation

$$
\begin{gathered}
\mu_{i}^{n}=\Delta_{n}^{-p / 2}\left(\widehat{Z}(g)_{i}^{n}\right)^{r}\left(\widehat{Z}(h)_{i}^{n}\right)^{r^{\prime}}\left(\left|\bar{Z}(g)_{i+k_{n}}^{n}\right|^{p-2 r} \frac{1}{k_{n}} \sum_{j=1}^{2 k_{n}}\left|\bar{Z}(h)_{i+j}^{n}\right|^{p-2 r^{\prime}}\right. \\
\left.-2\left|\bar{Z}(g)_{i}^{n}\right|^{p-2 r}\left|\bar{Z}(h)_{i+k_{n}}^{n}\right|^{p-2 r^{\prime}}\right) \\
\gamma_{t}=\theta^{-p / 2}\left(2 \alpha_{t}^{2} \bar{g}^{\prime}(2)\right)^{r+r^{\prime}}\left(m_{p-2 r, p-2 r^{\prime}}\left(g, h ; \theta \sigma_{t}, \alpha_{t}\right)\right. \\
\left.-2 m_{p-2 r}\left(g ; \theta \sigma_{t}, \alpha_{t}\right) m_{p-2 r^{\prime}}\left(h ; \theta \sigma_{t}, \alpha_{t}\right)\right),
\end{gathered}
$$

we have

$$
\Delta_{n} \sum_{i=0}^{\left[t / \Delta_{n}\right]-3 k_{n}} \mu_{i}^{n} \stackrel{\text { u.c.p. }}{\longrightarrow} \int_{0}^{t} \gamma_{s} d s .
$$

By (5.43) and (5.44) we have $\Delta_{n} \sum_{i=0}^{\left[t / \Delta_{n}\right]-3 k_{n}}\left|\mu_{i}^{n}-\zeta_{i}^{n}\right| \stackrel{\text { u.c.p. }}{\longrightarrow} 0$ where

$$
\zeta_{i}^{n}=\Delta_{n}^{-p / 2}\left(\widehat{\chi}(g)_{i}^{n}\right)^{r}\left(\widehat{\chi}(h)_{i}^{n}\right)^{r^{\prime}}\left(\left|\bar{\kappa}(g)_{i, k_{n}}^{n}\right|^{p-2 r} \frac{1}{k_{n}} \sum_{j=1}^{2 k_{n}}\left|\bar{\kappa}(h)_{i, j}^{n}\right|^{p-2 r^{\prime}}\right.
$$




$$
\left.-2\left|\bar{\kappa}(g)_{i, 0}^{n}\right|^{p-2 r}\left|\bar{\kappa}(h)_{i, k_{n}}^{n}\right|^{p-2 r^{\prime}}\right),
$$

so we are left to prove

$$
\Delta_{n} \sum_{i=0}^{\left[t / \Delta_{n}\right]-3 k_{n}} \zeta_{i}^{n} \stackrel{\text { u.c.p. }}{\longrightarrow} \int_{0}^{t} \gamma_{s} d s .
$$

We set $\zeta_{i}^{\prime n}=\mathbb{E}\left(\zeta_{i}^{n} \mid \mathcal{F}_{i}^{n}\right)$, so as in the proof of Theorem 3.3 it is enough to prove (5.47) when $s \in \Gamma$. We apply Lemma 5.1 with $d=2$ and $g_{1}=g$ and $g_{2}=h$ and the functions $f_{n}$ and $f$ on $\mathbb{D}^{6}$ defined by

$$
\begin{aligned}
& f_{n}\left(\left(x, x^{\prime}\right),\left(y, y^{\prime}\right),\left(z, z^{\prime}\right)\right) \\
& =z(0)^{r} z^{\prime}(0)^{r^{\prime}}\left(|x(1)+y(1)|^{p-2 r} \frac{1}{k_{n}} \sum_{j=1}^{2 k_{n}}\left|x^{\prime}\left(\frac{j}{k_{n}}\right)+y^{\prime}\left(\frac{j}{k_{n}}\right)\right|^{p-2 r^{\prime}}\right. \\
& \left.\quad-2|x(0)+y(0)|^{p-2 r}\left|x^{\prime}(1)+y^{\prime}(1)\right|^{p-2 r^{\prime}}\right), \\
& \left.f\left(\left(x, x^{\prime}\right),\left(y, y^{\prime}\right),\left(z, z^{\prime}\right)\right) \quad-2|x(0)+y(0)|^{p-2 r}\left|x^{\prime}(1)+y^{\prime}(1)\right|^{p-2 r^{\prime}}\right) \\
& =z(0)^{r} z^{\prime}(0)^{r^{\prime}}\left(\left|x(1)+y^{\prime}(1)\right|^{p-2 r} \int_{0}^{2}\left|x^{\prime}(t)+y^{\prime}(t)\right|^{p-2 r^{\prime}} d t\right.
\end{aligned}
$$

and again $i_{n}=\left[s \Delta_{n}\right]+1$. Then (5.24) is satisfied with $q^{\prime}=2 p<q$ and $m=1$, and $f_{n} \rightarrow f$ pointwise. The left-hand side of (5.25) is $\Delta_{n}^{p / 2} k_{n}^{p} \zeta_{i_{n}}^{\prime n}$, whereas its right-hand side is $\theta^{p / 2} \gamma_{s}$ [recall that $\left(L(g)_{0}, L^{\prime}(g)_{0}\right)$ and $\left(L(h)_{1}, L^{\prime}(h)_{1}\right)$ are independent]. Since $\Delta_{n}^{p / 2} k_{n}^{p} \rightarrow \theta^{p / 2}$, we get (5.47) by the lemma, and the proof is finished.

5.9. Auxiliary results on the noise process. At this stage we start the proof of our CLTs, and this is done through a large number of steps. In the first, crucial step we derive some estimates on the (conditional) moments of the noise process $\chi$. Recall that $\mathcal{G}_{i}^{n}$ denotes the $\sigma$-field generated by $\mathcal{F}^{(0)}$ and $\mathcal{F}_{i}^{n}$. Set

$$
A(g)_{i}^{n}=\sum_{j=1}^{k_{n}}\left(g_{j}^{\prime n}\right)^{2}\left(\alpha_{i+j-1}^{n}\right)^{2} .
$$

For random variables $U_{\gamma}$ and $V_{\gamma}$ indexed by a parameter $\gamma$ [for example, $\gamma=(n, i)$ just below], with $V_{\gamma}>0$, we write $U_{\gamma}=\mathrm{O}_{u}\left(V_{\gamma}\right)$ if the family $U_{\gamma} / V_{\gamma}$ is bounded in probability. 
Lemma 5.4. Assume Hypothesis $(S N-q)$ for some $q \geq 2$, and let $v$ and $r$ be integers such that $2 \leq v+2 r \leq q$. Let also $m \geq 0$ and $j$ be arbitrary in $\left\{0,1, \ldots, m k_{n}\right\}$.

(a) When $v$ is even we have

$$
\begin{aligned}
& \mathbb{E}\left(\left(\bar{\chi}(g)_{i+j}^{n}\right)^{v}\left(\widehat{\chi}(g)_{i+j}^{n}\right)^{r} \mid \mathcal{G}_{i}^{n}\right) \\
& \quad=m_{v} 2^{r}\left(A(g)_{i+j}^{n}\right)^{r+v / 2}+\mathrm{O}_{u}\left(\Delta_{n}^{r / 2+v / 4+1 / 2}\right) \\
& \quad=m_{v} 2^{r} \frac{\bar{g}^{\prime}(2)^{r+v / 2}}{k_{n}^{r+v / 2}}\left(\alpha_{i}^{n}\right)^{2 r+v}+\mathrm{O}_{u}\left(\Delta_{n}^{r / 2+v / 4}\left(\Delta_{n}^{1 / 2}+\Gamma(\alpha, m)_{i}^{n}\right)\right) .
\end{aligned}
$$

(b) When $v$ is odd we have

$$
\mathbb{E}\left(\left(\bar{\chi}(g)_{i+j}^{n}\right)^{v}\left(\widehat{\chi}(g)_{i+j}^{n}\right)^{r} \mid \mathcal{G}_{i}^{n}\right)=\mathrm{O}_{u}\left(\Delta_{n}^{r / 2+v / 4+1 / 4}\right),
$$

and also, for some suitable numbers $\gamma_{v, r}$, depending on $g$,

$$
\begin{aligned}
\mathbb{E}\left(\left(\bar{\chi}(g)_{i+j}^{n}\right)^{v}\left(\widehat{\chi}(g)_{i+j}^{n}\right)^{r} \mid \mathcal{G}_{i}^{n}\right) & \\
= & \frac{\gamma_{v, r}}{k_{n}^{r+v / 2+1 / 2}}\left(\alpha_{i}^{n}\right)^{2 r+v-3} \beta(3)_{i}^{n} \\
& \quad+\mathrm{O}_{u}\left(\Delta_{n}^{r / 2+v / 4+1 / 4}\left(\Delta_{n}^{1 / 4}+\Gamma(\alpha, m)_{i}^{n}+\Gamma(\beta(3), m)_{i}^{n}\right)\right) .
\end{aligned}
$$

Proof. Equations (5.50) and (5.51) are simple consequences of (5.49) and (5.52), respectively, upon observing that $A(g)_{i+j}^{n}=\bar{g}^{\prime}(2)\left(\alpha_{i}^{n}\right)^{2} / k_{n}+$ $\mathrm{O}_{u}\left(\Delta_{n}^{1 / 2}\left(\Delta_{n}^{1 / 2}+\Gamma(\alpha, m)_{i}^{n}\right)\right)$. As for (5.49) and (5.52), and up to taking a further conditional expectation, it is enough to prove them when $j=0$, so in the rest of the proof we take $j=0$, and thus $m=0$ as well. The product $\left(\bar{\chi}(g)_{i}^{n}\right)^{v}\left(\widehat{\chi}(g)_{i}^{n}\right)^{r}$ is the sum of all the terms of the form

$$
\left.\begin{array}{rl}
\Phi(J, n)= & (-1)^{v} \prod_{l=1}^{v} g_{j_{l}}^{\prime n} \chi_{i+j_{l}-1}^{n} \prod_{l=1}^{s}\left(g_{j_{l}^{\prime}}^{\prime n} \chi_{i+j_{l}^{\prime}+\bar{j}_{l}^{\prime}-1}^{n}\right)^{2} \\
& \times \prod_{l=1}^{r-s}\left(-2\left(g_{j_{l}^{\prime \prime}}^{\prime n}\right)^{2} \chi_{i+j_{l}^{\prime \prime}}^{n} \chi_{i+j_{l}^{\prime \prime}-1}^{n}\right), \\
J= & \left\{s, j_{1}, \ldots, j_{v}, j_{1}^{\prime}, \ldots, j_{s}^{\prime}, \bar{j}_{1}^{\prime}, \ldots, \bar{j}_{s}^{\prime}, j_{1}^{\prime \prime}, \ldots, j_{r-s}^{\prime \prime}\right\}, \\
& \text { where } s \in\{0, \ldots, r\}, j_{l}, j_{l}^{\prime}, j_{l}^{\prime \prime} \in\left\{1, \ldots, k_{n}\right\}, \bar{j}_{l}^{\prime} \in\{0,1\} .
\end{array}\right\}
$$

We denote by $I(J)$ the family of all indices of the variables $\chi_{j}^{n}$ occurring in (5.53), the index $j$ appearing $l$ times if $\chi_{j}^{n}$ is taken at the power $l$, so that $I(J)$ contains $v+2 r$ indices. We also denote by $D(u)^{n}$ the class of all $J$ 's such that among the $v+2 r$ indices in $I(J)$, there are exactly $u$ different indices, each one appearing at least twice. Note that $D(u)^{n}$ is the disjoint union over $s^{\prime}=0, \ldots, r$ of the set $D\left(u, s^{\prime}\right)^{n}$ of all $J \in D(u)^{n}$ such that $s=s^{\prime}$. Note also that $D(u)^{n}=\varnothing$ if $u>v / 2+r$. 
By (2.5) and the $\mathcal{F}^{(0)}$-conditional independence of the $\chi_{t}$ 's, the conditional expectation $\mathbb{E}\left(\Phi(J, n) \mid \mathcal{G}_{i}^{n}\right)$ is always smaller than $K / k_{n}^{v+2 r}$, and it vanishes if $J$ is outside $\bigcup_{u \geq 1} D(u)^{n}$; that is,

$$
\mathbb{E}\left(\left(\bar{\chi}(g)_{i}^{n}\right)^{v}\left(\widehat{\chi}(g)_{i}^{n}\right)^{r} \mid \mathcal{G}_{i}^{n}\right)=\sum_{u=1}^{[v / 2]+r} \bar{\Phi}_{u}^{n},
$$

where

$$
\bar{\Phi}_{u}^{n}=\sum_{s=0}^{r} \bar{\Phi}(u, s)^{n}, \quad \bar{\Phi}(u, s)^{n}=\sum_{J \in D(u, s)^{n}} \mathbb{E}\left(\Phi(J, n) \mid \mathcal{G}_{i}^{n}\right) .
$$

Now $\# D(u, s)^{n} \leq K k_{n}^{u}$, so $\left|\bar{\Phi}(u, s)^{n}\right| \leq K k_{n}^{u-v-2 r}$; hence $\bar{\Phi}_{u}^{n}=\mathrm{O}_{u}\left(\Delta_{n}^{r / 2+v / 4+1 / 4}\right)$ as soon as $u \leq r-1 / 2+v / 2$. We deduce that for proving (5.49), so $v$ is even, it is enough to show that $\bar{\Phi}_{u}^{n}$ equals the right-hand side of (5.49), for $u=r+v / 2$. In the same way, for proving (5.52), so $v$ is odd, it is enough to show that $\bar{\Phi}_{u}^{n}$ equals the right-hand side of (5.52) for $u=r+(v-1) / 2$.

(a) Suppose that $v$ is even and $u=r+v / 2$. The definition of $D(u)^{n}$ and the property $u=r+v / 2$ yield that, if $J \in D(u)^{n}$, there is a nonnegative integer $w \leq \frac{v}{2} \wedge \frac{r-s}{2}$ such that $\Phi(J, n)$ is the product of $\frac{v+s+r-w}{2}$ terms, of three types, all for different indices for $\chi^{n}$ :

(1) $s-w+\frac{v}{2}$ terms of the form $\left(g_{j}^{\prime n} \chi_{i+j-1}^{n}\right)^{2}$ or $\left(g_{j}^{\prime n} \chi_{i+j}^{n}\right)^{2}$;

(2) $w$ terms of the form $-2\left(g_{j}^{\prime n}\right)^{3} g_{j+1}^{\prime n}\left(\chi_{i+j-1}^{n} \chi_{i+j}^{n}\right)^{2}$;

(3) $\frac{r-s-w}{2}$ terms of the form $4\left(g_{j}^{\prime n}\right)^{4}\left(\chi_{i+j-1}^{n} \chi_{i+j}^{n}\right)^{2}$.

Hence \#D(u,s) $\leq K k_{n}^{(v+s+r) / 2}$ because the number of terms for a particular $J$ is smaller than $\frac{v+s+r}{2}$, and the indices range from 1 to $k_{n}$. Moreover, since $\alpha$ is bounded and $\left|g_{j}^{\prime n}\right| \leq K / k_{n}$, we have $\mathbb{E}\left(|\Phi(J, n)| \mid \mathcal{G}_{i}^{n}\right) \leq K / k_{n}^{v+2 r}$. We then deduce that

$$
\left|\bar{\Phi}(u, s)^{n}\right| \leq K k_{n}^{(v+s+r) / 2-v-2 r} \leq K \Delta_{n}^{r / 2+v / 4+(r-s) / 2} .
$$

In particular, $\bar{\Phi}(u, s)^{n}=\mathrm{O}\left(\Delta_{n}^{r / 2+v / 4+1 / 2}\right)$ when $s<r$, and it thus remains to prove that $\bar{\Phi}(u, r)^{n}$ is equal to the right-hand side of (5.49). If $J \in D(u, r)^{n}$, then $\Phi(J, n)$ contains only terms of type (1). In fact $D(u, r)^{n}$ contains exactly the families $J$ for which $s=r$, and among $j_{1}, \ldots, j_{v}$ there are $v / 2$ distinct indices, each one appearing twice (we then denote by $J_{1}$ the set of the $v / 2$ distinct indices), and the sets $J_{2}=\left\{j_{l}^{\prime}+\bar{j}_{l}^{\prime}: 1 \leq l \leq r, \bar{j}_{l}^{\prime}=0\right\}$ and $J_{3}=\left\{j_{l}^{\prime}+\bar{j}_{l}^{\prime}: 1 \leq l \leq r, \bar{j}_{l}^{\prime}=1\right\}$ have distinct indices, and $J_{1}, J_{2}$ and $J_{3}$ are pair-wise disjoint. With this notation, we have (with $u$ terms all together in the products)

$$
\mathbb{E}\left(\Phi(J, n) \mid \mathcal{G}_{i}^{n}\right)=\prod_{j \in J_{1} \cup J_{2}}\left(g_{j}^{\prime n} \alpha_{i+j-1}^{n}\right)^{2} \prod_{j \in J_{3}}\left(g_{j-1}^{\prime n} \alpha_{i+j-1}^{n}\right)^{2} .
$$


The assumption (2.7) on $g$ yields that $\left|g_{j}^{\prime n}-g_{j-1}^{\prime n}\right| \leq K / k_{n}^{2}$, except for $j$ belonging to the set $Q_{n}$ of indices for which $g^{\prime}$ fails to exist or to be Lipschitz on $\left[(j-1) / k_{n}, j k_{n}\right]$, so $\# Q_{n} \leq K$. Since $\alpha_{i}^{n} \leq K$, we thus have

$$
\mathbb{E}\left(\Phi(J, n) \mid \mathcal{G}_{i}^{n}\right)= \begin{cases}\prod_{j \in J_{1} \cup J_{2} \cup J_{3}}\left(g_{j}^{\prime n} \alpha_{i+j-1}^{n}\right)^{2}+\mathrm{O}_{u}\left(k_{n}^{-2 u-1}\right), \\ \mathrm{O}_{u}\left(k_{n}^{-2 u}\right), & \text { if } Q_{n} \cap\left(J_{1} \cup J_{2} \cup J_{3}\right)=\varnothing,\end{cases}
$$

Consider now $L=\left\{l_{1}, \ldots, l_{u}\right\}$ in the set $\mathcal{L}_{n}$ of all families of indices with $1 \leq l_{1}<\cdots<l_{u} \leq k_{n}$, and let $w_{n}(L)$ be the number of $J \in D(u, r)^{n}$ such that

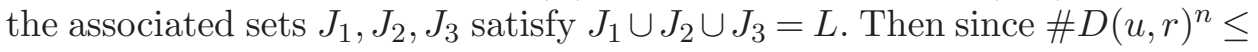
$K k_{n}^{u}$ and $\sup _{n} \# Q_{n}<\infty$, we deduce from the above that

$$
\bar{\Phi}(u, r)^{n}=\sum_{L \in \mathcal{L}_{n}} w_{n}(L) \prod_{j \in L}\left(g_{j}^{\prime n} \alpha_{i+j-1}^{n}\right)^{2}+\mathrm{O}_{u}\left(\Delta_{n}^{u / 2+1 / 2}\right) .
$$

Now we have to evaluate $w_{n}(L)$. There are $C_{u}^{r}$ many ways of choosing the two complementary subsets, $J_{1}$ and $J_{2} \cup J_{3}$, of $L$. Next, with $J_{1}$ given, there are $(v / 2) !(v-1)(v-3) \cdots 3 \cdot 1$ ways of choosing the indices $j_{l}$ so that $j_{1}, \ldots, j_{v}$ has $v / 2$ paired distinct indices which are the indices in $J_{1}$, and we recall that $(v-1)(v-3) \cdots 3 \cdot 1=m_{v}$ (if $v=0$ then $J_{1}$ is empty and there is $m_{0}=1$ ways again of choosing $\left.J_{1}\right)$. Finally with $J_{2} \cup J_{3}$ fixed, there are $2^{r} r$ ! ways of choosing the indices $j_{l}^{\prime}+\bar{j}_{l}^{\prime}$, all of them different, when the smallest index in $J_{2} \cup J_{3}$ is bigger than 1 , and $2^{r-1} r$ ! ways if this smallest index is 1 . Summarizing, we get

$$
w_{n}(L)= \begin{cases}m_{v} 2^{r} u !, & \text { if } 1 \notin L, \\ m_{v} 2^{r-1} u !, & \text { if } 1 \in L .\end{cases}
$$

On the other hand, we have by (5.48)

$$
\left(A(g)_{i}^{n}\right)^{u}=u ! \sum_{L \in \mathcal{L}_{n}} \prod_{j \in L}\left(g_{j}^{\prime n} \alpha_{i+j-1}^{n}\right)^{2}+\mathrm{O}_{u}\left(k_{n}^{-1-u}\right) .
$$

Therefore, by (5.56) and (5.57), we deduce that

$$
m_{v} 2^{r}\left(A(g)_{i}^{n}\right)^{u}-\bar{\Phi}(u, r)^{n}=m_{v} 2^{r-1} \sum_{L \in \mathcal{L}_{n}: 1 \in L} \prod_{j \in L}\left(g_{j}^{\prime n} \alpha_{i+j-1}^{n}\right)^{2}+\mathrm{O}_{u}\left(\Delta_{n}^{u / 2+1 / 2}\right) .
$$

Since $\left|g_{j}^{\prime n}\right| \leq K / k_{n}$ and since the number of $L \in \mathcal{L}_{n}$ such that $1 \in L$ is smaller than $k_{n}^{u-1}$, the right-hand side above is smaller than $K \Delta_{n}^{u / 2+1 / 2}$, and we deduce that $\bar{\Phi}(u, r)^{n}$ is equal to the right-hand side of (5.49). In view of (5.54), this completes the proof of (5.49).

(b) Suppose that $v$ is odd and $u=r+v / 2-1 / 2$, and recall that we need to prove that $\bar{\Phi}_{u}^{n}$ equals the right-hand side of (5.52). Again, the definition of 
$D(u)^{n}$ and the property $u=r+v / 2-1 / 2$ yield that, if $J \in D(u)^{n}$, there is a number $a$ in $\{0,1\}$ and a nonnegative integer $w \leq \frac{v-1}{2} \wedge \frac{r-s-2 a}{2}$ such that $\Phi(J, n)$ is the product of $\frac{v+s+r-w-1}{2}$ terms, all for different indices for $\chi^{n}$ with $s-w+a+\frac{v-3}{2}$ terms of type $1, w$ terms of type $2, \frac{r-s-w-2 a}{2}$ terms of type 3 and $1-a$ and $a$ term, respectively, of the types (4) and (5) described below:

(4) terms of the form $\left(g_{j}^{\prime n} \chi_{i+j-1}^{n}\right)^{3}$ or $\left(g_{j}^{\prime n}\right)^{2} g_{j+1}^{\prime n}\left(\chi_{i+j}^{n}\right)^{3}$,

(5) terms of the form $-2\left(g_{j}^{\prime n}\right)^{4} g_{j+1}^{\prime n}\left(\chi_{i+j-1}^{n}\right)^{3}\left(\chi_{i+j}^{n}\right)^{2}$ or $-2\left(g_{j}^{\prime n}\right)^{3}\left(g_{j+1}^{\prime n}\right)^{2} \times$ $\left(\chi_{i+j-1}^{n}\right)^{2}\left(\chi_{i+j}^{n}\right)^{3}$, the whole product being multiplied by -1 . It follows that $\# D(u, s)^{n} \leq K k_{n}^{(v+s+r-1) / 2}$ by the same argument as in (a) whereas $\mathbb{E}\left(|\Phi(J, n)| \mid \mathcal{G}_{i}^{n}\right) \leq K / k_{n}^{v+2 r}$ still holds. Hence, instead of (5.54) we get $\left|\bar{\Phi}(u, s)^{n}\right| \leq K \Delta_{n}^{r / 2+v / 4+1 / 4+(r-s) / 2}$. In particular, $\bar{\Phi}(u, s)^{n}=\mathrm{O}\left(\Delta_{n}^{r / 2+v / 4+1 / 2}\right)$ when $s<r$, and it thus remains to prove that $\bar{\Phi}(u, r)^{n}$ is equal to the righthand side of (5.52).

If $J \in D(u, r)^{n}$ then $\Phi(J, n)$ has $u-1$ terms of type (1) and one of type (4), and there is exactly one common index among $j_{1}, \ldots, j_{v}$ and $j_{1}^{\prime}+\bar{j}_{1}^{\prime}, \ldots, j_{s}^{\prime}+$ $\bar{j}_{s}^{\prime}$. In other words, we can associate with $J$ three sets, $J_{1}, J_{2}, J_{3}$, pairwise disjoint [with the same description than when $v$ is even, except that $\# J_{1}=$ $\frac{v-1}{2}$ and $\#\left(J_{2} \cup J_{3}\right)=r-1$ ], plus an index $l$ outside $J_{1} \cup J_{2} \cup J_{3}$ and an integer $\bar{l}$ equal to 0 or 1 , such that instead of (5.55) we have

$$
\begin{aligned}
\mathbb{E}\left(\Phi(J, n) \mid \mathcal{G}_{i}^{n}\right)= & -\left(g_{l}^{\prime n}\right)^{2} g_{l+\bar{l}}^{\prime n} \beta(3)_{i+l+\bar{l}-1}^{n} \\
& \times \prod_{j \in J_{1} \cup J_{2}}\left(g_{j}^{\prime n} \alpha_{i+j-1}^{n}\right)^{2} \prod_{j \in J_{3}}\left(g_{j-1}^{\prime n} \alpha_{i+j-1}^{n}\right)^{2} .
\end{aligned}
$$

This is equal to

$$
-\beta(3)_{i}^{n}\left(\alpha_{i}^{n}\right)^{2 u-2}\left(g_{l}^{\prime n}\right)^{3} \prod_{j \in J_{1} \cup J_{2} \cup J_{3}}\left(g_{j}^{\prime n}\right)^{2},
$$

up to $\mathrm{O}_{u}\left(k_{n}^{-2 u}\left(k_{n}^{-1}+\Gamma(\alpha, m)_{i}^{n}+\Gamma(\beta(3), m)_{i}^{n}\right)\right)$ when $Q_{n} \cap\left(\{k\} \cup J_{1} \cup J_{2} \cup\right.$ $\left.J_{3}\right)=\varnothing$ and to $\mathrm{O}_{u}\left(k_{n}^{-2 u}\right)$, otherwise. Therefore, since $\# D(u, r)^{n} \leq K k_{n}^{u}$, we deduce that

$$
\bar{\Phi}(u, r)^{n}=-\beta(3)_{i}^{n}\left(\alpha_{i}^{n}\right)^{2 u-2} \sum_{l, J_{1}, J_{2}, J_{3}}\left(g_{l}^{\prime n}\right)^{3} \prod_{j \in J_{1} \cup J_{2} \cup J_{3}}\left(g_{j}^{\prime n}\right)^{2}+R_{n},
$$

where the remainder term $R_{n}$ is like the last term in (5.52), and the sum is extended over all $l, J_{1}, J_{2}, J_{3}$ such that $\{l\}, J_{1}, J_{2}, J_{3}$ are pairwise disjoint in the set $\left\{1, \ldots, k_{n}\right\}$. Then with $R_{n}^{\prime}$ as $R_{n}$ above, we have

$$
\bar{\Phi}(u, r)^{n}=-\beta(3)_{i}^{n}\left(\alpha_{i}^{n}\right)^{2 u-2}\left(\sum_{j=1}^{k_{n}}\left(g_{j}^{\prime n}\right)^{3}\right)\left(\sum_{j=1}^{k_{n}}\left(g_{j}^{\prime n}\right)^{2}\right)^{u-1}+R_{n}^{\prime} .
$$


Then by an estimate similar to (2.10) (without the absolute value), we deduce (5.52), with $\gamma_{v, r}=-\bar{g}(2)^{r+v / 2-1 / 2} \int_{0}^{2}\left(g^{\prime}(s)\right)^{3} d s$.

Lemma 5.5. Assume Hypothesis $(S N-q)$ for some $q \geq 2$, and let $p$ be an even integer. With the notation (5.38) for $\phi(g, p)$, the variables

$$
\Psi(g, p)_{i, j}^{n}=\mathbb{E}\left(\phi(g, p)_{i, j}^{n} \mid \mathcal{G}_{i}^{n}\right)-\left(\sigma_{i}^{n} \bar{W}(g)_{i+j}^{n}\right)^{p}
$$

satisfy, for all $u \leq q / p$ and $m \geq 0$ and $0 \leq j \leq m k_{n}$,

$$
\begin{aligned}
& (5.59) \quad\left|\mathbb{E}\left(\Psi(g, p)_{i, j}^{n} \mid \mathcal{F}_{i}^{n}\right)\right| \leq K \Delta_{n}^{p / 4+1 / 4}\left(\Delta_{n}^{1 / 4}+\Gamma^{\prime}(\alpha, m)_{i}^{n}+\Gamma^{\prime}(\beta(3), m)_{i}^{n}\right), \\
& (5.60) \quad \mathbb{E}\left(\left|\Psi(g, p)_{i, j}^{n}\right|^{u} \mid \mathcal{F}_{i}^{n}\right) \leq K \Delta_{n}^{u p / 4+u / 4} .
\end{aligned}
$$

Proof. In view of (5.38), and recalling that $\sigma_{i}^{n} \bar{W}(g)_{i+j}^{n}$ is $\mathcal{G}_{i}^{n}$-measurable, we see that

$$
\begin{aligned}
\mathbb{E}\left(\phi(g, p)_{i, j}^{n} \mid \mathcal{G}_{i}^{n}\right)=\sum_{r=0}^{p / 2} \sum_{w=0}^{p-2 r} & C_{p-2 r}^{w} \rho_{p, r}\left(\sigma_{i}^{n} \bar{W}(g)_{i+j}^{n}\right)^{w} \\
& \times \mathbb{E}\left(\left(\bar{\chi}(g)_{i+j}^{n}\right)^{p-2 r-w}\left(\widehat{\chi}(g)_{i+j}^{n}\right)^{r} \mid \mathcal{G}_{i}^{n}\right) .
\end{aligned}
$$

By (3.7) and a change of the order of summation, we easily get

$$
\begin{aligned}
& \sum_{r=0}^{p / 2} \sum_{v=0}^{p / 2-r} C_{p-2 r}^{2 v} \rho_{p, r} 2^{r} m_{p-2 r-2 v}\left(\sigma_{i}^{n} \bar{W}(g)_{i+j}^{n}\right)^{2 v}\left(A(g)_{i+j}^{n}\right)^{p / 2-v} \\
& \quad=\left(\sigma_{i}^{n} \bar{W}(g)_{i+j}^{n}\right)^{p}
\end{aligned}
$$

hence

$$
\begin{aligned}
& \Psi(g, p)_{i, j}^{n}=\sum_{r=0}^{p / 2} \sum_{v=0}^{p / 2-r} C_{p-2 r}^{2 v} \rho_{p, r}\left(\sigma_{i}^{n} \bar{W}(g)_{i+j}^{n}\right)^{2 v} \times\left(\mathbb{E}\left(\left(\bar{\chi}(g)_{i+j}^{n}\right)^{p-2 r-2 v}\left(\widehat{\chi}(g)_{i+j}^{n}\right)^{r} \mid \mathcal{G}_{i}^{n}\right)\right. \\
&\left.\quad-2^{r} m_{p-2 r-2 v}\left(A(g)_{i+j}^{n}\right)^{p / 2-v}\right) \\
&+\sum_{r=0}^{p / 2} \sum_{v=0}^{p / 2-r-1} C_{p-2 r}^{2 v+1} \rho_{p, r}\left(\sigma_{i}^{n} \bar{W}(g)_{i+j}^{n}\right)^{2 v+1} \\
& \times \mathbb{E}\left(\left(\bar{\chi}(g)_{i+j}^{n}\right)^{p-2 r-2 v-1}\left(\widehat{\chi}(g)_{i+j}^{n}\right)^{r} \mid \mathcal{G}_{i}^{n}\right) .
\end{aligned}
$$

Now (5.59) is a simple consequence of (5.39) and (5.49) applied to the terms in the first sum above and of (5.42) and (5.52) for those in the second sum. Finally, (5.60) follows from (5.39), (5.49) and (5.51), plus Hölder's inequality. 
5.10. Block splitting. In this subsection we start the proof of Theorem 4.1. Due to overlapping intervals the summands involved in the definition of $V(Z, g, p, r)_{t}^{n}$ are asymptotically $k_{n}$-dependent variables, and we will use the (classical) block splitting method to ensure some "conditional" independence. Namely, we split the sum over $i$ in the definition of $\bar{V}(Z, g, p)_{t}^{n}$ into big blocks of size $m k_{n}$ ( $m$ is an integer which will eventually go to $\infty$ ) which are separated by small blocks of size $k_{n}$. The big blocks become asymptotically conditionally independent, and the small blocks become negligible as $m \rightarrow \infty$. In a second step we prove a CLT for big blocks, for any fixed $m$. We then obtain the result by standard methods.

Here we fix the integer $m \geq 2$. Recalling (5.38), the $i$ th block of size $m k_{n}$ contains $\phi(Z, g, p)_{j}^{n}$ for all $j$ between $I(m, n, i)=(i-1)(m+1) k_{n}$ and $I(m, n, i)+m k_{n}-1$. In a similar way, the $i$ th block of size $k_{n}$ corresponds to indices $j$ between $\bar{I}(m, n, i)=i(m+1) k_{n}-k_{n}$ and $\bar{I}(m, n, i)+k_{n}-1$. The number of pairs of blocks which can be accommodated without using data after time $t$ is then $i_{n}(m, t)=\left[\frac{t-\left(k_{n}-1\right) \Delta_{n}}{(m+1) k_{n} \Delta_{n}}\right]$. The "real" times corresponding to the beginnings of the $i$ th big and small blocks are then $t(m, n, i)=I(m, n, i) \Delta_{n}$ and $\bar{t}(m, n, i)=\bar{I}(m, n, i) \Delta_{n}$.

At this stage, we need some more notation. The summands in $\bar{V}(Z, g, p)_{t}$ are the $\phi(Z, g, p)_{i}^{n}$, but we will indeed show that they can be replaced by $\phi(g, p)_{i, j}^{n}$ [see (5.38) for suitable choice of $\left.i\right]$. This leads us to consider the partial sums (we drop the mention of $p$, but we keep the function $g$ )

$$
\begin{aligned}
\zeta(g, m)_{i}^{n} & =\sum_{j=0}^{m k_{n}-1} \phi(Z, g, p)_{I(m, n, i)+j}^{n}, \\
\bar{\zeta}(g, m)_{i}^{n} & =\sum_{j=0}^{k_{n}-1} \phi(Z, g, p)_{\bar{I}(m, n, i)+j}^{n}, \\
\delta(g, m)_{i}^{n} & =\sum_{j=0}^{m k_{n}-1} \phi(g, p)_{I(m, n, i), j}^{n}, \\
\bar{\delta}(g, m)_{i}^{n} & =\sum_{j=0}^{k_{n}-1} \phi(g, p)_{\bar{I}(m, n, i), j}^{n}, \\
U(g, m)_{t}^{n} & =\sum_{i=1}^{i_{n}(m, t)}\left(\zeta(g, m)_{i}^{n}-\delta(g, m)_{i}^{n}\right), \\
\bar{U}(g, m)_{t}^{n} & =\sum_{i=1}^{i_{n}(m, t)}\left(\bar{\zeta}(g, m)_{i}^{n}-\bar{\delta}(g, m)_{i}^{n}\right),
\end{aligned}
$$




$$
U^{\prime}(g, m)_{t}^{n}=\sum_{i=i_{n}(m, t)(m+1) k_{n}}^{\left[t / \Delta_{n}\right]-k_{n}} \phi(Z, g, p)_{i}^{n} .
$$

Consider the discrete time filtrations $\mathcal{F}(m)_{i}^{n}=\mathcal{F}_{I(m, n, i+1)}^{n}$ and $\overline{\mathcal{F}}(m)_{i}^{n}=$ $\mathcal{F}_{\bar{I}(m, n, i+1)}^{n}$. Observe that $\delta(g, m)_{i}^{n}$ is $\mathcal{F}(m)_{i}^{n}$-measurable and $\bar{\delta}(g, m)_{i}^{n}$ is $\overline{\mathcal{F}}(m)_{i}^{n}$-measurable, and set

$$
\begin{aligned}
\gamma(g, m)_{i}^{n} & =\mathbb{E}\left(\delta(g, m)_{i}^{n} \mid \mathcal{F}(m)_{i-1}^{n}\right), \quad \bar{\gamma}(g, m)_{i}^{n}=\mathbb{E}\left(\bar{\delta}(g, m)_{i}^{n} \mid \overline{\mathcal{F}}(m)_{i-1}^{n}\right), \\
D(g, m)_{t}^{n} & =\sum_{i=1}^{i_{n}(m, t)} \gamma(g, m)_{i}^{n}, \quad N(g, m)_{t}^{n}=\sum_{i=1}^{i_{n}(m, t)}\left(\delta(g, m)_{i}^{n}-\gamma(g, m)_{i}^{n}\right), \\
\bar{D}(g, m)_{t}^{n} & =\sum_{i=1}^{i_{n}(m, t)} \bar{\gamma}(g, m)_{i}^{n}, \quad \bar{N}(g, m)_{t}^{n}=\sum_{i=1}^{i_{n}(m, t)}\left(\bar{\delta}(g, m)_{i}^{n}-\bar{\gamma}(g, m)_{i}^{n}\right) .
\end{aligned}
$$

The key point is the following obvious relation, for any $m \geq 1$ :

$$
\begin{aligned}
\bar{V}(Z, g, p)_{t}^{n}= & N(g, m)_{t}^{n}+D(g, m)_{t}^{n}+\bar{N}(g, m)_{t}^{n}+\bar{D}(g, m)_{t}^{n} \\
& +U(g, m)_{t}^{n}+\bar{U}(g, m)_{t}^{n}+U^{\prime}(g, m)_{t}^{n}
\end{aligned}
$$

the contribution of the big blocks being $N(g, m)_{t}^{n}+D(g, m)_{t}^{n}$, whereas $\bar{N}(g$, $m)_{t}^{n}+\bar{D}(g, m)_{t}^{n}$ accounts for the small blocks, and $U(g, m)_{t}^{n}$ and $\bar{U}(g, m)_{t}^{n}$ being asymptotically negligible, whereas $U^{\prime}(g, m)_{t}^{n}$ is a border term. Note that $D(g, m)_{t}^{n}$ is a sort of drift which asymptotically cancels with the centering term in (4.1). To be more specific, the leading term for the CLT is the martingale $N(g, m)_{t}^{n}$, and we will eventually prove a CLT for it and the negligibility of the rest in the sense that

$$
\begin{aligned}
\varepsilon, t>0 \Rightarrow \lim _{m \rightarrow \infty} \limsup _{n \rightarrow \infty} \mathbb{P}\left(\sup _{s \leq t} \mid \widetilde{V}(Z, g, p)_{s}^{n}\right. & \\
& \left.\quad-\Delta_{n}^{3 / 4-p / 4} N(g, m)_{s}^{n} \mid>\varepsilon\right)=0 .
\end{aligned}
$$

LEMmA 5.6. Under $(S N-p)$ we have $\Delta_{n}^{3 / 4-p / 4} U^{\prime}(g, m)_{t}^{n} \stackrel{\mathbb{P}}{\longrightarrow} 0$ as $n \rightarrow \infty$.

Proof. The variable $U^{\prime}(g, m)_{t}^{n}$ is the sum of at most $(m+1) k_{n}$ terms $\phi(Z, g, p)_{i}^{n}$, all of them satisfying (5.45). Then the expectation of the absolute value of $\Delta_{n}^{3 / 4-p / 4} U^{\prime}(g, m)_{t}^{n}$ is less than $K_{m, p} k_{n} \Delta_{n}^{3 / 4}$ which clearly goes to 0 .

Lemma 5.7. Under $(S N-2 p)$ we have, as $n \rightarrow \infty$, and for each fixed $m$,

$$
\Delta_{n}^{3 / 4-p / 4} U(g, m)^{n} \stackrel{\text { u.c.p. }}{\longrightarrow} 0, \quad \Delta_{n}^{3 / 4-p / 4} \bar{U}(g, m) \stackrel{n}{n} \stackrel{\text { u.c.p. }}{\longrightarrow} 0 .
$$


Proof. (1) The proofs of both claims are the same, and we prove, for example, the first one. With the notation $\eta_{i}^{n}=\zeta(g, m)_{i}^{n}-\delta(g, m)_{i}^{n}$ and $\eta_{i}^{\prime n}=$ $\mathbb{E}\left(\eta_{i}^{n} \mid \mathcal{F}(m)_{i-1}^{n}\right)$, we have

$$
U(g, m)_{t}^{n}=U_{t}^{n, 1}+U_{t}^{n, 2}
$$

where

$$
U_{t}^{n, 1}=\sum_{i=1}^{i_{n}(m, t)}\left(\eta_{i}^{n}-\eta_{i}^{\prime n}\right), \quad U_{t}^{n, 2}=\sum_{i=1}^{i_{n}(m, t)} \eta_{i}^{\prime n} .
$$

Then we need to prove

$$
\Delta_{n}^{3 / 4-p / 4} U^{n, k} \stackrel{\text { u.c.p. }}{\longrightarrow} 0, \quad k=1,2 .
$$

(2) By the inequalities of Doob and Cauchy-Schwarz,

$$
\begin{aligned}
\mathbb{E}\left(\sup _{s \leq t}\left|U_{s}^{n, 1}\right|^{2}\right) & \leq 4 \sum_{i=1}^{i_{n}(m, t)} \mathbb{E}\left(\left|\eta_{i}^{n}\right|^{2}\right) \\
& \leq 4 m k_{n} \sum_{i=1}^{i_{n}(m, t)} \sum_{j=0}^{m k_{n}-1} \mathbb{E}\left(\left|\phi(Z, g, p)_{i+j}^{n}-\phi(g, p)_{i, j}^{n}\right|^{2}\right) .
\end{aligned}
$$

By (5.45) and Lemma 5.3, the right-hand side above, multiplied by $\Delta_{n}^{3 / 2-p / 2}$, goes to 0 , so (5.63) for $k=1$ follows.

For (5.63) with $k=2$, and by virtue of (5.38), and dropping $g$ from the notation, we see that it is enough to show that, for all integers $l$ between 0 and $p / 2$, we can find an AN array $\left(\delta_{i}^{n}\right)$ (depending on $l$ ) such that

$$
\begin{gathered}
0 \leq j \leq m k_{n} \Rightarrow\left|\mathbb{E}\left(\left(\bar{Z}_{i+j}^{n}\right)^{p-2 l}\left(\widehat{Z}_{i+j}^{n}\right)^{l}-\left(\bar{\kappa}_{i, j}^{n}\right)^{p-2 l}\left(\widehat{\chi}_{i+j}^{n}\right)^{l} \mid \mathcal{F}_{i}^{n}\right)\right| \\
\leq K \Delta_{n}^{p / 4+1 / 4} \delta_{i+j}^{n} .
\end{gathered}
$$

When $l=p / 2$ the second estimate (5.44) with $u=1$ gives the result, but otherwise the first estimate (5.44) with $u=1$ is not quite enough. Below we fix $l$ between 0 and $p / 2-1$, and the result will be true if we have the following:

$$
\left|\mathbb{E}\left(F_{i, j}^{n} \mid \mathcal{F}_{i}^{n}\right)\right| \leq K \Delta_{n}^{p / 4+1 / 4} \delta_{i+j}^{n},
$$

where

$$
F_{i, j}^{n}= \begin{cases}\left(\bar{\kappa}_{i, j}^{n}\right)^{p-2 l}\left(\left(\widehat{Z}_{i+j}^{n}\right)^{l}-\left(\widehat{\chi}_{i+j}^{n}\right)^{l}\right), & (\text { called Case A) } \\ \left(\widehat{\chi}_{i+j}^{n}\right)^{l}\left(\left(\bar{Z}_{i+j}^{n}\right)^{p-2 l}-\left(\bar{\kappa}_{i, j}^{n}\right)^{p-2 l}\right), & (\text { called Case B }), \\ \left(\left(\bar{Z}_{i+j}^{n}\right)^{p-2 l}-\left(\bar{\kappa}_{i, j}^{n}\right)^{p-2 l}\right)\left(\left(\widehat{Z}_{i+j}^{n}\right)^{l}-\left(\widehat{\chi}_{i, j}^{n}\right)^{l}\right), & (\text { called Case C) }\end{cases}
$$

and where again $\left(\delta_{i}^{n}\right)$ is an AN array (perhaps different for each case). 
In Cases $\mathrm{A}$ and $\mathrm{C}$ we have $F_{i, j}^{n}=0$ when $l=0$, and we have $\mid \mathbb{E}\left(F_{i, j}^{n} \mid\right.$ $\left.\mathcal{F}_{i}^{n}\right) \mid \leq K \Delta_{n}^{p / 4+1 / 2}$ when $l \geq 1$ [apply (5.39) and the second part of (5.44), plus the fact that $\Gamma^{\prime}(\sigma, m+1)_{i}^{n} \leq K$, and the Cauchy-Schwarz inequality], hence (5.64) with $\delta_{i}^{n}=\Delta_{n}^{1 / 4}$.

(3) Now we consider Case B. Recall that $\bar{Z}_{i+j}^{n}=\bar{\kappa}_{i, j}^{n}+\bar{\lambda}_{i, j}^{n}$; hence

$$
F_{i, j}^{n}=\sum_{u=1}^{p-2 l} C_{p-2 l}^{u} G_{i, j}^{u, n}, \quad G_{i, j}^{u, n}=\left(\widehat{\chi}_{i+j}^{n}\right)^{l}\left(\bar{\kappa}_{i, j}^{n}\right)^{p-2 l-u}\left(\bar{\lambda}_{i, j}^{n}\right)^{u}
$$

and we will prove (5.64) separately for each $G_{i, j}^{u, n}$. For this, we begin with a decomposition of $\bar{\lambda}_{i, j}^{n}$. Recall (5.2) and the boundedness of the coefficients. By (5.40) we have $\bar{\lambda}_{i, j}^{n}=\xi_{i, j}^{n}+\xi_{i, j}^{\prime n}$ where, with the simplifying notation $S=$ $i \Delta_{n}$ and $T=(i+j) \Delta_{n}$,

$$
\begin{aligned}
& \xi_{i, j}^{n}=\int_{T}^{T+u_{n}} g_{n}\left(s-(i+j) \Delta_{n}\right) \\
& \times\left(\left(b_{s}-b_{i}^{n}\right) d s+\left(\int_{S}^{s}\left(\widetilde{b}_{r} d r+\left(\widetilde{\sigma}_{r}-\widetilde{\sigma}_{i}^{n}\right) d W_{r}\right)\right) d W_{s}\right), \\
& \xi_{i, j}^{\prime n}=\int_{T}^{T+u_{n}} g_{n}\left(s-(i+j) \Delta_{n}\right)\left(b_{i}^{n} d s+\widetilde{\sigma}_{i}^{n}\left(W_{s}-W_{S}\right) d W_{s}+\left(M_{s}-M_{S}\right) d W_{s}\right) .
\end{aligned}
$$

Then for $v \geq 1$ and $j \leq m k_{n}$, we have

$$
\left.\begin{array}{l}
\mathbb{E}\left(\left|\xi_{i, j}^{n}\right|^{v} \mid \mathcal{F}_{i}^{n}\right) \leq K_{m, v} \Delta_{n}^{v / 2}\left(\Delta_{n}^{v / 2}+\Gamma^{\prime}(b, m+1)_{i}^{n}+\Gamma^{\prime}(\widetilde{\sigma}, m+1)_{i}^{n}\right), \\
\mathbb{E}\left(\left|\xi_{i, j}^{\prime n}\right|^{v} \mid \mathcal{F}_{i}^{n}\right) \leq K_{m, v} \Delta_{n}^{v / 4+((1 / 2) \wedge(v / 4))}, \\
\mathbb{E}\left(\left|\bar{\lambda}_{i, j}^{n}\right|^{v} \mid \mathcal{F}_{i}^{n}\right) \leq K_{m, v} \Delta_{n}^{v / 4+((1 / 2) \wedge(v / 4))} .
\end{array}\right\}
$$

(4) Next we prove that, for $u$, an odd integer,

$$
\mathbb{E}\left(\left(\bar{W}_{i+j}^{n}\right)^{u} \xi_{i, j}^{\prime n} \mid \mathcal{F}_{i}^{n}\right)=0 .
$$

We prove this separately for each of the three terms constituting $\xi_{i, j}^{\prime n}$. Since $x \mapsto x^{u}$ is an odd function, this is obvious for the term involving $b_{i}^{n}$ and also for the term involving $\widetilde{\sigma}_{i}^{n}$. For the term involving $M$, we have $\left(\bar{W}_{i+j}^{n}\right)^{u}=$ $Y+\int_{S}^{T+u_{n}} \rho_{s} d W_{s}$ for some $\mathcal{F}_{S}$-measurable variable $Y$ and process $\rho$ adapted to the filtration $\left(\mathcal{F}_{t}^{W}\right)$ generated by the Brownian motion. Since this term is a martingale increment we are left to prove $\mathbb{E}\left(U_{T+u_{n}} \mid \mathcal{F}_{S}\right)=0$ where

$$
U_{t}=\left(\int_{S}^{t} \rho_{s} d W_{s}\right)\left(\int_{T}^{T+u_{n}} g_{n}\left(s-(i+j) \Delta_{n}\right)\left(M_{s}-M_{S}\right) d W_{s}\right) .
$$

Itô's formula yields $U_{t}=M_{t}^{\prime}+\int_{T}^{t} g_{n}\left(s-(i+j) \Delta_{n}\right) \rho_{s}\left(M_{s}-M_{S}\right) d s$ for $t \geq T$ where $M^{\prime}$ is a martingale with $M_{S}^{\prime}=0$, so it is enough to prove that

$$
\mathbb{E}\left(\rho_{t}\left(M_{t}-M_{S}\right) \mid \mathcal{F}_{S}\right)=0 .
$$


But for any fixed $t \geq T$ we again have $\rho_{t}=Y_{t}^{\prime}+\int_{S}^{t} \rho_{s}^{\prime} d W_{s}$ where $Y_{t}^{\prime}$ is $\mathcal{F}_{S^{-}}$ measurable. Hence (5.67) follows from the orthogonality of $W$ and $M$, and we have (5.66).

(5) Now we use (5.39), (5.43) and (5.65), and the form of $G_{i, j}^{u, n}$ as a product of three terms at the respective powers $l, v=p-2 l-u$ and $u$. Then Hölder's inequality with the respective exponents $l^{\prime}=2 p / l$ and $v^{\prime}=4 p /(p-2 l-u)$ [so $2 l l^{\prime}=v v^{\prime}=4 p$ and (5.39) and (5.43) apply] and $u^{\prime}=4 p /(3 p+u)$ yields $\mathbb{E}\left(\left|G_{i, j}^{u, n}\right| \mid \mathcal{F}_{i}^{n}\right) \leq K \Delta_{n}^{p / 4+\left((u / 4) \wedge\left(1 / 2 u^{\prime}\right)\right)}$. Observing that $(u / 4) \wedge\left(1 / 2 u^{\prime}\right)>1 / 4$ when $u \geq 2$, we deduce that (5.64) holds for $G_{i, j}^{u, n}$ when $u \geq 2$. It remains to study $G_{i, j}^{1, n}$, which is the sum $G_{i, j}^{n}+G_{i, j}^{\prime n}$, where

$$
G_{i, j}^{n}=\left(\widehat{\chi}_{i+j}^{n}\right)^{l}\left(\bar{\kappa}_{i, j}^{n}\right)^{p-2 l-1} \xi_{i, j}^{n}, \quad G_{i, j}^{\prime n}=\left(\widehat{\chi}_{i+j}^{n}\right)^{l}\left(\bar{\kappa}_{i, j}^{n}\right)^{p-2 l-1} \xi_{i, j}^{\prime n} .
$$

By (5.39), (5.43) and (5.65), and by Hölder's inequality as above, we get

$$
\mathbb{E}\left(\left|G_{i, j}^{n}\right| \mid \mathcal{F}_{i}^{n}\right) \leq K \Delta_{n}^{p / 4+1 / 4}\left(\sqrt{\Delta_{n}}+\sqrt{\Gamma^{\prime}(b, m+1)_{i}^{n}+\Gamma^{\prime}(\widetilde{\sigma}, m+1)_{i}^{n}}\right) .
$$

Then by Lemma 5.3 we deduce that $G_{i, j}^{n}$ satisfies (5.64).

(6) It remains to study $G_{i, j}^{\prime n}$, which is also $G_{i, j}^{\prime n}=\sum_{w=0}^{p-2 l-1} C_{p-2 l-1}^{w} a(n, w, i, j)$, where $a(n, w, i, j)=\left(\sigma_{i}^{n} \bar{W}_{i+j}^{n}\right)^{p-2 l-1-w} \xi_{i, j}^{\prime n}\left(\widehat{\chi}_{i+j}^{n}\right)^{l}\left(\bar{\chi}_{i+j}^{n}\right)^{w}$. By successive conditioning, (5.51) and (5.65) yield $\left.\mathbb{E}\left(|a(n, w, i, j)| \mid \mathcal{F}_{i}^{n}\right)\right) \leq K \Delta_{n}^{p / 4+1 / 2}$ when $w$ is odd. When $w$ is even, the same argument with (5.49), plus (5.66) and the fact that $p-2 l-1-w$ is then odd yield

$$
\left|\mathbb{E}\left(a(n, w, i, j) \mid \mathcal{F}_{i}^{n}\right)\right|=\mathrm{O}_{u}\left(\Delta_{n}^{p / 4+1 / 4}\left(\Delta_{n}^{1 / 2}+\Gamma(\alpha, m)_{i}^{n}\right)\right)
$$

and by Lemma 5.3 , the proof is complete.

LEMma 5.8. Under $(S N-p)$ we have, as $n \rightarrow \infty$,

$$
\left.\begin{array}{l}
\frac{1}{\Delta_{n}^{1 / 4}}\left(\Delta_{n}^{1-p / 4} D(g, m)_{t}^{n}-\frac{m}{m+1} m_{p}(\theta \bar{g}(2))^{p / 2} \int_{0}^{t}\left|\sigma_{s}\right|^{p} d s\right) \stackrel{\text { u.c.p. }}{\longrightarrow} 0, \\
\frac{1}{\Delta_{n}^{1 / 4}}\left(\Delta_{n}^{1-p / 4} \bar{D}(g, m)_{t}^{n}-\frac{1}{m+1} m_{p}(\theta \bar{g}(2))^{p / 2} \int_{0}^{t}\left|\sigma_{s}\right|^{p} d s\right) \stackrel{\text { u.c.p. }}{\longrightarrow} 0 .
\end{array}\right\}
$$

Proof. By (2.11), $\bar{W}(g)_{i+j}^{n}$ is independent of $\mathcal{F}_{i}^{n}$, and $\mathcal{N}\left(0, \bar{g}(2)_{n} \Delta_{n}\right)$. So by virtue of $(2.6)$ and $(2.10)$ we have $\mathbb{E}\left(\left(\bar{W}(g)_{i+j}^{n}\right)^{p} \mid \mathcal{F}_{i}^{n}\right)=m_{p}(\theta \bar{g}(2))^{p / 2} \Delta_{n}^{p / 4}+$ $\mathrm{O}_{u}\left(\Delta_{n}^{p / 4+1 / 2}\right)$. Therefore by (5.60), the left-hand side of the first expression in (5.68) is smaller in absolute value than

$$
\left.\frac{K}{\Delta_{n}^{1 / 4}}\left|(m+1) k_{n} \Delta_{n} \sum_{i=1}^{i_{n}(m, t)}\right| \sigma_{t(m, n, i)}\right|^{p}-\int_{0}^{t}\left|\sigma_{s}\right|^{p} d s \mid+K t(m+1) \Delta_{n}^{1 / 4}
$$




$$
+K t(m+1) \sqrt{\Delta_{n}} \sum_{i=1}^{i_{n}(m, t)}\left(\Gamma^{\prime}(\alpha, m)_{I(m, n, i)}^{n}+\Gamma^{\prime}(\beta(3), m)_{I(m, n, i)}^{n}\right) .
$$

The second term above goes to 0 , as the last term (locally uniformly in $t$, in probability) by Lemma 5.3. The first term goes to 0 locally uniformly in $t$ in probability as well because of our Hypothesis (K) (see, e.g., [13]). Therefore the first assertion in (5.68) holds, and the second one is proved in the same way.

Lemma 5.9. Under $(S N-2 p)$ we have for all $m \geq 2$ and $t>0$,

$$
\mathbb{E}\left(\sup _{s \leq t}\left(\Delta_{n}^{3 / 4-p / 4} \bar{N}(g, m)_{s}^{n}\right)^{2}\right) \leq \frac{K t}{m} .
$$

Proof. By Doob's inequality, the left-hand side above is smaller than

$$
4 \Delta_{n}^{3 / 2-p / 2} \sum_{i=1}^{i_{n}(m, t)} \mathbb{E}\left(\left(\bar{\delta}(g, m)_{i}^{n}\right)^{2}\right),
$$

whereas (5.45) yields $\mathbb{E}\left(\left(\bar{\delta}(g, m)_{i}^{n}\right)^{2}\right) \leq K \Delta_{n}^{p / 2-1}$. Since $i_{n}(m, t) \leq K t / m \sqrt{\Delta_{n}}$, we readily deduce the result.

5.11. An auxiliary CLT. From what precedes, the leading processes for the behavior of $\bar{V}(g, p)^{n}$ are the processes $N(g, m)^{n}$, and here we prove a CLT for the vector $\left(N\left(g_{i}, m\right)^{n}\right)_{1 \leq i \leq d}$ when $m \geq 2$ is fixed and $\left(g_{i}\right)_{1 \leq i \leq d}$ is a family of functions satisfying (2.7). We first complement the notation (3.13). For $\zeta, \eta \in \mathbb{R}$ and $p>0$ and $m \geq 1$ we set

$$
\left.\begin{array}{l}
\mu_{2 p}^{m}(g, h ; \eta, \zeta)=\sum_{r, r^{\prime}=0}^{p / 2} \rho_{p, r} \rho_{p, r^{\prime}}\left(2 \zeta^{2} \bar{g}^{\prime}(2)\right)^{r}\left(2 \zeta^{2} \bar{h}^{\prime}(2)\right)^{r^{\prime}}, \\
\int_{0}^{m} \int_{0}^{m} \mathbb{E}^{\prime}\left(\left(\eta L(g)_{s}+\zeta L^{\prime}(g)_{s}\right)^{p-2 r}\left(\eta L(h)_{t}+\zeta L^{\prime}(h)_{t}\right)\right)^{p-2 r^{\prime}} d s d t, \\
\bar{\mu}_{2 p}^{m}(g, h ; \eta, \zeta)=\frac{1}{m+1}\left(\mu_{2 p}^{m}(g, h ; \eta, \zeta)-m^{2} \mu_{p}(g ; \eta, \zeta) \mu_{p}(h ; \eta, \zeta)\right) .
\end{array}\right\}
$$

Exactly as in Lemma 3.5 , the matrix with entries $\bar{\mu}_{2 p}^{m}\left(g_{i}, g_{j} ; \eta, \zeta\right)$ is symmetric nonnegative.

Proposition 5.10. Assume $(S N-4 p)$, and let $m \geq 2$. The sequence of d-dimensional processes with components $\Delta_{n}^{3 / 4-p / 4} N\left(g_{i}, m\right)$ converges stably in law to a process of the following form:

$$
\left(\theta^{1 / 2-p / 2} \sum_{j=1}^{d} \int_{0}^{t} \psi_{i j}^{m}\left(\theta \sigma_{s}, \alpha_{s}\right) d B_{s}^{j}\right)_{1 \leq i \leq d},
$$


where $B$ is as in Theorem 4.1 and $\psi^{m}$ is a measurable $d \times d$ matrix-valued function such that $\left(\psi^{m} \psi^{m \star}\right)(\eta, \zeta)$ is the matrix with entries $\bar{\mu}_{2 p}^{m}\left(g_{i}, g_{j} ; \eta, \zeta\right)$, as defined by (5.69).

We begin with a lemma, for which we use the notation $\Gamma$ of Lemma 5.1.

Lemma 5.11. Let $m \geq 2$ and $s \in \Gamma$ and $i_{n}=\min \left(i: I(m, n, i) \Delta_{n} \geq s\right)$. Then under $(S N-4 p)$ we have the following almost sure convergences:

$$
\begin{aligned}
& \Delta_{n}^{1 / 2-p / 4} \gamma(g, m)_{i_{n}}^{n} \\
& \quad \rightarrow m m_{p} \theta^{1+p / 2} \bar{g}(2)^{p / 2}\left|\sigma_{s}\right|^{p}=m \theta^{1-p / 2} \mu_{p}\left(g ; \theta \sigma_{s}, \alpha_{s}\right), \\
& \Delta_{n}^{1-p / 2} \mathbb{E}\left(\delta(g, m)_{i_{n}}^{n} \delta(h, m)_{i_{n}}^{n} \mid \mathcal{F}(m)_{i_{n}-1}^{n}\right) \\
& \quad \rightarrow \theta^{2-p} \mu_{2 p}^{m}\left(g, h ; \theta \sigma_{s}, \alpha_{s}\right) .
\end{aligned}
$$

Proof. We set $i_{n}^{\prime}=I\left(m, n, i_{n}\right)$ and $s_{n}=i_{n}^{\prime} \Delta_{n}$, which converges to $s$. Both results are consequences of Lemma 5.1: first, by (5.60) with $u=1$, (5.71) follows from

$$
\Delta_{n}^{1 / 2-p / 4} \mathbb{E}\left(\sum_{j=0}^{m k_{n}-1}\left|\sigma_{s_{n}} \bar{W}(g)_{i_{n}^{\prime}+j}^{n}\right|^{p} \mid \mathcal{F}_{s_{n}}\right) \rightarrow m m_{p} \theta^{1+p / 2} \bar{g}(2)^{p / 2}\left|\sigma_{s}\right|^{p} .
$$

Then we apply Lemma 5.1 with $d=1$ and $g_{1}=g$ and with the functions

$$
f_{n}(x, y, z)=\frac{1}{k_{n}} \sum_{j=0}^{m k_{n}-1}\left|x\left(j / k_{n}\right)\right|^{p}, \quad f(x, y, z)=\int_{0}^{m}|x(s)|^{p} d s,
$$

which satisfy (5.24) and $f_{n} \rightarrow f$ pointwise. The left-hand (right) side of (5.73) is equal to $\Delta_{n}^{1 / 2-p / 4} / k_{n}^{p / 2-1}$ times $\left(\theta^{1-p / 2}\right.$ times) the left-hand (right) side of (5.25); hence (5.71) holds.

For (5.72) we apply Lemma 5.1 with $d=2$ and $g_{1}=g$ and $g_{2}=h$ and the functions

$$
\begin{aligned}
& f_{n}\left(\left(x, x^{\prime}\right),\left(y, y^{\prime}\right),\left(z, z^{\prime}\right)\right) \\
& =\sum_{r, r^{\prime}=0}^{p / 2} \rho_{p, r} \rho_{p, r^{\prime}} \frac{1}{k_{n}^{2}} \sum_{j, j^{\prime}=0}^{m k_{n}-1}\left(x\left(\frac{j}{k_{n}}\right)+y\left(\frac{j}{k_{n}}\right)\right)^{p-2 r} \\
& \quad \times\left(x^{\prime}\left(\frac{j^{\prime}}{k_{n}}\right)+y^{\prime}\left(\frac{j^{\prime}}{k_{n}}\right)\right)^{p-2 r^{\prime}} z\left(\frac{j}{k_{n}}\right)^{r} z^{\prime}\left(\frac{j^{\prime}}{k_{n}}\right)^{r^{\prime}}, \\
& f\left(\left(x, x^{\prime}\right),\left(y, y^{\prime}\right),\left(z, z^{\prime}\right)\right)
\end{aligned}
$$




$$
\begin{gathered}
=\sum_{r, r^{\prime}=0}^{p / 2} \rho_{p, r} \rho_{p, r^{\prime}} \int_{0}^{m} \int_{0}^{m}(x(s)+y(s))^{p-2 r}\left(x^{\prime}(t)+y^{\prime}(t)\right)^{p-2 r^{\prime}} \\
\times z(s)^{r} z^{\prime}(t)^{r^{\prime}} d s d t,
\end{gathered}
$$

which satisfy (5.24) and $f_{n} \rightarrow f$ pointwise. The left-hand (right) side of (5.72) is equal to $\Delta_{n}^{1-p / 2} / k_{n}^{p-2}$ times $\left(\theta^{2-p}\right.$ times) the left-hand (right) side of (5.25); hence (5.72) holds.

Proof of Proposition 5.10. (1) As is well known, and with the $d$ dimensional variables with components $\xi_{i}^{n, k}=\Delta_{n}^{1 / 2-p / 4}\left(\delta\left(g_{k}, m\right)_{i}^{n}-\gamma\left(g_{k}, m\right)_{i}^{n}\right)$ (which are martingale differences), it suffices to prove the following three convergences, for all $t>0$ and all bounded martingales $N$ :

$$
\begin{gathered}
\sqrt{\Delta_{n}} \sum_{i=1}^{i_{n}(m, t)} \mathbb{E}\left(\xi_{i}^{n, k} \xi_{i}^{n, l} \mid \mathcal{F}(m)_{i-1}^{n}\right) \stackrel{\mathbb{P}}{\longrightarrow} \theta^{1-p} \int_{0}^{t} \bar{\mu}_{2 p}^{m}\left(g_{k}, g_{l} ; \theta \sigma_{s}, \alpha_{s}\right) d s \\
\Delta_{n} \sum_{i=1}^{i_{n}(m, t)} \mathbb{E}\left(\left\|\xi_{i}^{n}\right\|^{4} \mid \mathcal{F}(m)_{i-1}^{n}\right) \stackrel{\mathbb{P}}{\longrightarrow} 0 \\
\Delta_{n}^{1 / 4} \sum_{i=1}^{i_{n}(m, t)} \mathbb{E}\left(\xi_{i}^{n}\left(N_{i(m+1) u_{n}}-N_{(i-1)(m+1) u_{n}}\right) \mid \mathcal{F}(m)_{i-1}^{n}\right) \stackrel{\mathbb{P}}{\longrightarrow} 0
\end{gathered}
$$

(we use Theorem IX.7.28 of [16], with $Z$ being a bounded martingale of the form $Z_{t}=\int_{0}^{t} u_{s} d W_{s}$ for some predictable process $u$ with values in $\left.(0,1]\right)$.

(2) Equation (5.45) and Hölder's inequality imply $\mathbb{E}\left(\left|\delta\left(g_{k}, m\right)_{i}^{n}\right|^{4} \mid \mathcal{F}(m)_{i-1}^{n}\right) \leq$ $K_{m} \Delta_{n}^{p-2}$. Then the expected value of the left-hand side of (5.75) is smaller than $K_{m} \sqrt{\Delta_{n}}$, yielding (5.75). The proof of (5.74) is similar to the proof of Theorem 3.3. Set $\zeta_{i}^{n}=\mathbb{E}\left(\xi_{i}^{n, k} \xi_{i}^{n, l} \mid \mathcal{F}(m)_{i-1}^{n}\right)$, and $\gamma_{s}=\bar{\mu}_{2 p}^{m}\left(g_{k}, g_{l} ; \theta \sigma_{s}, \alpha_{s}\right)$. Since $k_{n} \sqrt{\Delta_{n}} \rightarrow \theta$, we need to show that

$$
(m+1) k_{n} \Delta_{n} \sum_{i=1}^{i_{n}(m, t)} \zeta_{i}^{n} \stackrel{\mathbb{P}}{\longrightarrow}(m+1) \theta^{2-p} \int_{0}^{t} \gamma_{s} d s .
$$

Note that $\left|\zeta_{i}^{n}\right| \leq K_{m}$. Then, as for Theorem 3.3, the above will follow from the fact that for any $s \in \Gamma$, and with the notation $i_{n}$ of Lemma 5.11, we have [similar to (5.47)]

$$
\zeta_{i_{n}}^{n} \rightarrow \theta^{2-p} \gamma_{s} \quad \text { a.s. }
$$

Then (5.77) readily follows from Lemma 5.11 and (5.69), once observed that

$$
\zeta_{i_{n}}^{n}=\Delta_{n}^{1-p / 2}\left(\mathbb{E}\left(\delta\left(g_{k}, m\right)_{i_{n}}^{n} \delta\left(g_{l}, m\right)_{i_{n}}^{n} \mid \mathcal{F}(m)_{i_{n}-1}^{n}\right)-\gamma\left(g_{k}, m\right)_{i_{n}}^{n} \gamma\left(g_{l}, m\right)_{i_{n}}^{n}\right) .
$$


(3) Now we turn to (5.76), which we prove for the first component, say with $g=g_{1}$. For simplicity we write $D_{i}^{n}(Y)=Y_{i(m+2) u_{n}}-Y_{(i-1)(m+2) u_{n}}$ for any process $Y$. In view of the definition of $\xi_{i}^{n}$, and since $N$ is a martingale, it is enough to prove that

$$
\Delta_{n}^{3 / 4-p / 4} \sum_{i=1}^{i_{n}(m, t)} \mathbb{E}\left(\delta(g, m)_{i}^{n} D_{i}^{n}(N) \mid \mathcal{F}(m)_{i-1}^{n}\right) \stackrel{\mathbb{P}}{\longrightarrow} 0 .
$$

Observe that (5.58) and (5.60) yield $\delta(g, m)_{i}^{n}=\delta_{i}^{\prime n}+\Psi_{i}^{\prime n}$ where

$\delta_{i}^{\prime n}=\sum_{j=0}^{m k_{n}-1}\left(\sigma_{I(m, n, i)}^{n} \bar{W}(g)_{I(m, n, i)+j}^{n}\right)^{p}, \quad \mathbb{E}\left(\left|\Psi_{i}^{\prime n}\right|^{2} \mid \mathcal{F}(m)_{i-1}^{n}\right) \leq K \Delta_{n}^{p / 2-1 / 2}$.

Since $N$ is bounded, $\sum_{i=1}^{i_{n}(m, t)} \mathbb{E}\left(\left(D_{i}^{n}(N)\right)^{2}\right) \leq K$ and the Cauchy-Schwarz inequality yields

$$
\begin{aligned}
\mathbb{E}\left(\Delta_{n}^{3 / 4-p / 4} \sum_{i=1}^{i_{n}(m, t)}\left|\Psi_{i}^{\prime n}\right|\left|D_{i}^{n}(N)\right|\right) & \leq K \Delta_{n}^{3 / 4-p / 4}\left(\mathbb{E}\left(\sum_{i=1}^{i_{n}(m, t)}\left(\Psi_{i}^{\prime n}\right)^{2}\right)\right)^{1 / 2} \\
& \leq K \Delta_{n}^{1 / 4}
\end{aligned}
$$

Therefore it remains to prove that

$$
\Delta_{n}^{3 / 4-p / 4} \sum_{i=1}^{i_{n}(m, t)} \mathbb{E}\left(\delta_{i}^{\prime n} D_{i}^{n}(N) \mid \mathcal{F}(m)_{i-1}^{n}\right) \stackrel{\mathbb{P}}{\longrightarrow} 0 .
$$

(4) Observe that, by $\mathbb{E}\left(\left|\delta_{i}^{\prime n}\right|^{2}\right) \leq K \Delta_{n}^{p / 2-1}$ and the Cauchy-Schwarz inequality,

$$
\Delta_{n}^{3 / 4-p / 4} \sum_{i=1}^{i_{n}(m, t)} \mathbb{E}\left(\left|\delta_{i}^{\prime n} D_{i}^{n}(N)\right|\right) \leq K \sqrt{\mathbb{E}\left(N_{t}^{2}\right)} .
$$

Then the set of all square-integrable martingales $N$ satisfying (5.78) is closed under $\mathbb{L}^{2}$-convergence, and thus for proving (5.78) we can use the following scheme:

(a) Prove (5.78) when $N$ is $\left(\mathcal{F}_{t}^{(0)}\right)$-adapted and orthogonal to $W$.

(b) Prove (5.78) when $N_{t}=\int_{0}^{t} \gamma_{s} d W_{s}$ where $\gamma$ is $\left(\mathcal{F}_{t}^{(0)}\right)$-adapted and constant in time over intervals $\left(t_{i-1}, t_{i}\right]$ with $t_{0}=0$ and $t_{q}=\infty$ for some $q$.

(c) Conclude from the closeness proved before that (5.78) holds for all $N \in \mathcal{N}^{0}$, the set of all bounded $\left(\mathcal{F}_{t}^{(0)}\right)$-martingales.

(d) Prove (5.78) when $N$ is in the set $\mathcal{N}^{1}$ of all martingales having $N_{\infty}=$ $f\left(\chi_{t_{1}}, \ldots, \chi_{t_{q}}\right)$ where $f$ is any Borel bounded on $\mathbb{R}^{q}$ and $t_{1}<\cdots<t_{q}$ and $q \geq 1$. 
(e) Since $\mathcal{N}^{0} \cup \mathcal{N}^{1}$ is a total subset of the set of all square-integrable $\left(\mathcal{F}_{t}\right)$ martingales, conclude once more from the closeness that (5.78) holds for all such $N$.

We are thus left to prove (a), (b) and (d), and for these we can reproduce Step 5 of the proof of Lemma 5.7 in [14].

5.12. Proof of Theorem 4.1. By localization we may assume (SN-4p) and Hypothesis (SK). Then, upon applying Lemmas 5.6, 5.7, 5.8 and 5.9, we readily deduce (5.62) from (4.1) and (5.61).

On the other hand, we fix the $d$-dimensional Brownian motion $B$ in (5.70) and (4.2) (the same in both). Proposition 5.10 yields, for each fixed $m$, that $\Delta_{n}^{3 / 4-p / 4} N(g, m)$ stably converges in law to the right-hand side of $(5.70)$. Next, the following property is implicitly proved in the proof of Lemma 3.5 in Section 5.3 (with $T$ playing the role of $m$ here):

$$
\bar{\mu}_{2 p}^{m}(g, h ; \eta, \zeta) \rightarrow \bar{\mu}_{2 p}(g, h ; \eta, \zeta) \quad \text { as } m \rightarrow \infty .
$$

Then we see that we can choose suitable versions for the square-roots $\psi$ and $\psi^{m}$ in such a way that $\psi^{m}(\eta, \zeta) \rightarrow \psi(\eta, \zeta)$ for all $\eta, \zeta$. Then (5.70) converges in probability toward (4.2). The result then follows from (5.62) in a standard way.

5.13. Theorem 4.4: A key decomposition. Here we start the proof of Theorem 4.4 , by providing a decomposition for the processes $\widetilde{V}^{*}(g, p)^{n}$ of $(4.3)$. So we fix $p>3$, and assume $\alpha$ càdlàg. By localization we can and will assume $(\mathrm{SN}-2 p)$ and Hypothesis (SK) without special mention.

The choice of the exhausting sequence $\left(T_{m}\right)$ in (4.6) is arbitrary, but a convenient choice is as follows: for $q \geq 1$ we consider the successive jump times $(T(q, m): m \geq 1)$ of the Poisson process $\mu((0, t] \times\{z: 1 / q<\gamma(z) \leq 1 /(q-1)\})$ where $\gamma$ is the function occurring in Hypothesis (SH). Those stopping times have pairwise disjoint graphs as $m$ and $q$ vary, and $\left(T_{m}\right)_{m \geq 1}$ denotes any reordering of the double sequence $(T(q, m): q, m \geq 1)$. We complete this sequence by setting $T_{0}=0$.

Let $P_{q}$ be the set of all $m \geq 1$ such that $T_{m}=T\left(q^{\prime}, m^{\prime}\right)$ for some $m^{\prime} \geq 1$ and some $q^{\prime} \leq q$. We consider the following processes [compare with (5.5)]:

$$
\left.\begin{array}{l}
X^{q}=\left(\delta 1_{\{z: \gamma(z)>1 / q\}}\right) * \underline{\mu}, \quad M^{q}=\left(\delta 1_{\{z: \gamma(z) \leq 1 / q\}}\right) *(\underline{\mu}-\underline{\nu}), \\
X^{\prime q}=X-X^{q}, \quad X^{\prime \prime q}=X^{\prime q}-M^{q}, \\
Z^{\prime q}=X^{\prime q}+\chi, \quad Z^{\prime \prime q}=X^{\prime \prime q}+\chi .
\end{array}\right\}
$$

So $X^{\prime \prime q}$ satisfies (2.14) with the same $\sigma$ as in (2.13) and a bounded drift given by

$$
b_{t}^{q}=b_{t}-\int_{\{z: \gamma(z)>1 / q,|\delta(t, z)| \leq 1\}} \delta(t, z) \lambda(d z) .
$$


Here, $X^{q}$ is the sum of "big" jumps, and this is the part of $X$ which essentially imports for our CLT: more precisely, we single out the summands in $V(X, g, p, 0)_{t}^{n}$ which involve at least one jump of $X^{q}$ [after centering this is the process $Y(q, g)$ defined below in (5.82)]. We obtain a CLT for these processes $Y(q, g)$ in a relatively simple way, and then prove that the contribution of the other summands is negligible, when $n$ and $q$ are large (so the cut-off level $1 / q$ for the "big" jumps is small).

We denote by $\Omega_{n}(t, q)$ the set of all $\omega$ such that for any $m, m^{\prime} \in P_{q}$ with $T_{m}(\omega) \leq t$, we have $2 u_{n}<T_{m}(\omega) \leq t-4 u_{n}$, and $\left|T_{m}(\omega)-T_{m^{\prime}}(\omega)\right|>4 u_{n}$, and also $T_{m}(\omega) / \Delta_{n}$ is not an integer. Since the set $\left\{T_{m}: m \in P_{q}\right\}$ is locally finite and $\mathbb{P}\left(T_{m}=t\right)=0$ for all $m$ and $t \geq 0$, we have

$$
\Omega_{n}(t, q) \rightarrow \Omega \quad \text { a.s., as } n \rightarrow \infty .
$$

Next, we denote by $\widetilde{V}^{*}(X, g, p)^{n}$ the process defined by (4.3) to emphasize the dependency on $X$, and likewise we have $\widetilde{V}^{*}\left(X^{\prime q}, g, p\right)^{n}$. Then a (relatively) simple computation shows the following key property which holds on the set $\Omega_{n}(t, q)$ :

$$
\begin{aligned}
\widetilde{V}^{*}(X, g, p)_{t}^{n} & =\widetilde{V}^{*}\left(X^{\prime q}, g, p\right)_{t}^{n}+Y(q, g)_{t}^{n}, Y(q, g)_{t}^{n} \\
& =\sum_{m \in P_{q}: T_{m} \leq t} \zeta(q, g)_{m}^{n},
\end{aligned}
$$

where, with the random integer $I_{m}^{n}=\left[T_{m} / \Delta_{n}\right]$, we have set

$$
\begin{aligned}
\zeta(q, g)_{m}^{n}=\frac{1}{\Delta_{n}^{1 / 4} k_{n}}\left(\sum _ { j = 1 } ^ { k _ { n } - 1 } \left(\left|\overline{Z^{\prime q}}(g)_{I_{m}^{n}+1-j}^{n}+g_{j}^{n} \Delta X_{T_{m}}\right|^{p}\right.\right. \\
\left.-\left|\overline{Z^{\prime q}}(g)_{I_{m}^{n}+1-j}^{n}\right|^{p}-\left|g_{j}^{n} \Delta X_{T_{m}}\right|^{p}\right) \\
\left.+\left(\bar{g}(p)_{n}-k_{n} \bar{g}(p)\right)\left|\Delta X_{T_{m}}\right|^{p}\right) .
\end{aligned}
$$

[Note that $\overline{Z^{\prime q}}(g)_{I_{m}^{n}-j}^{n}$ possibly involves $\Delta_{l}^{n} Z^{\prime}(q)$ for negative integers $l$, although this does not occur on the set $\Omega_{n}(t, q)$ when $m \in P_{q}$ and $j \leq 2 k_{n}$; however, to have such variables defined everywhere, we make the convention $\Delta_{i}^{n} Y=0$ for any process $Y$ when $i \leq 0$.]

5.14. The processes $Y(q, g)^{n}$. The aim of this subsection is to prove the following proposition.

Proposition 5.12. If $q \geq 1$ and $t \geq 0$ are fixed, and in the same setting as before, we have (with $\stackrel{\mathcal{L}-(s)}{\longrightarrow}$ denoting the stable convergence in law)

$$
\left(Y\left(q, g_{l}\right)_{t}^{n}\right)_{1 \leq l \leq d} \stackrel{\mathcal{L}-(s)}{\longrightarrow} U(p, q)_{t},
$$


where $U(p, q)$ is the d-dimensional process associated with the functions $\left(g_{l}\right)$ by (4.6), except that the sums are taken over $m \in P_{q}$ only.

We start with the following lemma which describes the behavior of the variables (with $m \in P_{q}$ ):

$$
\eta(q, g)_{m}^{n}=\frac{1}{\Delta_{n}^{1 / 4} k_{n}} \sum_{j=1}^{k_{n}-1}\left\{g_{j}^{n}\right\}^{p-1} \overline{Z^{\prime q}}(g)_{I_{m}^{n}-j+1}^{n} .
$$

The two key properties for the next lemma are the approximation (5.1) and the fact that the times $T_{m}$ are independent of $W$ and with an absolutely continuous law. Recall that we have the family $\left(g_{l}\right)_{1 \leq l \leq d}$ of $d$ weight functions with $\left(U_{m-}, U_{m+}, \bar{U}_{m-}, \bar{U}_{m+}\right)$ associated as before Theorem 4.4 .

LEMmA 5.13. For any $q \geq 1$, the $\left(\mathbb{R}^{d}\right)^{\mathbb{N}^{\star}}$-valued variables $(\eta(q$, $\left.\left.g_{l}\right)_{m}^{n}\right)_{1 \leq l \leq d, m \in P_{q}}$ converge stably in law, as $n \rightarrow \infty$, to $\left(\eta_{m}\right)_{m \in P_{q}}$, where $\eta_{m}$ is the d-dimensional variable given by

$$
\eta_{m}=\sqrt{\theta} \sigma_{T_{m}-} U_{m-}+\sqrt{\theta} \sigma_{T_{m}} U_{m+}+\frac{\alpha_{T_{m}-}}{\sqrt{\theta}} \bar{U}_{m-}+\frac{\alpha_{T_{m}}}{\sqrt{\theta}} \bar{U}_{m+}
$$

Proof. As is well known, it is enough to prove the result for any finite subset of $m$ 's, say in a finite subset $P_{q}^{\prime}$ of $P_{q}$. Since $q$ is fixed, we drop it from the notation, writing $Z^{\prime}=Z^{\prime q}, Z^{\prime \prime}=Z^{\prime \prime q}, M=M^{q}, X^{\prime}=X^{\prime q}$ and $X^{\prime \prime}=X^{\prime \prime q}$.

(1) The times $\left(T_{m}: m \in P_{q}\right)$ are independent of $W$, and also of the restriction $\underline{\mu}(q)$ of the Poisson measure $\underline{\mu}$ to the set $\mathbb{R}_{+} \times\{z: \gamma(z) \leq 1 / q\}$. Hence if $\mathcal{H}_{t}=\mathcal{F}_{t} \vee \sigma\left(T_{m}: m \in P_{q}\right)$, the process $W$ is a Brownian motion and the measure $\mu(q)$ a Poisson measure with compensator $\underline{\nu}(q)$, the restriction of $\underline{\nu}$ to $\mathbb{R}_{+} \overline{\times}\{z: \gamma(z) \leq 1 / q\}$ again, relative to the filtration $\left(\mathcal{H}_{t}\right)$. Thus $X^{\prime \prime}$ admits the same representation (2.14) and $M$ has the same form (5.79) relative to the two filtrations $\left(\mathcal{F}_{t}\right)$ and $\left(\mathcal{H}_{t}\right)$. With the random integers $I_{m}^{n}$ being $\mathcal{H}_{0}$-measurable, we deduce from (5.3) and (5.39) and (5.4) for $M$ together with $\left|\left(g_{l}\right)_{n}(s)\right| \leq K$ that, for $v \in(0,2 p]$ and $j \in \mathbb{Z}$ and $i=l, \ldots, d$,

$$
\left.\begin{array}{l}
\mathbb{E}\left(\left|\Delta_{I_{n}^{m}+j}^{n} X^{\prime \prime}\right|^{v}\right) \leq K_{v, q} \Delta_{n}^{v / 2}, \\
\mathbb{E}\left(\left|\bar{M}\left(g_{l}\right)_{I_{m}^{n}+j}^{n}\right|^{2}\right) \leq K \sqrt{\Delta_{n}}, \\
\mathbb{E}\left(\left|\bar{X}^{\prime \prime}\left(g_{l}\right)_{I_{m}^{n}+j}^{n}\right|^{v}+\left|\bar{Z}^{\prime \prime}\left(g_{l}\right)_{I_{m}^{n}+j}^{n}\right|^{v}\right) \leq K_{v, q} \Delta_{n}^{v / 4} .
\end{array}\right\}
$$

Now if $f$ is a bounded function on $\mathbb{R}$, arguments similar to the one giving (5.41) [relative to the filtration $\left(\mathcal{H}_{t}\right)$ and using that $\sigma$ is càdlàg and bounded 
and the drift $b^{q}$ is also bounded], we obtain that if $2 \leq k_{n}^{\prime} \leq 2 k_{n}$,

$$
\left.\begin{array}{l}
\mathbb{E}\left(\left|\sum_{j=0}^{k_{n}^{\prime}} f\left(j / k_{n}\right) \Delta_{I_{n}^{m}-j}^{n} X^{\prime \prime}-\sigma_{T_{m}-} \sum_{j=0}^{k_{n}^{\prime}} f\left(j / k_{n}\right) \Delta_{I_{n}^{m}-j}^{n} W\right|^{v}\right) \\
\quad=\mathrm{o}_{u}\left(\Delta_{n}^{v / 4}\right), \\
\mathbb{E}\left(\left|\sum_{j=2}^{k_{n}^{\prime}} f\left(j / k_{n}\right) \Delta_{I_{n}^{m}+j}^{n} X^{\prime \prime}-\sigma_{T_{m}} \sum_{j=2}^{k_{n}^{\prime}} f\left(j / k_{n}\right) \Delta_{I_{n}^{m}+j}^{n} W\right|^{v}\right) \\
\quad=\mathrm{o}_{u}\left(\Delta_{n}^{v / 4}\right) .
\end{array}\right\}
$$

Moreover $A_{n}=\sum_{j=0}^{k_{n}^{\prime}} f\left(j / k_{n}\right) \Delta_{I_{n}^{m}-j}^{n} M$, say, can be written as $\delta^{n} \star(\underline{\mu}(q)-$ $\underline{\nu}(q))_{T_{m}}-\delta^{n} \star(\mu(q)-\underline{\nu}(q))_{T_{m}-2 u_{n}}$ for some predictable function $\delta^{n}$ satisfying $\left|\delta^{n}(t, z)\right| \leq K \gamma(z)$. Then a well-known result (see, e.g., Lemma 5.12 of [13], used with $2 u_{n}$ instead of $\Delta_{n}$ and $\eta=\sqrt{u_{n}}$, and relative to the filtration $\left(\mathcal{H}_{t}\right)$ ) says that $A_{n} / \sqrt{u_{n}} \stackrel{\mathbb{P}}{\longrightarrow} 0$. The same holds if we take the indices $I_{l}^{n}+j$ instead of $I_{m}^{n}-j$, and thus

$$
\begin{aligned}
& \frac{1}{\Delta_{n}^{1 / 4}} \sum_{j=0}^{k_{n}^{\prime}} f\left(j / k_{n}\right) \Delta_{I_{n}^{m}-j}^{n} M \stackrel{\mathbb{P}}{\longrightarrow} 0, \\
& \frac{1}{\Delta_{n}^{1 / 4}} \sum_{j=2}^{k_{n}^{\prime}} f\left(j / k_{n}\right) \Delta_{I_{n}^{m}+j}^{n} M \stackrel{\mathbb{P}}{\longrightarrow} 0 .
\end{aligned}
$$

(2) We put for $i \geq 0$ and any weight function $g$,

$$
\begin{aligned}
& G(g)_{i-}^{n}=\frac{1}{k_{n}} \sum_{j=i+2}^{k_{n}-1}\left\{g_{j}^{n}\right\}^{p-1} g_{j-i-1}^{n}, \quad G(g)_{0}^{n}=\frac{1}{k_{n}} \sum_{j=1}^{k_{n}-1}\left\{g_{j}^{n}\right\}^{p-1} g_{j}^{n}, \\
& G(g)_{i+}^{n}=\frac{1}{k_{n}} \sum_{j=1}^{k_{n}-i}\left\{g_{j}^{n}\right\}^{p-1} g_{j+i-1}^{n}, \\
& \bar{G}(g)_{i-}^{n}=\sum_{j=i+1}^{k_{n}-1}\left\{g_{j}^{n}\right\}^{p-1} g_{j-i}^{\prime n}, \quad \bar{G}(g)_{i+}^{n}=\sum_{j=1}^{k_{n}-i}\left\{g_{j}^{n}\right\}^{p-1} g_{j+i}^{\prime n} .
\end{aligned}
$$

Then a (tedious) computation shows that

$$
\begin{aligned}
\eta(q, g)_{m}^{n}=\frac{1}{\Delta_{n}^{1 / 4}} & \left(\sum_{i=0}^{k_{n}-3} G(g)_{i-}^{n} \Delta_{I_{m}^{n}-i}^{n} X^{\prime}\right. \\
& \left.+G(g)_{0}^{n} \Delta_{I_{m}^{n}+1}^{n} X^{\prime}+\sum_{i=2}^{k_{n}-1} G(g)_{i+}^{n} \Delta_{I_{m}^{n}+i}^{n} X^{\prime}\right)
\end{aligned}
$$




$$
-\frac{1}{\Delta_{n}^{1 / 4} k_{n}}\left(\sum_{i=0}^{k_{n}-2} \bar{G}(g)_{i-}^{n} \chi_{I_{m}^{n}-i}^{n}+\sum_{i=1}^{k_{n}-1} \bar{G}(g)_{i+}^{n} \chi_{I_{m}^{n}+i}^{n}\right) .
$$

Moreover, if

$$
\begin{aligned}
& H_{-}(g, t)=\int_{t}^{1}\{g(s)\}^{p-1} g(s-t) d s, \\
& H_{+}(g, t)=\int_{0}^{1-t}\{g(s)\}^{p-1} g(s+t) d s, \\
& \bar{H}_{-}(g, t)=\int_{t}^{1}\{g(s)\}^{p-1} g^{\prime}(s-t) d s, \\
& \bar{H}_{+}(g, t)=\int_{0}^{1-t}\{g(s)\}^{p-1} g^{\prime}(s+t) d s,
\end{aligned}
$$

we have

$$
G(g)_{i \pm}^{n}=H_{ \pm}\left(\frac{i}{k_{n}}, g\right)+\mathrm{O}_{u}\left(\sqrt{\Delta_{n}}\right), \quad \bar{G}(g)_{i \pm}^{n}=\bar{H}_{ \pm}\left(\frac{i}{k_{n}}, g\right)+\mathrm{O}_{u}\left(\sqrt{\Delta_{n}}\right) .
$$

Using $\left|G_{0}^{n}\right| \leq K$ and $X^{\prime}=M+X^{\prime \prime},(5.86),(5.87),(5.88)$ and (SN-2p), we deduce

$$
\eta(q, g)_{m}^{n}=\sigma_{T_{m}-} \rho(g)_{m-}^{n}+\sigma_{T_{m}} \rho(g)_{m+}^{n}+\bar{\rho}(g)_{m-}^{n}+\bar{\rho}(g)_{m+}^{n}+\mathrm{o}_{P u}(1)
$$

where

$$
\begin{gathered}
\rho(g)_{m-}^{n}=\frac{1}{\Delta_{n}^{1 / 4}} \sum_{i=0}^{k_{n}-3} H_{-}\left(\frac{i}{k_{n}}, g\right) \Delta_{I_{m}^{n}-i}^{n} W, \\
\rho(g)_{m+}^{n}=\frac{1}{\Delta_{n}^{1 / 4}} \sum_{i=2}^{k_{n}-1} H_{-}\left(\frac{i}{k_{n}}, g\right) \Delta_{I_{m}^{n}+i}^{n} W, \\
\bar{\rho}(g)_{m-}^{n}=-\frac{1}{\Delta_{n}^{1 / 4} k_{n}} \sum_{i=0}^{k_{n}-2} \bar{H}_{-}\left(\frac{i}{k_{n}}, g\right) \chi_{I_{m}^{n}-i}^{n}, \\
\bar{\rho}(g)_{m+}^{n}=-\frac{1}{\Delta_{n}^{1 / 4} k_{n}} \sum_{i=1}^{k_{n}-1} \bar{H}_{+}\left(\frac{i}{k_{n}}, g\right) \chi_{I_{m}^{n}+i}^{n} .
\end{gathered}
$$

(3) At this stage, we use the same ideas as in Lemma 5.1. We denote by $\rho_{m \pm}^{n}$ and $\bar{\rho}_{m \pm}^{n}$ the $d$-dimensional variables with components $\rho\left(g_{i}\right)_{m \pm}^{n}$ and $\bar{\rho}\left(g_{i}\right)_{m \pm}^{n}$. First we argue that $\omega^{(0)} \in \Omega^{(0)}$ fixed. Under $\mathbb{Q}=\mathbb{Q}\left(\omega^{(0)}, \cdot\right)$ the variables $\bar{\rho}_{m-}^{n}$ and $\bar{\rho}_{m+}^{n}$ are independent from each other and also when $m$ varies in $P_{p}^{\prime}$ as soon as $n$ is large enough [so that $\omega^{(0)} \in \Omega_{n}(t, q)$ ]. Moreover, they are 
sums, normalized by $1 / \Delta_{n}^{1 / 4} k_{n}$, of (approximately) $k_{n}$ centered independent variables with a bounded fourth moment, and their covariance matrices are (approximately again) $\alpha_{T_{m}-}^{2} / \theta$ and $\alpha_{T_{m}}^{2} / \theta$ times Riemann approximations of the integrals defining $\bar{\Psi}_{p-}$ and $\bar{\Psi}_{p+}$, respectively. Then we prove exactly as for (5.26) (only the finite-dimensional convergence is needed here) that under $\mathbb{Q}$,

$$
\left(\bar{\rho}_{m-}^{n}, \bar{\rho}_{m+}^{n}\right)_{m \in P_{q}^{\prime}} \stackrel{\mathcal{L}}{\longrightarrow}\left(\frac{\alpha_{T_{m}-}\left(\omega^{(0)}\right)}{\sqrt{\theta}} \bar{U}_{m-}, \frac{\alpha_{T_{m}}\left(\omega^{(0)}\right)}{\sqrt{\theta}} \bar{U}_{m+}\right)_{m \in P_{q}^{\prime}} .
$$

[In fact we prove the convergence in (5.90) for each $m$ first, and then we use the fact that the variables in the left-hand side are independent for different values of $m$, under $\mathbb{Q}$, and as soon as $\omega^{(0)} \in \Omega_{n}(t, q)$.]

Second, exactly as for (5.33) [or as above for (5.90)], we get

$$
\left(\rho_{m-}^{n}, \rho_{m+}^{n}\right)_{m \in P_{q}^{\prime}} \stackrel{\mathcal{L}}{\longrightarrow}\left(\sqrt{\theta} U_{m-}, \sqrt{\theta} U_{m+}\right)_{m \in P_{q}^{\prime}} \cdot
$$

Then $\left(U_{m \pm}, \bar{U}_{m \pm}\right)$ are as described after (4.5), and as in Steps 2 and 4 of the proof of Lemma 5.1 , we deduce from the convergences $(5.90)$ under $\mathbb{Q}\left(\omega^{(0)}, \cdot\right)$ and (5.91) under $\mathbb{P}^{(0)}$, and from (5.89), that $\left(\rho_{m-}^{n}, \rho_{m+}^{n}, \bar{\rho}_{m-}^{n}, \bar{\rho}_{m+}^{n}\right)_{m \in P_{q}^{\prime}}$ converges in law to $\left(\sqrt{\theta} U_{m-}, \sqrt{\theta} U_{m+}, \alpha_{T_{m}-} \bar{U}_{m-} / \sqrt{\theta}, \alpha_{T_{m}} \bar{U}_{m+} / \sqrt{\theta}\right)_{m \in P_{q}^{\prime}}$. This convergence in law is indeed a stable convergence by the same argument used to obtain a similar result in [15]. Finally by (5.85) and (5.89) and the definition of the stable convergence in law, we obtain the claim.

Proof of Proposition 5.12. With $\bar{g}_{l}(p)_{n}=\sum_{i=1}^{k_{n}}\left|g_{l}\left(i / k_{n}\right)\right|^{p}$, we have $\left|\bar{g}_{l}(p)_{n}-k_{n} \bar{g}_{l}(p)\right| \leq K$ by (2.10). Then, with the notation (5.84), a Taylor expansion and $\left|\Delta X_{T_{m}}\right| \leq K$ yield

$$
\begin{aligned}
& \left|\zeta\left(q, g_{l}\right)_{m}^{n}-p\left\{\Delta X_{T_{m}}\right\}^{p-1} \eta\left(q, g_{l}\right)_{m}^{n}\right| \\
& \quad \leq K \Delta_{n}^{1 / 4}\left(1+\sum_{j=1}^{k_{n}-1}\left(\left(\overline{Z^{\prime q}}\left(g_{l}\right)_{I_{m}^{n}-j+1}^{n}\right)^{p}+\left(\overline{Z^{\prime q}}\left(g_{l}\right)_{I_{m}^{n}-j+1}^{n}\right)^{2}\right)\right) .
\end{aligned}
$$

If we apply (5.86) we see that the expectation of the sum in the right-hand side above is bounded (recall $p>3$ ). Therefore Lemma 5.13 implies

$$
\left(\zeta\left(q, g_{l}\right)_{m}^{n}\right)_{1 \leq l \leq d, m \in P_{q}} \stackrel{\mathcal{L}-(s)}{\longrightarrow}\left(p\left\{\Delta X_{T_{m}}\right\}^{p-1} \eta_{m}\right)_{m \in P_{q}}
$$

and (5.83) readily follows.

5.15. The processes $\widetilde{V}^{*}\left(X^{\prime q}, g, p\right)^{n}$. The aim of this subsection is to prove the following proposition. 
Proposition 5.14. Under the same assumptions as before, and for all $\varepsilon>0$, we have

$$
\lim _{q \rightarrow \infty} \limsup _{n} \mathbb{P}\left(\left|\widetilde{V}^{*}\left(X^{\prime q}, g, p\right)_{t}^{n}\right|>\varepsilon\right)=0
$$

The proof is based on the following easy property ( $g$ is fixed throughout):

$$
\widetilde{V}^{*}\left(X^{\prime q}, g, p\right)_{t}^{n}=\frac{1}{\Delta_{n}^{1 / 4} k_{n}} \sum_{i=0}^{\left[t / \Delta_{n}\right]-k_{n}} \Gamma(q)_{i}^{n}+R(q)_{t}^{n},
$$

where

$$
\begin{gathered}
\Gamma(q)_{i}^{n}=\left|\overline{Z^{\prime q}}(g)_{i}^{n}\right|^{p}-\sum_{j=1}^{k_{n}-1}\left|g_{j}^{n}\right|^{p} \Delta_{i+j}^{n} \Sigma(q), \quad \Sigma(q)_{t}=\sum_{s \leq t}\left|\Delta X^{\prime}(q)_{s}\right|^{p}, \\
\left|R(q)_{t}^{n}\right| \leq \frac{K}{\Delta_{n}^{1 / 4}}\left(\Sigma(q)_{t}\left(\frac{\bar{g}(p)_{n}}{k_{n}}-\bar{g}(p)\right)+\Sigma(q)_{u_{n}}+\left(\Sigma(q)_{t}-\Sigma(q)_{t-2 u_{n}}\right)\right) .
\end{gathered}
$$

LEMMA 5.15. We can find a sequence $\eta_{q}$ going to 0 as $q \rightarrow \infty$, with the following property: for any $q \geq 1$ and $i \geq 1$ we have a decomposition $\Gamma(q)_{i}^{n}=\Gamma^{\prime}(q)_{i}^{n}+\Gamma^{\prime \prime}(q)_{i}^{n}$ where both $\Gamma^{\prime}(q)_{i}^{n}$ and $\Gamma^{\prime \prime}(q)_{i}^{n}$ are $\mathcal{F}_{i+k_{n}}^{n}$-measurable and

$$
\left.\begin{array}{l}
\mathbb{E}\left(\left|\Gamma^{\prime}(q)_{i}^{n}\right|\right) \leq K_{q} \Delta_{n}^{1 \wedge(p / 4)}+\eta_{q} \Delta_{n}^{3 / 4}, \\
\mathbb{E}\left(\Gamma^{\prime \prime}(q)_{i}^{n} \mid \mathcal{F}_{i}^{n}\right)=0, \\
\mathbb{E}\left(\left|\Gamma^{\prime \prime}(q)_{i}^{n}\right|^{2}\right) \leq K_{q} \Delta_{n}^{3 / 2}+\eta_{q} \Delta_{n} .
\end{array}\right\}
$$

Proof. (1) Let us fix $i, q$ and $n$ which will be left out in most notation below. We consider the filtration $\mathcal{F}_{t}^{\prime}=\mathcal{F}_{i \Delta_{n}+t}$, and associated with this filtration the Brownian motion $W_{t}^{\prime}=W_{i \Delta_{n}+t}-W_{i \Delta_{n}}^{\prime}$ and the Poisson random measure $\mu^{\prime}((0, t] \times A)=\underline{\mu}\left(\left(i \Delta_{n}, i \Delta_{n}+t\right] \times A\right)$ whose compensator is still $\underline{\nu}$. Recalling (5.80), we set $b_{t}^{\prime}=b_{i \Delta_{n}+t}^{q}$, and observe that $\left|b_{t}^{\prime}\right| \leq K q$ because $b_{t}$ is bounded and $\int_{\{z: \gamma(z)>1 / q\}}|\delta(t, z)| \lambda(d z) \leq q \int \gamma^{2}(z) \lambda(d z)$. With all this notation and (5.79), we have

$$
X_{i \Delta_{n}+t}^{\prime q}=X_{i \Delta_{n}}^{\prime q}+\int_{0}^{t} b_{s}^{\prime} d s+\int_{0}^{t} \sigma_{s}^{\prime} d W_{s}^{\prime}+\left(\delta^{\prime} 1_{\{\gamma \leq 1 / q\}}\right) \star\left(\underline{\mu}^{\prime}-\underline{\nu}\right)_{t} .
$$

Recalling $g_{n}$ in (5.4), we then set

$$
\begin{aligned}
Y_{t}= & \int_{0}^{t} b_{s}^{\prime} g_{n}(s) d s+\int_{0}^{t} \sigma_{s}^{\prime} g_{n}(s) d W_{s}^{\prime}+\left(\delta^{\prime} g_{n} 1_{\{\gamma \leq 1 / q\}}\right) \star\left(\underline{\mu}^{\prime}-\underline{\nu}\right) t \\
& -\sum_{j=1}^{\left[t / \Delta_{n}\right]} g_{j}^{\prime n} \chi_{i+j-1}^{n} .
\end{aligned}
$$


Then by (2.11) and (5.4) and (5.93), we see that $\left.\overline{Z^{\prime}(g}\right)_{i}^{n}=Y_{u_{n}}$. If we further set

$$
Y_{t}^{\prime}=\left(\left|\delta^{\prime} g_{n}\right|^{p} 1_{\{\gamma \leq 1 / q\}}\right) \star \underline{\mu}_{t}^{\prime},
$$

we obtain $Y_{u_{n}}^{\prime}=\sum_{j=1}^{k_{n}-1}\left|g_{j}^{n}\right|^{p} \Delta_{i+j}^{n} \Sigma(q)$. Hence $\Gamma(q)_{i}^{n}=\left|Y_{u_{n}}\right|^{p}-Y_{u_{n}}^{\prime}$.

For simplicity of notation we write $f(x)=|x|^{p}$ which is $C^{2}$ (recall $p>3$ ), and we associate the functions

$$
\begin{aligned}
& F(x, y)=f(x+y)-f(x)-f^{\prime}(x) y, \\
& G(x, y)=f(x+y)-f(x)-f(y), \\
& H(x, y)=F(x, y)-f(y),
\end{aligned}
$$

which clearly satisfy

$$
\left.\begin{array}{l}
|F(x, y)| \leq K\left(|y|^{p}+y^{2}|x|^{p-2}\right), \\
|G(x, y)| \leq K\left(|x||y|^{p-1}+|y||x|^{p-1}\right), \\
|H(x, y)| \leq K\left(|x||y|^{p-1}+y^{2}|x|^{p-2}\right) .
\end{array}\right\}
$$

Then we apply Itô's formula and use (5.93) to obtain

$$
\left|Y_{t}\right|^{p}-Y_{t}^{\prime}=A_{t}+A_{t}^{\prime}+N_{t}+N_{t}^{\prime}
$$

where

$$
\begin{aligned}
A_{t}= & \int_{0}^{t} a_{s} d s, \quad A_{t}^{\prime}=\sum_{j=1}^{\left[t / \Delta_{n}\right]} F\left(Y_{j \Delta_{n}-},-g_{j}^{\prime n} \chi_{i+j-1}^{n}\right), \\
a_{t}= & f^{\prime}\left(Y_{t}\right) g_{n}(t) b_{t}^{\prime}+\frac{1}{2} f^{\prime \prime}\left(Y_{t}\right) g_{n}(t)^{2} \sigma_{t}^{\prime 2} \\
& +\int_{\{z: \gamma(z) \leq 1 / q\}} H\left(Y_{t}, g_{n}(t) \delta^{\prime}(t, z)\right) \lambda(d z)
\end{aligned}
$$

and $N_{t}$ is a martingale with angle bracket $C=\langle N, N\rangle$ given by

$$
\begin{aligned}
C_{t} & =\int_{0}^{t} c_{s} d s \\
c_{t} & =f^{\prime}\left(Y_{t}\right)^{2} g_{n}(t)^{2} \sigma_{t}^{\prime 2}+\int_{\{z: \gamma(z) \leq 1 / q\}} G\left(Y_{t}, g_{n}(t) \delta^{\prime}(t, z)\right)^{2} \lambda(d z)
\end{aligned}
$$

and, finally,

$$
N_{t}^{\prime}=-\sum_{j=1}^{\left[t / \Delta_{n}\right]} f^{\prime}\left(Y_{j \Delta_{n}-}\right) g_{j}^{\prime n} \chi_{i+j-1}^{n}
$$


which is another martingale (because the $\chi_{t}$ 's are centered) with square bracket,

$$
C_{t}^{\prime}:=\left[N^{\prime}, N^{\prime}\right]_{t}=\sum_{j=1}^{\left[t / \Delta_{n}\right]} f^{\prime}\left(Y_{j \Delta_{n}-}\right)^{2}\left(g_{j}^{\prime n}\right)^{2}\left(\chi_{i+j-1}^{n}\right)^{2} .
$$

(2) The decomposition $\Gamma(q)_{i}^{n}=\Gamma^{\prime}(q)_{i}^{n}+\Gamma^{\prime \prime}(q)_{i}^{n}$ is given by

$$
\Gamma^{\prime}(q)_{i}^{n}=A_{u_{n}}+A_{u_{n}}^{\prime}, \quad \Gamma^{\prime \prime}(q)_{i}^{n}=N_{u_{n}}+N_{u_{n}}^{\prime} .
$$

The $\mathcal{F}_{i+k_{n}}^{n}$-measurability of $\Gamma^{\prime}(q)_{i}^{n}$ and $\Gamma^{\prime \prime}(q)_{i}^{n}$ is obvious, as is the second part of (5.92). The rest of (5.92) will readily follow if we can find a sequence $\eta_{q} \rightarrow 0$ such that

$$
\left.\begin{array}{l}
\mathbb{E}\left(\left|A_{u_{n}}\right|\right) \leq K_{q} \Delta_{n}^{1 \wedge(p / 4)}+\eta_{q} \Delta_{n}^{3 / 4}, \quad \mathbb{E}\left(\left|A_{u_{n}}^{\prime}\right|\right) \leq K_{q} \Delta_{n}, \\
\mathbb{E}\left(C_{u_{n}}\right) \leq K_{q} \Delta_{n}^{3 / 2}+\eta_{q} \Delta_{n}, \quad \mathbb{E}\left(C_{u_{n}}^{\prime}\right) \leq K_{q} \Delta_{n}^{3 / 2}+\eta_{q} \Delta_{n} .
\end{array}\right\}
$$

For this we need moment estimates for $Y_{t}$ as defined by (5.93). Recall $\left|b^{\prime}\right| \leq K q$ and $\left|\sigma^{\prime}\right| \leq K$ and $\left|g_{n}\right| \leq K$ and $|\delta(\cdot, z)| \leq \gamma(z)$ whereas $\eta_{q}^{\prime}=$ $\int_{\{z: \gamma(z) \leq 1 / q\}} \gamma(z)^{2} \lambda(d z)$ goes to 0 as $q \rightarrow \infty$. In view of (SN-2p) and since $\left|g_{j}^{\prime n}\right| \leq K \sqrt{\Delta_{n}}$, and using the Burkholder-Davis-Gundy inequality for the martingale which is the last term in (5.93), we see that for all $r \in(0,2 p]$,

$$
\mathbb{E}\left(\left|Y_{t}\right|^{r}\right) \leq K q^{r} t^{r}+K t^{r / 2}+K \eta_{q}^{\prime} t^{1 \wedge(r / 2)} .
$$

By $f(x)=|x|^{p}$ and (5.94), plus $p>3$, we see that $\left|a_{t}\right| \leq K\left(q\left|Y_{t}\right|^{p-1}+\left|Y_{t}\right|^{p-2}+\right.$ $\left.\eta_{q}^{\prime}\left|Y_{t}\right|\right)$. Therefore (5.96) yields $\mathbb{E}\left(\left|a_{t}\right|\right) \leq K q^{p} t^{1 \wedge(p / 2-1)}+K \eta_{q}^{\prime} t^{1 / 2}$. In a similar way $c_{t} \leq K\left|Y_{t}\right|^{2 p-2}+K \eta_{q}^{\prime} Y_{t}^{2}$; hence $\mathbb{E}\left(c_{t}\right) \leq K\left(q^{2 p-2} t^{2}+\eta_{q}^{\prime} t\right)$. Then the estimate for $A_{u_{n}}$ and $C_{u_{n}}$ in (5.95) follows upon taking $\eta_{q}=K \eta_{q}^{\prime}$ for a $K$ large enough.

For the same reasons, plus $(\mathrm{SN}-2 p)$, the $j$ th summand in the definition of $A_{t}^{\prime}$ has an expectation smaller than $K \Delta_{n}^{p / 2}+K \Delta_{n}\left(q^{p-2}\left(j \Delta_{n}\right)^{p-2}+\right.$ $\left.\left(j \Delta_{n}\right)^{p / 2-1}+\eta_{q}^{\prime}\left(j \Delta_{n}\right)\right)$ whereas the $j$ th summand in the expression for $C_{t}^{\prime}$ has an expectation smaller than $K \Delta_{n}\left(q^{2 p-2}\left(j \Delta_{n}\right)^{2 p-2}+\left(j \Delta_{n}\right)^{p-1}+\eta_{q}^{\prime}\left(j \Delta_{n}\right)\right)$. The two other estimates in (5.95) follow.

Proof of Proposition 5.14. In view of (2.10) and of the fact that $\mathbb{E}\left(\Sigma(q)_{s+u}-\Sigma(q)_{s}\right) \leq K_{q} u$, we deduce that $R(q)_{t}^{n} \stackrel{\mathbb{P}}{\longrightarrow} 0$ for all $q$. Hence it remains to prove that

$$
\lim _{q \rightarrow \infty} \limsup _{n} \mathbb{P}\left(\left.\frac{1}{\Delta_{n}^{1 / 4} k_{n}}\right|^{\left[t / \Delta_{n}\right]-k_{n}} \sum_{i=0} \Gamma(q)_{i}^{n} \mid>\varepsilon\right)=0 .
$$


We set, with $t$ fixed and the notation of the previous lemma,

$$
L^{\prime}(q)_{n}=\frac{1}{\Delta_{n}^{1 / 4} k_{n}} \sum_{i=0}^{\left[t / \Delta_{n}\right]-k_{n}} \Gamma^{\prime}(q)_{i}^{n}, \quad L^{\prime \prime}(q)_{n}=\frac{1}{\Delta_{n}^{1 / 4} k_{n}} \sum_{i=0}^{\left[t / \Delta_{n}\right]-k_{n}} \Gamma^{\prime \prime}(q)_{i}^{n} .
$$

The first property in (5.92) yields $\mathbb{E}\left(\left|L^{\prime}(q)_{n}\right|\right) \leq K_{q} \Delta_{n}^{(1 / 4) \wedge(p / 4-3 / 4)}+\eta_{q}$; hence since $p>3$,

$$
\lim _{q \rightarrow \infty} \limsup _{n} \mathbb{E}\left(\left|L_{n}^{\prime}\right|\right)=0 .
$$

Next, the properties of $\Gamma^{\prime \prime}(q)_{i}^{n}$ in the Lemma 5.15 imply that $\left|\mathbb{E}\left(\Gamma^{\prime \prime}(q)_{i}^{n} \Gamma^{\prime \prime}(q)_{j}^{n}\right)\right|$ vanishes when $|j-i|>k_{n}$, and, otherwise, is smaller than $K_{q} \Delta_{n}^{3 / 2}+\eta_{q} \Delta_{n}$. Hence $\mathbb{E}\left(\left(L_{n}^{\prime \prime}\right)^{2}\right) \leq K_{q} \Delta_{n}^{1 / 2}+\eta_{q}$, which yields

$$
\lim _{q \rightarrow \infty} \limsup _{n} \mathbb{E}\left(\left|L_{n}^{\prime \prime}\right|^{2}\right)=0 .
$$

Putting these two results together immediately yields (5.97).

5.16. Proof of Theorem 4.4. We start with the first claim, which easily follows from what precedes. The family $\left(g_{l}\right)$ of weight functions is fixed. Since $U(p, q)_{t} \stackrel{\mathbb{P}}{\longrightarrow} U(p)_{t}$ as $q \rightarrow \infty$, the result is a trivial consequence of (5.82) and Propositions 5.12 and 5.14 .

Next, we show that the second claim can be reduced to the first claim. We take $p \geq 4$, an even integer, and it is enough to prove that $\frac{1}{\Delta_{n}^{1 / 4} k_{n}}\left(\bar{V}^{*}(g, p)^{n}-\right.$ $\left.\widetilde{V}^{*}(g, p)^{n}\right) \stackrel{\text { u.c.p. }}{\longrightarrow} 0$ for any weight function $g$. To see this we observe that the difference $\bar{V}^{*}(g, p)_{t}^{n}-\widetilde{V}^{*}(g, p)_{t}^{n}$ is a linear combination of the processes (we omit to mention the function $g$ below)

$$
\frac{1}{\Delta_{n}^{1 / 4} k_{n}} \sum_{i=0}^{\left[t / \Delta_{n}\right]-k_{n}}\left(\bar{Z}_{i}^{n}\right)^{p-2 r}\left(\widehat{Z}_{i}^{n}\right)^{r}
$$

for $r=1, \ldots, p / 2$. So it enough to prove that, for some $\rho>3 / 4$ and all $r=1, \ldots, p / 2$,

$$
\mathbb{E}\left(\left(\bar{Z}_{i}^{n}\right)^{p-2 r}\left(\widehat{Z}_{i}^{n}\right)^{r}\right) \leq K \Delta_{n}^{\rho} .
$$

Hypothesis (SK) yields $\mathbb{E}\left(\left|\Delta_{i}^{n} X\right|^{v} \mid \mathcal{F}_{i-1}^{n}\right) \leq K \Delta_{n}^{(v \wedge 2) / 2}$ and (SN-4p) holds, so when $v \geq 1$ we have

$$
\left.\begin{array}{l}
\mathbb{E}\left(\left|\bar{X}_{i}^{n}\right|^{v} \mid \mathcal{F}_{i}^{n}\right) \leq K_{v} \Delta_{n}^{(v / 4) \wedge(1 / 2)}, \quad \mathbb{E}\left(\left|\widehat{X}_{i}^{n}\right|^{v} \mid \mathcal{F}_{i}^{n}\right) \leq K_{v} \Delta_{n}^{1+v / 2}, \\
v \leq 2 p \Rightarrow \mathbb{E}\left(\left|\sum_{j=1}^{k_{n}}\left(g_{j}^{n}\right)^{2} \Delta_{i+j}^{n} X \Delta_{i+j}^{n} \chi\right|^{v} \mid \mathcal{F}_{i}^{n}\right) \leq K_{v} \Delta_{n}^{v / 2+(v / 2) \wedge 1} .
\end{array}\right\}
$$


Now $\left(\bar{Z}_{i}^{n}\right)^{p-2 r}\left(\widehat{Z}_{i}^{n}\right)^{r}$ is a linear combination of terms of the form

$$
a(u, v, w, s, t)_{i}^{n}=\left(\bar{X}_{i}^{n}\right)^{u}\left(\widehat{X}_{i}^{n}\right)^{v}\left(\bar{\chi}_{i}^{n}\right)^{w}\left(\widehat{\chi}_{i}^{n}\right)^{s}\left(\sum_{j=1}^{k_{n}}\left(g_{j}^{n}\right)^{2} \Delta_{i+j}^{n} X \Delta_{i+j}^{n} \chi\right)^{t},
$$

where $u, v, w, s, t$ are integers with $u+w=p-2 r$ and $v+s+t=r$. Using Hölder's inequality, and taking advantage of (5.99) and of $\mathbb{E}\left(\left|\bar{\chi}_{i}^{n}\right|^{l}\right) \leq K_{r} \Delta_{n}^{l / 4}$ and $\mathbb{E}\left(\left|\widehat{\chi}_{i}^{n}\right|^{l}\right) \leq K_{r} \Delta_{n}^{l / 2}$, we see that for all $u^{\prime}, v^{\prime}, w^{\prime}, s^{\prime}, t^{\prime} \geq 0$ such that $u^{\prime}+$ $v^{\prime}+w^{\prime}+s^{\prime}+t^{\prime}=1$ and $u^{\prime}=0$ (resp., $v^{\prime}=0, w^{\prime}=0, s^{\prime}=0, t^{\prime}=0$ ) if and only if $u=0$ (resp., $v=0, w=0, s=0, t=0$ ), and also $\frac{w}{w^{\prime}} \vee \frac{2 s}{s^{\prime}} \vee \frac{t}{t^{\prime}} \leq 2 p$ (which is possible because $w+2 s+t \leq p)$, we have $\mathbb{E}\left(\left|a(u, v, w, s, t)_{i}^{n}\right|\right) \leq K \Delta_{n}^{\rho}$ where

$$
\begin{aligned}
\rho & =\frac{u}{4} \wedge \frac{u^{\prime}}{2}+v^{\prime} 1_{v>0}+\frac{v}{2}+\frac{w}{4}+\frac{s}{2}+\frac{t}{2}+t^{\prime} \wedge \frac{t}{2} \\
& =\frac{r}{2}+\frac{w}{4}+\frac{u}{4} \wedge \frac{u^{\prime}}{2}+v^{\prime} 1_{v>0}+t^{\prime} \wedge \frac{t}{2} .
\end{aligned}
$$

Then $\rho>3 / 4$ as soon as $r \geq 2$, or $r=1$ and $w \geq 1$. The only other case is $r=1$ and $w=0$, so $u=p-2 \geq 2$ and we have

$$
\rho=\frac{1}{2}+\frac{u^{\prime}}{2}+v^{\prime} 1_{v>0}+t^{\prime} \wedge \frac{t}{2} .
$$

Then we have three sub-cases:

(1) $v=1$, hence $t=t^{\prime}=s=s^{\prime}=w^{\prime}=0$ and $\rho=\frac{1+u^{\prime}}{2}+v^{\prime}$ with the condition $u^{\prime}+v^{\prime}=1$, so $u^{\prime}=v^{\prime}=1 / 2$ yields $\rho>3 / 4$;

(2) $s=1$, hence $t=t^{\prime}=v=v^{\prime}=w^{\prime}=0$ and $\rho=\frac{1+u^{\prime}}{2}$ with the conditions $u^{\prime}+s^{\prime}=1$ and $s^{\prime} p \geq 1$, so $s^{\prime}=1 / 3$ yields $\rho>3 / 4$;

(3) $t=1$, hence $v=v^{\prime}=s=s^{\prime}=w^{\prime}=0$ and $\rho=\frac{1}{2}+\frac{u^{\prime}}{2}+t^{\prime} \wedge \frac{1}{2}$ with the condition $u^{\prime}+t^{\prime} 11$ and $2 t^{\prime} p \geq 1$, so $u^{\prime}=t^{\prime}=1 / 2$ yield $\rho>3 / 4$.

Hence in all cases (5.98) holds with some $\rho>3 / 4$, and the proof is complete.

5.17. Proof of Theorem 4.6. Here again the proof will be divided into several steps, and before proceeding we observe two preliminary facts. First, that $\bar{\mu}_{4}(g, g ; \eta, \zeta)$ takes the form $(4.9)$ results from a tedious but elementary calculation. Second, by localization we may assume (SN-4) and Hypothesis $(\mathrm{SH})$.

We omit the mention of the function $g$ in $\bar{Y}_{i}^{n}$ and $\widehat{Y}_{i}^{n}$. We generally use the notation of the proof of Theorem 4.4, and in particular the stopping times $T(q, m)$ and $T_{m}$ introduced in Section 5.13, the processes of (5.79), the sets $\Omega_{n}(t, q)$ satisfying (5.81) and the (random) integers $I_{m}^{n}$. In the sequel, we will vary the process $X$ (but not the noise process $\chi$ ), so the process $\bar{V}^{n}$ of (4.7) will be denoted by $\bar{V}(X)^{n}$. We also write $U^{\prime}(\sigma)_{t}$ and $U(2, \sigma, \delta)_{t}$ for the two 
terms in (4.8), and $\bar{U}(\sigma, \delta)_{t}$ for their sum, to emphasize their dependency on the process $\sigma$ and the function $\delta$ (through the jumps of $X$, for the latter).

Step 1. In this step we prove the result when, in addition to Hypothesis (SH), we have

$$
\left.\begin{array}{l}
\gamma(z) \leq 1 / q \Rightarrow \delta(\omega, t, z)=0 \\
b_{s}^{\prime}=b_{s}-\int \delta(t, x) 1_{\{|\delta(y, z)| \leq 1\}} \lambda(d z)=\sum_{r \geq 1} b_{S_{r}} 1_{\left[S_{r}, S_{r+1}\right)}(t), \\
\sigma_{s}=\sum_{r \geq 1} \sigma_{S_{r}} 1_{\left[S_{r}, S_{r+1}\right)}(t),
\end{array}\right\}
$$

for some $q \geq 1$ and a sequence of stopping times $S_{r}$, increasing to $\infty$ and with $S_{0}=0$.

(1) Under (5.100) and (5.101) we have $X_{t}^{q}=\sum_{s \leq t} \Delta X_{s}$, and $X^{\prime}=X^{\prime q}$ is the continuous process given by the right-hand side of (2.14) with $b^{\prime}$ instead of $b$. Similarly to $(5.82)$, we have on $\Omega_{n}(t, q)$,

$$
\begin{aligned}
\bar{V}(X)_{t}^{n} & =\bar{V}\left(X^{\prime}\right)_{t}^{n}+Y_{t}^{n}-\frac{1}{2} Y_{t}^{\prime n}, \\
Y_{t}^{n} & =\sum_{m \in P_{q}: T_{m} \leq t} \zeta_{m}^{n}, \quad Y_{t}^{\prime n}=\sum_{m \in P_{q}: T_{m} \leq t} \zeta_{m}^{\prime n}, \\
\zeta_{m}^{n} & =\frac{1}{\Delta_{n}^{1 / 4} k_{n}}\left(\sum _ { j = 1 } ^ { k _ { n } - 1 } \left(\overline{\left(X^{\prime}+\chi\right)_{I_{m}^{n}+1-j}^{n}}+\left.g_{j}^{n} \Delta X_{T_{m}}\right|^{2}\right.\right. \\
& \left.-\left|\overline{\left(X^{\prime}+\chi\right)_{I_{m}^{n}+1-j}^{n}}\right|^{2}-\left|g_{j}^{n} \Delta X_{T_{m}}\right|^{2}\right) \\
\left.\quad+\left(\bar{g}(2)_{n}-k_{n} \bar{g}(2)\right)\left|\Delta X_{T_{m}}\right|^{2}\right), & \\
\zeta_{m}^{\prime n} & =\frac{1}{\Delta_{n}^{1 / 4} k_{n}} \sum_{j=1}^{k_{n}}\left(g_{j}^{\prime n}\right)^{2}\left(\left(\Delta X_{T_{m}}\right)^{2}+2 \Delta X_{T_{m}} \Delta_{I_{m}^{n}+1-j}^{n}\left(X^{\prime}+\chi\right)\right) .
\end{aligned}
$$

Let $\left(\mathcal{H}_{t}\right)$ be the filtration defined in the proof of Lemma 5.13 and associated with our $q$. The same argument used in that lemma shows $\mathbb{E}\left(\mid \Delta_{I_{m}^{n}+1-j}^{n}\left(X^{\prime}+\right.\right.$ $\left.\chi)|| \mathcal{H}_{0}\right) \leq K$ whereas $\left|\Delta X_{T_{m}}\right| \leq K$ by Hypothesis (SH). It follows that $\mathbb{E}\left(\left|\zeta_{m}^{\prime n}\right|\right) \leq K \Delta_{n}^{3 / 4} ;$ hence

$$
Y_{t}^{\prime n} \stackrel{\mathbb{P}}{\longrightarrow} 0 .
$$

(2) Next we prove the (functional) stable convergence $\bar{V}\left(X^{\prime}\right)^{n} \stackrel{\mathcal{L}-(s)}{\longrightarrow} U^{\prime}(\sigma)$. This looks the same as Theorem 4.1 for $p=2$, however we do not have Hypothesis (K) here. Now a look at the proof of this theorem shows that Hypothesis $(\mathrm{K})$ [instead of Hypothesis $(\mathrm{H})$ ] is used in two places only, namely 
for (5.63) for $k=2$, and in Lemma 5.8. Here, the proof of Lemma 5.8 proceeds in an obvious way under (5.101), and we are left to show that (5.63) holds when $k=2$.

The variable $\Delta_{n}^{3 / 4-p / 2} U_{t}^{n, 2}$ for $p=2$ is the sum $\sum_{i=1}^{i_{n}(m, t)} \sum_{j=0}^{m k_{n}-1} \theta_{i, j}^{n}$, where

$$
\theta_{i, j}^{n}=\Delta_{n}^{1 / 4} \mathbb{E}\left(\phi\left(X^{\prime}+\chi, g, 2\right)_{I(m, n, i), j}-\phi(g, 2)_{I(m, n, i)+j} \mid \mathcal{F}(m)_{i-1}^{n}\right) .
$$

Let $J_{n}$ be the set of all $i$ such that $(i-1)(m+1) u_{n}<S_{r} \leq i m u_{n}$ for some $r \geq 1$ (the indices of those "big blocks" that contain at least one $S_{r}$ ), and consider the two processes

$$
A_{t}^{n}=\sum_{i \in\left\{1, \ldots, i_{n}(m, t)\right\} \cap J_{n}} \sum_{j=0}^{m k_{n}-1} \theta_{i, j}^{n}, \quad A_{t}^{\prime n}=\sum_{i \in\left\{1, \ldots, i_{n}(m, t)\right\} \cap J_{n}^{c}} \sum_{j=0}^{m k_{n}-1} \theta_{i, j}^{n} .
$$

Applying (5.45) with $u=1$ [recall (SN-4)], we obtain $\mathbb{E}\left(\left|\theta_{i, j}^{n}\right|\right) \leq K \Delta_{n}^{3 / 4}$. Therefore $\mathbb{E}\left(\sup _{s \leq t \wedge S_{r}}\left|A_{s}^{n}\right|\right)$ is obviously smaller than $K r \Delta_{n}^{1 / 4}$ and, since $S_{r} \rightarrow \infty$ as $r \rightarrow \infty$, we deduce $A^{n} \stackrel{\text { u.c.p. }}{\longrightarrow} 0$, and it remains to prove the same for $A^{\prime n}$.

For this, and reproducing the proof of Lemma 5.7, we observe that Hypothesis $(\mathrm{K})$ comes in only to decompose the variables $\bar{\lambda}_{i+j}^{n}$ as $\xi_{i, j}^{n}+\xi_{i, j}^{\prime n}$. We easily deduce from (5.101) that when $i \notin J_{n}$ such a decomposition holds with $\xi_{i, j}^{n}=0$ and $\xi_{i, j}^{\prime n}=b_{i \Delta_{n}}^{\prime} \Delta_{n}$. Then the original proof goes through to show that $A^{\prime \prime} \stackrel{\text { u.c.p. }}{\longrightarrow} 0$, and thus (5.63) for $k=2$ holds here.

(3) We have $\bar{V}\left(X^{\prime}\right)^{n} \stackrel{\mathcal{L}-(s)}{\longrightarrow} U^{\prime}(\sigma)$ from what precedes, and this gives the result (functional stable convergence in law) when $X$ is continuous, in addition to satisfying (5.101). When $X$ has jumps, the proof of Proposition 5.12 is valid when $p=2$ (it only supposes the $C^{2}$ property of $x \mapsto|x|^{p}$ ), so $Y_{t}^{n} \stackrel{\mathcal{L}-(s)}{\longrightarrow} U(2, \sigma, \delta)_{t}$ (for $t$ fixed, not functional convergence).

Now, exactly as in the proof of Lemma 5.8 in [13], one can show that we have the joint stable convergence in law in Proposition 5.10 and Lemma 5.13 which results in the joint convergence

$$
\left(\bar{V}\left(X^{\prime}\right)_{t}^{n}, Y_{t}^{n}\right) \stackrel{\mathcal{L}-(s)}{\longrightarrow}\left(U^{\prime}(\sigma)_{t}, U(2, \sigma, \delta)_{t}\right)
$$

Then we easily deduce from $(5.81),(5.102)$ and $(5.103)$ that $\bar{V}(X)_{t}^{n} \stackrel{\mathcal{L}-(s)}{\longrightarrow}$ $\bar{U}(\sigma, \delta)_{t}$.

Step 2. We turn to the general case, and we begin by constructing an approximation of $X$ satisfying (5.100) and (5.101).

For $q \geq 1$ we recall the process $b^{q}$ of (5.80). If further $r \geq 1$ we denote by $S(q, r)_{r}$ the strictly increasing rearrangement of the points in the set $\left\{k 2^{-r}: k \geq 0\right\} \cup\{T(q, m): m \geq 1\}$. By a classical density argument there are 
adapted processes $b(q, r)$ and $\sigma(q, r)$ with the following properties: they are bounded by the same bounds as $b^{q}$ and $\sigma$, respectively, constant over each interval $\left[(k-1) 2^{-r}, k 2^{-r}\right)$ for $b(q, r)$ and each interval $\left[S(q, r)_{k-1}, S(q, r)_{q}\right)$ for $\sigma$ and such that for all $q, m \geq 1$ and $t \geq 0$,

$$
r \rightarrow \infty \Rightarrow\left\{\begin{array}{c}
\varepsilon(q, r)_{t}=\mathbb{E}\left(\int _ { 0 } ^ { t } \left(\left|b(q, r)_{s}-b_{s}^{q}\right|^{2}\right.\right. \\
\left.\left.+\left|\sigma(q, r)_{s}-\sigma_{s}\right|^{2}\right) d s\right) \rightarrow 0 \\
\sigma(q, r)_{T(q, m)}=\sigma_{T(q, m)}, \\
\sigma(q, r)_{T(q, m)-} \rightarrow \sigma_{T(q, m)-},
\end{array}\right.
$$

(we use here the càdlàg property of $\sigma$ ). Next, we introduce the following family of processes:

$$
\left.\begin{array}{rl}
X(q, r)_{t} & =X_{0}+\int_{0}^{t} b(q, r)_{s} d s+\int_{0}^{t} \sigma(q, r)_{s} d W_{s}+\left(\delta 1_{\{\gamma>1 / q\}}\right) * \underline{\mu}, \\
X^{\prime}(q, r)_{t} & =X_{t}-X(q, r)_{t} \\
& =\int_{0}^{t}\left(b_{s}^{q}-b(q, r)_{s}\right) d s+\int_{0}^{t}\left(\sigma_{s}-\sigma(q, r)_{s}\right) d W_{s}+M_{t}^{q}
\end{array}\right\}
$$

[here $M^{q}$ is given by (5.79)]. Finally, another notation will be

$$
\begin{aligned}
\varepsilon(q, r)_{i}^{n} & =\mathbb{E}\left(\int_{i \Delta_{n}}^{i \Delta_{n}+u_{n}}\left(\left|b(q, r)_{s}-b_{s}^{q}\right|^{2}+\left|\sigma(q, r)_{s}-\sigma_{s}\right|^{2}\right) d s\right), \\
\varepsilon_{q} & =\int_{\{z: \gamma(z) \leq 1 / q\}} \gamma(z)^{2} \lambda(d z) .
\end{aligned}
$$

By construction $X(q, r)$ satisfies (5.100) and (5.101), so Step 1 gives

$$
\bar{V}(X(q, r))_{t}^{n} \stackrel{\mathcal{L}-(s)}{\longrightarrow} \bar{U}(\sigma(q, r), \delta(q))_{t}
$$

for any $t$ and $q, r \geq 1$, and where $\delta(q)(\omega, t, z)=\delta(\omega, t, z) 1_{\{\gamma(z)>1 / q\}}$, and the convergence even holds in the functional sense when $X$ is continuous.

Note that, since $\sigma$ and $\sigma(q, r)$ and $\alpha$ are uniformly bounded and the function $\bar{\mu}_{4}$ in (4.9) is locally Lipschitz in $(\eta, \zeta)$, we have

$$
\mathbb{E}\left(\sup _{s \leq t}\left|U^{\prime}(\sigma)_{s}-U^{\prime}(\sigma(q, r))_{s}\right|^{2}\right) \leq K \mathbb{E}\left(\int_{0}^{t}\left|\sigma_{s}-\sigma(q, r)_{s}\right|^{2} d s\right) \leq K \varepsilon(q, r)_{t} .
$$

On the other hand, since $\delta(q)$ is bounded, it follows from (4.6) that

$$
\begin{aligned}
& \mathbb{E}\left(\sup _{s \leq t}\left|U(2, \sigma, \delta(q))_{s}-U(2, \sigma(q, r), \delta(q))_{s}\right|^{2}\right) \\
& \quad \leq K \mathbb{E}\left(\sum_{m \geq 1}\left|\sigma_{T(q, m)-}-\sigma(q, r)_{T(q, m)-}\right|^{2} 1_{\{T(q, m) \leq t\}}\right),
\end{aligned}
$$


which goes to 0 as $r \rightarrow \infty$ by (5.104). Furthermore,

$$
\mathbb{E}\left(\sup _{s \leq t}\left|U(2, \sigma, \delta(q))_{s}-U(2, \sigma, \delta)_{s}\right|^{2}\right) \leq K \mathbb{E}\left(\sum_{s \leq t}\left|\Delta X_{s}\right|^{2} 1_{\left\{\left|\Delta X_{s}\right| \leq 1 / q\right\}}\right),
$$

which goes to 0 as $q \rightarrow \infty$. Summarizing those results, we end up with

$$
\lim _{q \rightarrow \infty} \limsup _{r \rightarrow \infty} \mathbb{E}\left(\sup _{s \leq t}\left|\bar{U}(\sigma, \delta)_{s}-\bar{U}(\sigma(q, r), \delta(q))_{s}\right|^{2}\right)=0 .
$$

Therefore, in order to get our theorem it remains to prove that for all $t, \eta>0$ we have, where $\mathrm{C}$ refers to the case, $X$ is continuous and $\mathrm{D}$ to the general (discontinuous) case,

$$
\left.\begin{array}{l}
\text { C: } \lim _{q \rightarrow \infty} \limsup _{r \rightarrow \infty} \limsup _{n \rightarrow \infty} \mathbb{P}\left(\sup _{s \leq t}\left|\bar{V}(X(q, r))_{s}^{n}-\bar{V}(X)_{s}^{n}\right|>\eta\right)=0, \\
\text { D: } \lim _{q \rightarrow \infty} \limsup _{r \rightarrow \infty} \limsup _{n \rightarrow \infty} \mathbb{P}\left(\left|\bar{V}(X(q, r))_{t}^{n}-\bar{V}(X)_{t}^{n}\right|>\eta\right)=0 .
\end{array}\right\}
$$

Step 3. If $Z(q, r)=X(q, r)+\chi$, we have

$$
\begin{gathered}
\phi(Z, g, 2)_{i}^{n}-\phi(Z(q, r), g, 2)_{i}^{n} \\
=\left(\bar{X}_{i}^{n}\right)^{2}-\left(\overline{X(q, r)_{i}^{n}}\right)^{2}+2 \bar{\chi}_{i}^{n}\left(\bar{X}_{i}^{n}-\overline{X(q, r)_{i}^{n}}\right)-\frac{1}{2} v_{i}^{n}, \\
v_{i}^{n}=\sum_{j=1}^{k_{n}}\left(g_{j}^{\prime n}\right)^{2}\left(\left(\Delta_{i+j}^{n} X\right)^{2}-\left(\Delta_{i+j}^{n} X(q, r)\right)^{2}\right. \\
\left.+2 \Delta_{i+j}^{n} \chi\left(\Delta_{i+j}^{n} X-\Delta_{i+j}^{n} X(q, r)\right)\right) .
\end{gathered}
$$

Therefore

$$
\bar{V}(X)_{t}^{n}-\bar{V}(X(q, r))_{t}^{n}=G^{1}(q, r)_{t}^{n}+G^{2}(q, r)_{t}^{n}-\frac{1}{2} V_{t}^{n},
$$

where

$$
\begin{aligned}
V_{t}^{n}= & \frac{1}{k_{n} \Delta_{n}^{1 / 4}} \sum_{i=0}^{\left[t / \Delta_{n}\right]-k_{n}} v_{i}^{n}, \\
G^{1}(q, r)_{t}^{n}= & \frac{1}{\Delta_{n}^{1 / 4}}\left(\frac{1}{k_{n}} \sum_{i=0}^{\left[t / \Delta_{n}\right]-k_{n}}\left(\left(\bar{X}_{i}^{n}\right)^{2}-\left(\overline{X(q, r)_{i}^{n}}\right)^{2}\right)\right. \\
& \left.-\bar{g}(2)\left([X, X]_{t}-[X(q, r), X(q, r)]_{t}\right)\right), \\
G^{2}(q, r)_{t}^{n}= & \frac{2}{k_{n} \Delta_{n}^{1 / 4}} \sum_{i=0}^{\left[t / \Delta_{n}\right]-k_{n}} \bar{\chi}_{i}^{n}\left(\bar{X}_{i}^{n}-\overline{X(q, r}_{i}^{n}\right) .
\end{aligned}
$$


We obviously have $\mathbb{E}\left(\left|v_{i}^{n}\right|\right) \leq K \Delta_{n}$, so $V^{n} \stackrel{\text { u.c.p. }}{\longrightarrow} 0$. Therefore, instead of (5.106), we are left to prove for $l=1,2$,

$$
\left.\begin{array}{l}
\text { C: } \lim _{q \rightarrow \infty} \limsup _{r \rightarrow \infty} \limsup _{n \rightarrow \infty} \mathbb{P}\left(\sup _{s \leq t}\left|G^{l}(q, r)_{s}^{n}\right|>\eta\right)=0, \\
\text { D: } \lim _{q \rightarrow \infty} \limsup _{r \rightarrow \infty} \limsup _{n \rightarrow \infty} \mathbb{P}\left(\left|G^{l}(q, r)_{t}^{n}\right|>\eta\right)=0 .
\end{array}\right\}
$$

Step 4 . We begin by proving (5.107) for $l=2$. We split the sum in the definition of $G^{2}(q, r)_{t}^{n}$ into two parts: $G^{3}(q, r)_{t}^{n}$ is the sum over those $i$ 's such that the fractional part of $i / 2 k_{n}$ is in $[0,1 / 2)$, and $G^{4}(q, r)_{t}^{n}$ which is the sum when the fractional part is in $[1 / 2,1)$, so it enough to show $(5.107)$ for $l=3$ and $l=4$, and we will do it for $l=3$ only. We have

$$
\left.\begin{array}{l}
G^{3}(q, r)_{t}^{n}=\sum_{j=0}^{J_{n}+1} \zeta(q, r)_{i}^{n}, \\
\zeta(q, r)_{j}^{n}=\frac{2}{k_{n} \Delta_{n}^{1 / 4}} \sum_{i=2 j k_{n}}^{\left(2 j k_{n}+k_{n}-1\right) \wedge\left(\left[t / \Delta_{n}\right]-k_{n}\right)} \bar{\chi}_{i}^{n}\left(\bar{X}_{i}^{n}-\overline{X(q, r)_{i}^{n}}\right),
\end{array}\right\}
$$

where $J_{n}$ is the integer part of $\left(\left[t / \Delta_{n}\right]+1-2 k_{n}\right) / 2 k_{n}\left[J_{n}\right.$ depends on $t$, and all $\zeta(q, r)_{j}^{n}$ have $k_{n}$ summands, except the $J_{n}$ th one which may have less]. Note that $\zeta(q, r)_{j}^{n}$ is $\mathcal{F}_{2(j+1) k_{n}}^{n}$-measurable, and by successive conditioning we have $\mathbb{E}\left(\zeta(q, r)_{j}^{n} \mid \mathcal{F}_{2 j k_{n}}^{n}\right)=0$. Therefore by a martingale argument (5.107) will follow if we prove

$$
\lim _{q \rightarrow \infty} \limsup _{r \rightarrow \infty} \limsup _{n \rightarrow \infty} \mathbb{E}\left(\sum_{j=0}^{J(n, t)}\left|\zeta(q, r)_{j}^{n}\right|^{2}\right)=0 .
$$

Now, recall (5.105) and (5.100). Then, by (5.4) and standard estimates, plus (5.104) and the Cauchy-Schwarz inequality, plus (5.3) and successive conditioning, we get

$$
\mathbb{E}\left(\left(\bar{\chi}_{i}^{n}\right)^{2}\left(\bar{X}_{i}^{n}-\overline{X(q, r)}_{i}^{n}\right)^{2}\right) \leq K \Delta_{n}^{1 / 2}\left(\varepsilon(q, r)_{i}^{n}+u_{n} \varepsilon_{q}\right)
$$

and so the expectation in (5.108) is smaller than $K\left(\varepsilon(q, r)_{t}+\varepsilon_{q}\right)$. Hence (5.108) holds.

Step 5. Now we turn to $l=1$ in (5.107). We write $G^{1}(q, r)_{t}^{n}=G^{5}(q, r)_{t}^{n}+$ $G^{6}(q, r)_{t}^{n}$ where, with the notation $A(q, r)=[X, X]-[X(q, r), X(q, r)]$,

$$
\left.\begin{array}{rl}
G^{5}(q, r)_{t}^{n}= & \sum_{i=0}^{\left[t / \Delta_{n}\right]-k_{n}} \vartheta(q, r)_{i}^{n}, \\
\vartheta(q, r)_{i}^{n}=\frac{1}{k_{n} \Delta_{n}^{1 / 4}}\left(\left(\bar{X}_{i}^{n}\right)^{2}-\left(\overline{X(q, r)_{i}^{n}}\right)^{2}\right. \\
\left.\quad-\int_{i \Delta_{n}}^{i \Delta_{n}+u_{n}} g_{n}\left(s-i \Delta_{n}\right)^{2} d A(q, r)_{s}\right),
\end{array}\right\}
$$




$$
\begin{array}{r}
G^{6}(q, r)_{t}^{n}=\frac{1}{\Delta_{n}^{1 / 4}}\left(\frac{1}{k_{n}} \sum_{i=0}^{\left[t / \Delta_{n}\right]-k_{n}} \int_{i \Delta_{n}}^{i \Delta_{n}+u_{n}} g_{n}\left(s-i \Delta_{n}\right)^{2} d A(q, r)_{s}\right. \\
\left.-\bar{g}(2) A(q, r)_{t}\right) .
\end{array}
$$

In this step we prove that $G^{6}(q, r)^{n}$ satisfies (5.107). A simple calculation shows that [recall the notation $\bar{g}(2)_{n}$ of $(2.8)$ ]

$$
G^{6}(q, r)_{t}^{n}=\frac{1}{\Delta_{n}^{1 / 4}} \int_{0}^{t}\left(\frac{\bar{g}(2)_{n}}{k_{n}}-\bar{g}(2)\right) d A(q, r)_{s}+v(q, r)_{t}^{n},
$$

where because of (2.10) the remainder term $v(q, r)_{t}^{n}$ satisfies with $A^{\prime}(q, r)$ being the variation process of $A(q, r)$,

$$
\left|v(q, r)_{t}^{n}\right| \leq \frac{K}{\Delta_{n}^{1 / 4}}\left(A^{\prime}(q, r)_{u_{n}}+\left(A^{\prime}(q, r)_{t}-A^{\prime}(q, r)_{t-2 u_{n}}\right)\right) .
$$

In the continuous Case $\mathrm{C}$, we have $A^{\prime}(q, r)_{s+u_{n}}-A^{\prime}(q, r)_{s} \leq K u_{n}$, hence $\sup _{s \leq t}\left|v(q, r)_{s}^{n}\right| \leq K \Delta_{n}^{1 / 4}$. In the discontinuous Case D we only have $\mathbb{E}\left(A^{\prime}(q\right.$, $\left.r)_{s+u_{n}}-A^{\prime}(q, r)_{s}\right) \leq K u_{n}$ so that $v(q, r)_{t}^{n} \stackrel{\mathbb{P}}{\longrightarrow} 0$ as $n \rightarrow \infty$. Then if we apply (2.10) we obtain (5.107) for $l=6$.

Step 6. It remains to prove (5.107) for $l=5$. For this we use (5.4) again and Itô's formula to get, with $Y_{t}^{n, i}=\int_{i \Delta_{n}}^{t} g_{n}\left(s-i \Delta_{n}\right) d Y_{s}$ for any semimartingale $Y$ and for $t \geq i \Delta_{n}$

$$
\begin{aligned}
\left(\bar{X}_{i}^{n}\right)^{2} & -\int_{i \Delta_{n}}^{i \Delta_{n}+u_{n}} g_{n}\left(s-i \Delta_{n}\right)^{2} d[X, X]_{s} \\
= & 2 \int_{i \Delta_{n}}^{i \Delta_{n}+u_{n}} X_{s}^{n, i} g_{n}\left(s-i \Delta_{n}\right)\left(b_{s}^{q} d s+\sigma_{s} d W_{s}\right) \\
& +2 \int_{i \Delta_{n}}^{i \Delta_{n}+u_{n}} X_{s-}^{n, i} d M_{s}^{q} \\
& +2 \int_{i \Delta_{n}}^{i \Delta_{n}+u_{n}} \int_{\{\gamma(z)>1 / q\}} X_{s-}^{n, i} g_{n}\left(s-i \Delta_{n}\right) \delta(s, z) \underline{\mu}(d s, d z)
\end{aligned}
$$

and a similar expression with $\left(X, b^{q}, \sigma, \delta\right)$ substituted with $(X(q, r), b(q, r)$, $\sigma(q, r), \delta(q))$, so the second term on the right-hand side above vanishes in this case [remember the last part of (5.105)]. Therefore,

$$
\vartheta(q, r)_{i}^{n}=\frac{2}{k_{n} \Delta_{n}^{1 / 4}} \sum_{j=1}^{6} \eta(q, r, j)_{i}^{n}
$$


where, using (5.80) and with the notation $I(n, i)=\left(i \Delta_{n}, i \Delta_{n}+u_{n}\right]$, we have

$$
\begin{aligned}
& \eta(q, r, 1)_{i}^{n}=\int_{I(n, i)} X^{\prime}(q, r)_{s}^{n, i} g_{n}\left(s-i \Delta_{n}\right) d s\left(b_{s}+\int_{\{|\delta(s, z)|>1\}} \delta(s, z) \lambda(d z)\right), \\
& \eta(q, r, 2)_{i}^{n}=\int_{I(n, i)} X(q, r)_{s}^{n, i} g_{n}\left(s-i \Delta_{n}\right)\left(b_{s}^{q}-b(q, r)_{s}\right) d s, \\
& \eta(q, r, 3)_{i}^{n}=\int_{I(n, i)} X^{\prime}(q, r)_{s}^{n, i} g_{n}\left(s-i \Delta_{n}\right) \sigma_{s} d W_{s}, \\
& \eta(q, r, 4)_{i}^{n}=\int_{I(n, i)} X(q, r)_{s}^{n, i} g_{n}\left(s-i \Delta_{n}\right)\left(\sigma_{s}-\sigma(q, r)_{s}\right) d W_{s}, \\
& \eta(q, r, 5)_{i}^{n}=\int_{I(n, i)} X_{s-}^{n, i} g_{n}\left(s-i \Delta_{n}\right) d M_{s}^{q}, \\
& \eta(q, r, 6)_{i}^{n}=\int_{I(n, i)} \int_{\gamma(z)>1 / q} X^{\prime}(q, r)_{s-}^{n, i} g_{n}\left(s-i \Delta_{n}\right) \delta(s, z)(\underline{\mu}-\underline{\nu})(d s, d z) .
\end{aligned}
$$

Therefore, since $\eta(q, r, j)_{i}^{n}$ for $j=3,4,5,6$ are martingale increments, (5.107) for $l=5$ will follow if we prove that for all $t>0$, and as $m \rightarrow \infty$,

$$
\begin{aligned}
j & =1,2 \\
& \Rightarrow \quad \lim _{q \rightarrow \infty} \limsup _{r \rightarrow \infty} \limsup _{n \rightarrow \infty} \Delta_{n}^{1 / 4} \mathbb{E}\left(\sum_{i=0}^{\left[t / \Delta_{n}\right]-k_{n}}\left|\eta(q, r, j)_{i}^{n}\right|\right) \rightarrow 0, \\
j & =3,4,5,6 \\
& \Rightarrow \quad \lim _{q \rightarrow \infty} \limsup _{r \rightarrow \infty} \limsup _{n \rightarrow \infty} \Delta_{n}^{1 / 2} \mathbb{E}\left(\sum_{i=0}^{\left[t / \Delta_{n}\right]-k_{n}}\left|\eta(q, r, j)_{i}^{n}\right|^{2}\right) \rightarrow 0 .
\end{aligned}
$$

Then, standard estimates yield for $s \in I(n, i)$ and $p \geq 2\left[\right.$ recall $\left|b_{t}^{q}\right|+$ $\left.\left|b(q, r)_{t}\right| \leq K q\right]$

$$
\begin{aligned}
\mathbb{E}\left(\sup _{t \leq s}\left|X^{\prime}(q, r)_{t}^{n, i}\right|^{2}\right) & \leq K\left(\varepsilon(q, r)_{i}^{n}+\Delta_{n}^{1 / 2} \varepsilon_{q}\right), \\
\mathbb{E}\left(\sup _{t \leq s}\left|X(q, r)_{t}^{n, i}\right|^{p}\right) & \leq K_{p}\left(q^{p} \Delta_{n}^{p / 2}+\Delta_{n}^{1 / 2}\right), \\
\mathbb{E}\left(\sup _{t \leq s}\left|X_{t}^{n, i}\right|^{2}\right) & \leq K \Delta_{n}^{1 / 2}
\end{aligned}
$$

and it follows that, since $\left|g_{n}\right| \leq K$ and $\varepsilon(q, r)_{i}^{n} \leq K$ and $\varepsilon_{q} \leq K$ and

$$
\int_{\{|\delta(s, z)|>1\}}|\delta(s, z)| \lambda(d z) \leq \int \gamma(z)^{2} \lambda(d z)<\infty,
$$




$$
\begin{aligned}
& j=1,2 \Rightarrow \mathbb{E}\left(\left|\eta(q, r, j)_{i}^{n}\right|\right) \leq K \Delta_{n}^{1 / 2}\left(q \sqrt{\varepsilon(q, r)_{i}^{n}}+\Delta_{n}^{1 / 4} \sqrt{\varepsilon_{q}}\right) \\
& j=3,4,5,6 \Rightarrow \mathbb{E}\left(\left|\eta(q, r, j)_{i}^{n}\right|^{2}\right) \leq K \Delta_{n}^{1 / 2}\left(q^{2} \Delta_{n}^{3 / 4}+\varepsilon(q, r)_{i}^{n}+\Delta_{n}^{1 / 2} \varepsilon_{q}\right) .
\end{aligned}
$$

By Hölder's inequality,

$$
\left(\Delta_{n}^{3 / 4} \sum_{i=0}^{\left[t / \Delta_{n}\right]-k_{n}} \sqrt{\varepsilon(q, r)_{i}^{n}}\right)^{2} \leq \Delta_{n}^{1 / 2} \sum_{i=0}^{\left[t / \Delta_{n}\right]-k_{n}} \varepsilon(q, r)_{i}^{n} \leq K \varepsilon(q, r)_{t} .
$$

Since $\varepsilon(q, r) \rightarrow 0$ as $r \rightarrow \infty$, for each $q$, whereas $\varepsilon_{q} \rightarrow 0$ as $q \rightarrow \infty$. Therefore we readily obtain (5.109) and (5.110), and the proof is finished.

\section{REFERENCES}

[1] Aït-Sahalia, Y., Mykland, P. A. and Zhang, L. (2005). How often to sample a continuous-time process in the presence of market microstructure noise. Review of Financial Studies 18 351-416.

[2] Aït-Sahalia, Y. and Jacod, J. (2009). Estimating the degree of activity of jumps in high frequency data. Ann. Statist. 37 2202-2244. MR2543690

[3] Aït Sahalia, Y. and Jacod, J. (2009). Is Brownian motion necessary to model high frequency data? Ann. Statist. To appear.

[4] Aït Sahalia, Y. and Jacod, J. (2009). Testing for jumps in a discretely observed process. Ann. Statist. 37 184-222. MR2488349

[5] Bandi, F. M. and Russell, J. R. (2006). Separating microstructure noise from volatility. Journal of Financial Economics 79 655-692.

[6] Barndorff-Nielsen, O. E., Graversen, S. E., Jacod, J., Podolskij, M. and Shephard, N. (2006). A central limit theorem for realised power and bipower variations of continuous semimartingales. In From Stochastic Calculus to Mathematical Finance. Festschrift in Honour of A. N. Shiryaev (Y. Kabanov, R. Liptser and J. Stoyanov, eds.) 33-68. Springer, Heidelberg. MR2233534

[7] Barndorff-Nielsen, O. E., Hansen, P. R., Lunde, A. and Shephard, N. (2006). Designing realised kernels to measure the ex-post variation of equity prices in the presence of noise. Econometrica 76 1481-1536. MR2468558

[8] Barndorff-Nielsen, O. E. and Shephard, N. (2002). Econometric analysis of realised volatility and its use in estimating stochastic volatility models. J. $R$. Stat. Soc. Ser. B Stat. Methodol. 64 253-280. MR1904704

[9] Barndorff-Nielsen, O. E. and Shephard, N. (2004). Power and bipower variation with stochastic volatility and jumps (with discussion). Journal of Financial Econometrics 2 1-48.

[10] Cont, R. and Mancini, C. (2009). Nonparametric tests for analysing the fine structure of price fluctuations. Working paper.

[11] Gloter, A. and Jacod, J. (2001). Diffusions with measurement errors. II-Optimal estimators. ESAIM Probab. Statist. 5 243-260. MR1875673

[12] Ibragimov, I. A. and Has'minski, R. Z. (1981). Statistical Estimation: Asymptotic Theory. Springer, Berlin. MR0620321

[13] JACOD, J. (2008). Asymptotic properties of realized power variations and related functionals of semimartingales. Stochastic Process. Appl. 118 517-559. MR2394762 
[14] Jacod, J., Li, Y., Mykland, P., Podolskij, M. and Vetter, M. (2009). Microstructure noise in the continuous case: The pre-averaging approach. Stochastic Process. Appl. 119 2249-2276. MR2531091

[15] Jacod, J. and Protter, P. (1998). Asymptotic error distributions for the Euler method for stochastic differential equations. Ann. Probab. 26 267-307. MR1617049

[16] Jacod, J. and Shiryaev, A. N. (2003). Limit Theorems for Stochastic Processes, 2nd ed. Springer, Berlin. MR1943877

[17] Li, Y. and Mykland, P. (2007). Are volatility estimators robust with respect to modeling assumptions? Bernoulli 13 601-622. MR2348742

[18] PodolskiJ, M. and Vetter, M. (2009). Bipower-type estimation in a noisy diffusion setting. Stochastic Process. Appl. 119 2803-2831. MR2554029

[19] Podolskij, M. and Vetter, M. (2009). Estimation of volatility functionals in the simultaneous presence of microstructure noise and jumps. Bernoulli 15 634-658. MR2555193

[20] Tauchen, G. and Todorov, V. (2009). Activity signature functions with application for high-frequency data analysis. J. Econometrics 154 125-138.

[21] ZhANG, L. (2006). Efficient estimation of volatility using noisy observations. Bernoulli 12 1019-1043. MR2274854

[22] Zhang, L., Mykland, P. A. and Aït-Sahalia, Y. (2005). A tale of two time scales: Determining integrated volatility with noisy high-frequency data. $J$. Amer. Statist. Assoc. 100 1394-1411. MR2236450

J. JACOD

Institut de Mathématiques de Jussieu

UPMC (Université PARIs-6)

175 RUE DU CheVAleret

75013 PARIS

France

E-MAIL: jean.jacod@upmc.fr

\author{
M. Podolskij \\ ETH ZÜRICH \\ Department of Mathematics \\ 8092 ZÜRICH \\ SWITZERLAND \\ E-MAIL: mark.podolskij@math.ethz.ch
}

M. VetTeR

Ruhr-Universität Bochum

FAKUltät FÜr Mathematik

UNIVERSITÄTSSTR. 150

44780 BOCHUM

Germany

E-MAIL: mathias.vetter@rub.de 